\title{
On Line Spectrophotometric Measurement of Uranium and Nitrate in $H$ Canyon
}

\section{R.J. Lascola, R.R. Livingston, M.A. Sanders, J.E. McCarty, and G.A. Cooper}

\author{
Unclassified \\ Does Not Contain Unclassified Controlled Nuclear Information (UCNI)
}

July 31,2002

Westinghouse Savannah River Company Savannah River Site Aiken, SC 29808 
WSRC-TR-2002-00334

Revision 0

$\underline{\text { Keywords: }}$

Uranium, Spectroscopy, Chemometrics, Process Analysis

Classification: $U$

$\underline{\text { P.E. Filpus-Luyckx, ADS/SRTC }}$

Authorized Derivative Classifier

\section{On Line Spectrophotometric Measurement of Uranium and Nitrate in $\mathrm{H}$ Canyon}

R.J. Lascola, R.R. Livingston, M.A. Sanders, J.E. McCarty, and G.A. Cooper Publication Date: July 31, 2002

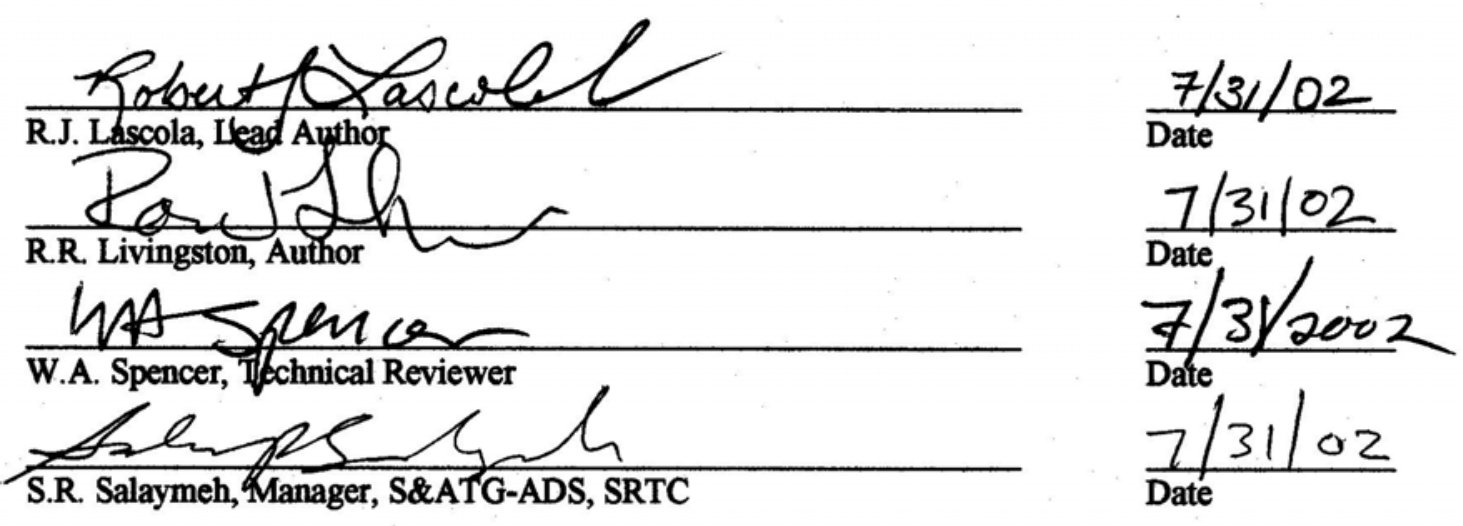

Westinghouse Savannah River Company Savannah River Site Aiken, SC 29808 
This document was prepared in conjunction with work accomplished under Contract No. DE-AC09-96SR18500 with the U. S. Department of Energy.

\section{DISCLAIMER}

This report was prepared as an account of work sponsored by an agency of the United States Government. Neither the United States Government nor any agency thereof, nor any of their employees, makes any warranty, express or implied, or assumes any legal liability or responsibility for the accuracy, completeness, or usefulness of any information, apparatus, product or process disclosed, or represents that its use would not infringe privately owned rights. Reference herein to any specific commercial product, process or service by trade name, trademark, manufacturer, or otherwise does not necessarily constitute or imply its endorsement, recommendation, or favoring by the United States Government or any agency thereof. The views and opinions of authors expressed herein do not necessarily state or reflect those of the United States Government or any agency thereof.

This report has been reproduced directly from the best available copy.

Available for sale to the public, in paper, from: U.S. Department of Commerce, National Technical Information Service, 5285 Port Royal Road, Springfield, VA 22161, phone: (800) 553-6847, fax: (703) 605-6900

email: orders@ntis.fedworld.gov

online ordering: http://www.ntis.gov/help/index.asp

Available electronically at http://www.osti.gov/bridge

Available for a processing fee to U.S. Department of Energy and its contractors, in paper, from: U.S. Department of Energy, Office of Scientific and Technical Information, P.O. Box 62, Oak Ridge, TN 37831-0062,

phone: (865)576-8401,

fax: (865)576-5728

email: $\underline{\text { reports@ adonis.osti.gov }}$ 


\begin{abstract}
Successful and timely completion of the Highly Enriched Uranium Blend Down Project requires an aggressive processing schedule. One of the requirements for meeting the schedule is the use of on-line instrumentation to eliminate the delays associated with the analysis of samples extracted from process tanks. The Analytical Development Section of the Savannah River Technology Center has developed on-line spectrophotometers to measure uranium and nitric acid concentrations in tanks in H Canyon. Development areas addressed in this report include air-lift samplers, instrument control and analysis software, and diode array spectrophotometers. A total of nine tanks will be analyzed using two spectrophotometers. The acquired spectra are interpreted using partial leastsquares models which are valid for solutions with uranium concentrations up to $11 \mathrm{~g} / \mathrm{L}$ and nitrate concentrations as high as $6 \mathrm{M}$. Concentration-dependent measurement uncertainities $(2 \sigma)$ are less than $0.30 \mathrm{~g} / \mathrm{L}$ for uranium and $0.32 \mathrm{M}$ for nitrate, and are comparable to the uncertainties of available diode array spectroscopy methods at the Analytical Laboratory (AL) facility. The models incorporate corrections for the spectral effects of $\mathrm{Fe}(<3 \mathrm{~g} / \mathrm{L})$ and $\mathrm{Hg}(<1 \mathrm{~g} / \mathrm{L})$, which are expected to be present in some process streams. Analyses with the on-line instrumentation can be performed in less than one minute, a large time savings compared to the many hours required for sampling, transport, and analysis of grab samples by AL. Other benefits of the on-line instrumentation include the reduction of radiological exposure to personnel and cost savings associated with fewer demands on AL and faster processing rates.
\end{abstract}




\section{TABLE OF CONTENTS}

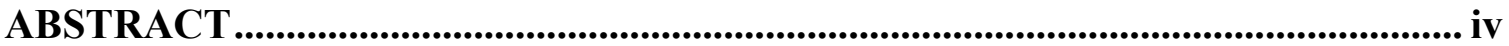

TABLE OF CONTENTS ........................................................................................

LIST OF FIGURES …............................................................................................ vi

LIST OF TABLES

1. SUMMARY ........................................................................................................................ 1

2. INTRODUCTION.................................................................................................................. 1

2.1 Measurement Principle............................................................................................................. 1

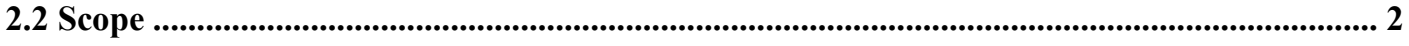

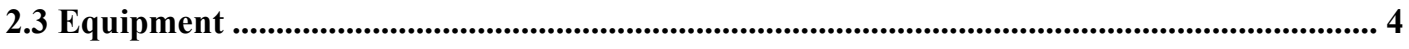

3. SAMPLER STUDIES................................................................................................... 7

3.1. Experimental details ................................................................................................................ 9

3.2. Sampler interface: single versus double needle ............................................................................ 11

3.3. Influence of operating conditions on sampler performance ................................................... 13

4. DATA ACQUISITION PROGRAMS......................................................................... 19

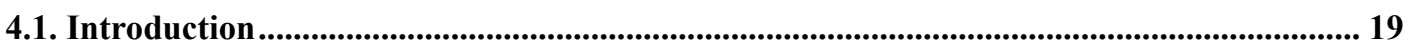

4.2. Program Operation ......................................................................................................................... 20

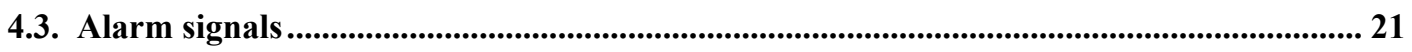

4.4. Data files and settings ............................................................................................................................... 25

5. PREDICTION MODELS................................................................................................ 31

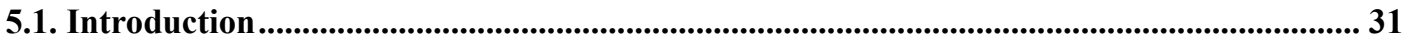

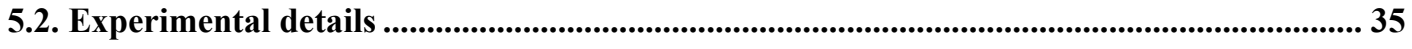

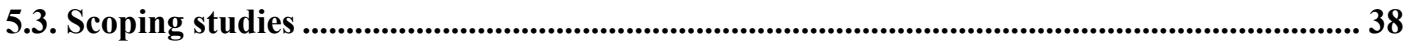

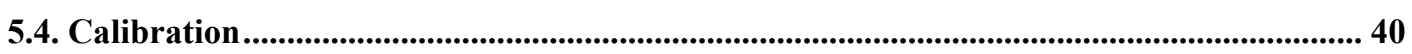

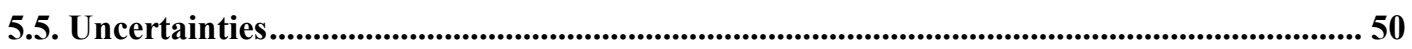

6. CONCLUSIONS ……...................................................................................................... 55

7. ACKNOWLEDGEMENTS ........................................................................................ 55

8. REFERENCES............................................................................................................ 56

APPENDIX 1 - Onlinez.exe Details ..................................................................................... 57

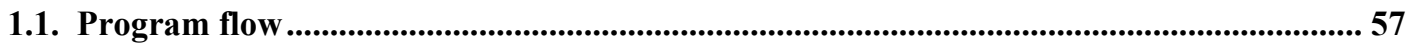


1.2. Functional Modules..

1.3. Visual Basic Modules and Forms contained in Onlinez program

APPENDIX 2 - Sample Uncertainty Calculation

\section{LIST OF FIGURES}

Figure 1. Schematic diagram of spectrophotometer system.

Figure 2. Schematic diagrams of air-lift sampler and fiber optic flow cell. 8

Figure 3. Schematic diagram of sampler mock-up. 10

Figure 4. Sampler/flow cell components. 12

Figure 5. Effects of air jet pressure on sample throughput. 14

Figure 6. Effects of air bleed rate on sample throughput. 14

Figure 7. Sampler performance with 4M sodium nitrate in process tank. 15

Figure 8. Sampler performance in H Canyon. 16

Figure 9. Sample exchange rate for flushing of $4 \mathrm{M}$ sodium nitrate with $\begin{array}{ll}\text { water. } & 17\end{array}$

Figure 10. Startup screens for data acquisition program Onlinez.exe. 21

Figure 11. Display during operation of Onlinez.exe. 22

Figure 12. Schedule display window (example). 26

Figure 13. Options display window (example). 27

Figure 14. UV-visible absorption spectra of uranyl nitrate. 32

Figure 15. Effect of temperature on uranyl nitrate spectroscopy. 33

Figure 16. Spectra of typical interferents. $\quad 33$

Figure 17. Distribution of calibration and validation set concentrations. 37

Figure 18. Effects of interferents on uranyl nitrate spectroscopy. 39

Figure 19. Temperature dependence of prediction errors for models created at $20^{\circ} \mathrm{C}$.

Figure 20. Effects of interferent spectra on calibration spectra. 42

Figure 21. Typical uranium prediction error dependence on uranium and nitrate concentrations, temperature, and interferent concentration. 44

Figure 22. Dependence of uranium prediction uncertainty on uranium concentration for three models.

Figure 23. Effects of normalization by [U] on spectra. 46

Figure 24. Typical nitrate prediction error dependence on uranium and nitrate concentrations, temperature, and interferent concentration. $\quad 47$

Figure 25. Dependence of nitrate prediction uncertainty on nitrate concentration for three models. $\quad 47$

Figure 26. Comparison of standard cuvette and sampler uncertainties. 52

Figure 27. Influence of sampler operation on analysis accuracy. 53

Figure 28. Total propagated uncertainties $(1 \sigma)$ for uranium and nitrate. 54 


\section{LIST OF TABLES}

Table 1. Process tanks and expected concentrations. 3

Table 2. Impurities found or expected in Tank 12.3. 3

Table 3. Effects of interferents on uranium and nitrate predictions. 40

Table 4. Tank/spectrometer/model concordance. 41

Table 5. $\quad$ Representative PLS results for uranium model. 43

Table 6. Representative PLS results for nitrate model. 45

Table 7. Effects of interferents on uranium and nitrate predictions. 48

Table 8. Effects of aluminum on uranium and nitrate predictions. 49

Table 9. Calibration and validation results for models. 49

Table 10. $\quad$ Field validation results for Tanks 14.5, 16.8, and 17.5. 51

Table 11. Uncertainties $(\sigma)$ for each measurement point. 55 


\section{SUMMARY}

This report describes the on-line instrumentation developed by the Analytical Development Section of Savannah River Technology Center (SRTC) in support of Highly Enriched Uranium (HEU) Blend Down processing in $\mathrm{H}$ Canyon. The system provides measurements of uranium and nitrate concentrations for nine tanks located before and after the mixer-settler separation banks. An air-lift sampler/flow cell system attached to each tank provides access to the contents of each tank. Absorption spectra of the solutions are obtained with one of two diode-array spectrophotometers that are coupled to the flow cells by fiber optics. The spectra are analyzed using partial least-squares analysis models, and the results are reported to the H Canyon Distributed Control System. This instrument replaces a spectrophotometer system developed and installed in the late 1980 's ${ }^{1}$ which was eventually abandoned after a redefinition of the site's priorities. Recent development work at SRTC incorporated improvements to the samplers, spectrophotometers, control software, and analysis models. This report describes those technical improvements and serves as an operating, design, and performance guide for the system.

\section{INTRODUCTION}

\subsection{Measurement Principle}

The basis of diode array spectroscopy as an analytical technique is the determination of the concentration- and wavelength-dependent light absorption of an analyte. The absorption is governed by Beer's Law: ${ }^{2}$

$$
A(\lambda)=\Sigma_{i} \varepsilon_{i}(\lambda) b c_{i}
$$

where $A$ is the total light absorption (a dimensionless quantity), $\lambda$ is the wavelength of light, $\varepsilon_{i}(\lambda)$ is the wavelength-dependent extinction coefficient of species $i, b$ is the optical pathlength, and $c_{i}$ is the concentration. The summation includes all chemical species that are present in the solution. The absorption is also defined by the relation:

$$
A(\lambda)=-\log _{10}(\mathrm{~T}(\lambda))
$$

where $\mathrm{T}$ is the fractional transmittance of the solution, compared to a blank solution (containing no analyte). For this application, the important aspects of Beer's Law are the following:

- The total absorption will be sum of the absorptions of the individual components. For example, the absorption of light by the uranium-nitric acid solution is due to the sum of absorptions by a series of uranyl nitrates $\left(\mathrm{UO}_{2}{ }^{2+}, \mathrm{UO}_{2}\left(\mathrm{NO}_{3}\right)^{+}, \mathrm{UO}_{2}\left(\mathrm{NO}_{3}\right)_{2}\right)$. Also, interfering species such $\mathrm{Fe}^{3+}$ and $\mathrm{Hg}^{2+}$ affect the measurements by absorbing light at the same frequencies as the uranyl nitrates. Detector noise and other instrumental artifacts lead to deviations from Beer's Law for absorbances greater than $\sim 1.5$. 
- The wavelength-dependent nature of the absorbance of each species potentially allows the contribution of each component to be extracted from the total spectrum. Since, for example, $\mathrm{Fe}^{3+}$ and $\mathrm{Hg}^{2+}$ have different absorption spectra than the uranyl nitrates, one can compensate for the effects of these ions on the total spectrum so long as the total absorbance remains below $\sim 1.5$.

- Pathlength and concentration are inversely correlated. A smaller concentration will give the same response as a larger concentration if a correspondingly longer pathlength is used for the measurement. This relationship is the basis for the selection of the optical pathlength of the flow cell.

\subsection{Scope}

The scope of analyte concentrations and matrix conditions in which the system is designed to perform is listed in the Task Technical Plan for spectrophotometer development. Those quantities are reproduced in Tables 1 and 2. The total instrument consists of two spectrophotometers operating in parallel. This arrangement allows the monitoring of adjacent tanks in the process without violating common mode failure restrictions associated with the Double Contingency Analysis of H Canyon. Tanks 12.2 and 12.3 (1AF stream) are located immediately prior to the first set of mixer-settler banks, on the "hot" side of H Canyon. These tanks are expected to have the highest concentrations of potentially interfering species and the highest radiological dose rates. The remaining tanks are located on the "warm" side of H Canyon, either between the two sets of banks (16.8, 17.5, 18.1, and 18.7; 1CU and 1DF streams) or after the second set $(14.5,15.4$, and 17.4; $1 E U$ stream). The primary source of interferents at these tanks is pipe degradation, and the interferent concentrations are expected to be much lower than for Tanks 12.2 and 12.3. Dose rates will also be much lower. The spectrophotometer system is designed to measure uranium concentrations between $0-11 \mathrm{~g} / \mathrm{L}$ and nitrate concentrations between 0.05 and $6 \mathrm{M}^{*}$ "These ranges span the expected concentrations at the tanks which will be monitored. The system will also accommodate solution temperatures of $20-45{ }^{\circ} \mathrm{C}$, as measured at the flow cell. The temperature range does not represent the temperature of the solution in the tank. The solution temperature will equilibrate with the building temperature as the solution travels through the pipes from the tanks to the flow cells. Under these conditions, the system will measure uranium and nitrate concentrations with a concentration-dependent uncertainty between $0.10-0.30 \mathrm{~g} / \mathrm{L}(2 \sigma)$ for $U$ and $0.18-0.32 \mathrm{M}(2 \sigma)$ for $\mathrm{NO}_{3}{ }^{-}$. The uncertainty is proportional to the analyte concentration, so many measurements will have a lower uncertainty. The measurement uncertainties are fully discussed in Section 5.5.

A historical note concerning the scope of this application is in order. The instrument is not designed to measure waste streams in the HEU process. These streams - 1AW, 1DW, 1BP, HAW Bottoms, and LAW Bottoms - are commonly characterized by low U concentrations (0.1-100 mg/L), substantial iron concentrations (typically $1-4 \mathrm{~g} / \mathrm{L}$, but up to

\footnotetext{
* The only source of nitrate in the HEU process comes from nitric acid. In this report, the terms "nitrate" and "acid" will be used interchangeably.
} 
Table 1. Process tanks and expected concentrations.

\begin{tabular}{|c|c|c|c|}
\hline Tank & Spectrometer & $\mathrm{U}(\mathrm{g} / \mathrm{L})$ & Nitrate $(\mathrm{M})$ \\
\hline 12.2 & 1 & $4.2-5.2$ & $1.6-2.2$ \\
\hline 12.3 & 2 & 66 & “6 \\
\hline $14.2^{\mathrm{a}}$ & 2 & 3.5 & 0.35 \\
\hline 14.5 & 1 & 7.8 & 0.10 \\
\hline 15.4 & 1 & 66 & 66 \\
\hline $15.7^{\mathrm{a}}$ & 1 & 6 & 66 \\
\hline 16.8 & 1 & 5.7 & 4.3 \\
\hline 17.4 & 2 & 7.8 & 0.10 \\
\hline 17.5 & 1 & 3.5 & 0.35 \\
\hline 18.1 & 2 & “6 & “6 \\
\hline 18.7 & 2 & 5.7 & 4.3 \\
\hline
\end{tabular}

$\mathbf{a}-$ These tanks are currently monitored with colorimeters. Their incorporation into the spectrophotometer system has been deferred.

Table 2. Impurities found or expected in Tank 12.3.

\begin{tabular}{|c|c|c|}
\hline Impurity & ${\text { Concentration }(\mathbf{g} / \mathbf{L})^{\mathbf{a}}}{\text { Flowsheet }(\mathbf{g} / \mathbf{L})^{\mathbf{b}}}^{\mathbf{2}}$ & 35 \\
\hline $\mathrm{Al}$ & 25 & --- \\
\hline $\mathrm{Cr}$ & 0.012 & --- \\
\hline $\mathrm{Cu}$ & 0.027 & $1.3^{\mathbf{c}}$ \\
\hline $\mathrm{Fe}$ & 0.19 & 0.77 \\
\hline $\mathrm{Hg}$ & --- & --- \\
\hline $\mathrm{Mg}$ & 0.12 & --- \\
\hline $\mathrm{Mn}$ & 0.008 & 4.6 \\
\hline $\mathrm{Na}$ & --- & --- \\
\hline $\mathrm{Ni}$ & 0.12 & --- \\
\hline $\mathrm{Pb}$ & 0.038 & --- \\
\hline $\mathrm{Zn}$ & 0.015 & \\
\hline
\end{tabular}

a-Concentrations determined by ICP analysis of 5 aliquots taken on three days. Results are maximum values, rounded up. $\mathbf{b}$-Concentrations based on flowsheet calculations (e-mail, C. Pickett to M.

Bronikowski, 5/15/01). $\mathbf{c}-$ Sum of +2 and +3 oxidation states. $1 \mathrm{~g} / \mathrm{L}$ approximately equals $1300 \mathrm{ppm}$.

The contents of Tanks 12.2 and 12.3 are expected to be similar.

$30 \mathrm{~g} / \mathrm{L}$ in LAW bottoms), and potentially significant concentrations of other actinides. The combination of high Fe and low $U$ concentrations makes $U$ analysis by diode array spectroscopy nearly impossible. Ferrous $\left(\mathrm{Fe}^{2+}\right)$, ferric $\left(\mathrm{Fe}^{3+}\right)$, and uranyl nitrate ions absorb light at similar wavelengths, and the Fe absorptions are stronger at these concentrations. Low-assay $U$ analysis would be possible by diode array spectroscopy if long optical pathlengths were used. However, $\mathrm{Fe}$ at the concentrations described above would absorb all light at the relevant wavelengths and mask any $U$ response.

There are several techniques which could remove Fe (and other potential interferents such as $\mathrm{Hg}, \mathrm{Ni}, \mathrm{Cr}$, etc.) from the solutions, and also provide for enhanced sensitivity to $\mathrm{U}$ by the formation of a highly colored complex. ${ }^{3,4}$ However, these solutions would require automated chemistry to separate the elements on a chromatographic column and 
form the colored complex. The additional maintenance and uncertainty associated with that equipment made that option unfeasible for those tanks, and off-line techniques were found to be more suitable. Still, if the need and commitment for an on-line analysis reemerges, these techniques may be revisited.

The spectrophotometer system will be used for analysis of the 1AF stream (Tanks 12.2 and 12.3), where significant $\mathrm{Fe}$ and $\mathrm{Hg}$ concentrations are expected. In this case, the uranium concentration is also high $(\sim 5 \mathrm{~g} / \mathrm{L})$, and its absorption is of similar magnitude to the interferent absorption. Thus, there is enough signal to permit uranium and nitrate analysis in these tanks.

\subsection{Equipment}

A schematic diagram describing each spectrophotometer is shown in Figure 1. Light from a xenon (Xe) arc lamp is coupled into a fiber optic and carried to an optical multiplexer. Under computer control, the multiplexer directs the light to one of a series of fiber optics which carry the light to either a reference or standard, or to a flow cell. The light interacts with the sample and is partially absorbed. The transmitted light is coupled into another fiber optic, which returns the light to the multiplexer. The light is routed to a

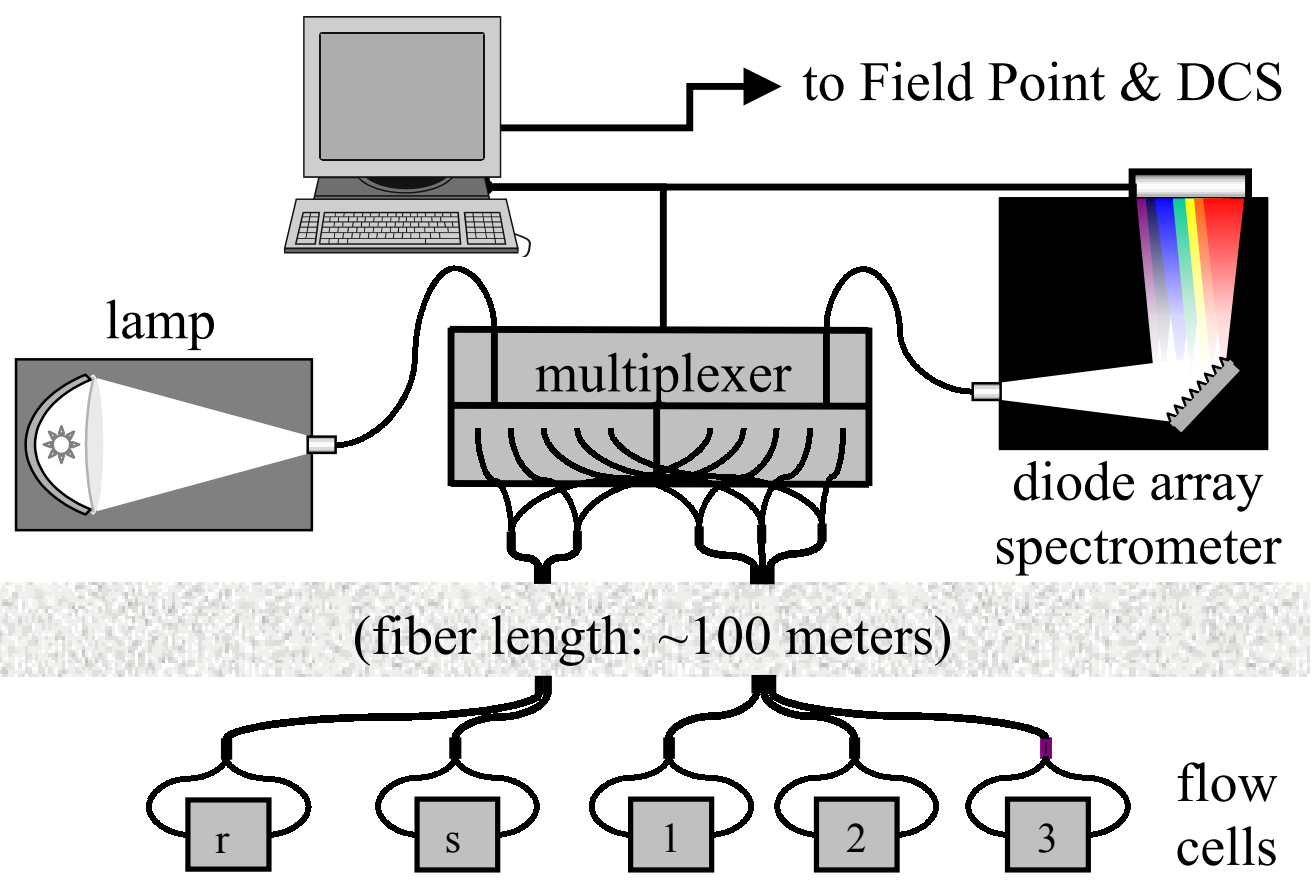

Figure 1. Schematic diagram of spectrophotometer system. 
spectrometer, where it is dispersed by wavelength using a holographic grating and focused onto a diode array. The computer reads the intensity of light incident on each diode, calculates a spectrum, performs signal averaging and data analysis, and transmits the resulting concentrations to a series of Field Point modules which serve as the interface between the instrument and the H-Canyon control room DCS. Equipment details and comments on their use are provided below. Note that the two spectrophotometers were installed approximately two years apart, and some aspects of the equipment may have superficial differences (they are, however, functionally equivalent).

The spectrometer design and equipment described below is incorporated in a H Canyon design package. That package also includes detailed specification for the interface between the Field Point modules and the DCS, which are not described here.

Lamps. Hamamatsu (Bridgewater, NJ; part no. L2174) 75 watt ozone-free Xe arc lamps are mounted in Oriel (Stratford, CT; part no. 66057) housings with a condenser (Oriel; part no. 66150), iris diaphragm (Oriel; part no. 6203), and SMA mount (Oriel; part no. 77800) for the fiber optics. The lamp is powered by a Hamamatsu power supply (part no. C2177-01). Xenon arc lamps provide the best combination of long life (average 3000 hours) and strong output in the near ultraviolet and visible regions of the spectrum where uranyl nitrate absorbs light. The Hamamatsu lamps are well suited for fiber optic spectroscopy applications by virtue of their particularly low noise and small spot size, which is more efficiently focused into a fiber.

Fiber optics. Fiber optics were purchased from Ceramoptec (Longmeadow, MA) or Polymicro Technologies (Phoenix, AZ). They are commonly characterized as UVenhanced multimode fibers with a step index, a 400 micron high $\mathrm{OH}$ core, 440 micron doped silica cladding, Kevlar strength members, and a polyimide jacket, with a numerical aperture of 0.22 . The fibers are terminated with stainless steel SMA 905 connectors, and epoxy is used to hold the fibers in place in the connectors. The fibers which connect the spectrophotometer on the fourth level to the samplers on the third level are in a duplex package, in which two fibers are contained in a secondary polyimide jacket. Fibers which will be exposed in the sample aisles have been removed from the secondary polyimide jacket and enclosed in a flexible stainless steel jacket manufactured by Precision Hose, Inc. (Stone Mountain, GA; part no. 5/32-PHI-SL-S). Seals at each end are made with epoxy and shrink wrap tubing. The stainless jacketing provides extra protection against crushing and bending of the fibers, as well as some resistance to acid. Fibers lengths from the spectrophotometers to the tanks are not greater than 120 meters.

Flow cells. Details of air-lift sampler operation, including a diagram of the flow cell, will be provided in Section 3. The flow cell which is attached to each sampler is constructed from stainless steel compression fittings and tubing which are welded to promote the solution flow. The optical region is defined by a "T" fitting at the bottom of the flow cell, where the solutions enters and exits at the top, and light is coupled at the sides with collimating lenses (Equitech International, New Ellenton, SC; part no. CL-UV-K6375). The lenses have 316L stainless steel bodies which accept compression fittings, and have internal Kalrez o-ring seals to prevent leakage around the fused silica lenses. The lenses 
press against internal shoulders on the " $T$ ", which define the optical path length. The nominal path length is $2.54 \mathrm{~cm}(1$ "); shorter lengths can be defined by modifying the " $T$ ". Fiber optics are coupled to the lenses with standard SMA fittings. The relative position of the SMA fiber to the collimating lens is established with a stainless steel locking nut on the SMA fitting. There also are two cylindrical shields which screw onto the outer protruding ends of the lenses and provide strain relief for the fibers.

Optical multiplexers. Multiplexers were provided by DiCon Fiberoptics (Richmond, CA; part no. GP700-4-2/1x12-400-SMA-B-Z). Each multiplexer contains two $1 \times 12$ switches constrained to operate synchronously. Internal fiber optics (supplied by SRTC to the vendor) match the fiber optic specifications described above. External fiber optics connect at SMA bulkhead adapters mounted on the rear panel. Initialization of the multiplexer and its positioning during data acquisition is controlled by the data acquisition program via a RS-232 interface. Handshaking protocols between the multiplexer and the computer provide sensitivity to multiplexer failure modes. Details of the computer control program are provided in Section 4.

Spectrometer. The diode array spectrometer is constructed at SRTC with commercially available components. The light dispersion and detection unit is a Zeiss MCS-501 UVNIR module with 1024 elements (SpectrAlliance, St. Louis, MO; part no. 81-654). Array signals are read and amplified with a Hamamatsu C4070 circuit board, and power is supplied from a Condor (Oxnard, CA; part no. MTLL-5W-A) power supply. The wavelength dispersion of the spectrometer is calibrated prior to data acquisition by measuring peak positions of the output of an Ocean Optics (Dunedin, FL; part no. HG-1) mercuryargon lamp. The spectrometer has no moving parts and is constructed of highly stable materials. Communication with the controlling computer is achieved with a custom Amphenol cable (Black Box, Pittsburgh, PA; part no. CBCC154940) and a 16-bit input/output card (National Instruments, Austin, TX; part no. 777422-01).

Computer. Standard rack-mounted industrial computers are used for each spectrophotometer. The computers must be able to communicate with the spectrometer, Field Point modules, and multiplexer, whether through existing ports or by the addition of cards. The computers must also be able to run the data acquisition program. The less capable of the two computers has a $266 \mathrm{MHz}$ processor with $32 \mathrm{MB}$ of memory and a $2 \mathrm{~GB}$ hard drive, and uses the Windows 95 operating system. The data acquisition program has been tested on both Windows 95 and 98. A Zip drive or CD writer allows archival of the large amount of data acquired during operation. Both computers are connected to a rackmounted flip-up LCD monitor/keyboard/mouse unit (ICP America, Vista, CA; part no. LKM-9265C) that accepts up to five inputs. The display can switched manually or automatically between each computer.

Field Point modules. Field Point modules (National Instruments) accept signals from the computers through an RS-232 or RS-485 cable and route the signals to individual analog or digital sensors. Each computer/spectrometer has its own bank of Field Point modules. The Field Point bank consists of a controlling unit (part no. 777517-01) and a series of analog (part no. 777518-200) and digital (part no. 777518-420) output units. 
The controlling unit reads a string from the computer which specifies a value and the address in the bank to which the value should be sent. The value is determined by the output range of the bank module (defined in configuration software run from the computer) and the value that is being written. In this application, the analog values represent uranium or nitrate concentrations, and the digital values represent alarm status. The analog output range is defined as 6-20 mA; the region from 3.5-6 mA is used by the DCS for diagnostics. There are two analog and two digital outputs for each measurement point. In addition, for each computer there is a digital watchdog output which serves as a general trouble alarm. This alarm is activated if there is a problem with the multiplexer or spectrometer. There is a second watchdog alarm administered by the Field Point controller which activates if a regular communication from the computer is not received.

Other components. The reference and standard cells are located in two junction boxes in the Warm Sample Aisle. The cuvettes (Spectrocell, Oreland, PA; part no. R-2010-T) are quartz, $1 \mathrm{~cm}$ pathlength, and are sealed with Teflon-lined caps. The cuvette holders are made of Lexan, and incorporate the same fiber optic collimating lenses as are used in the flow cells.

\section{SAMPLER STUDIES}

The air-lift samplers used to draw solution aliquots from the process tanks to the sample vials and flow cells have been described previously. ${ }^{1,6}$ A schematic diagram of the sampler is shown in Figure 2(a). An air jet creates a vacuum which lifts solution out of the tank. As the sample vial and flow cell are located 35 feet above the tank level, vacuum alone is not sufficient to raise the solution to the sampler box. Therefore, air is bled into the sample line to create slugs of a liquid-gas mixture which can be lifted to greater heights. The slugs fill the sample vial and the overflow enters the flow cell. In the cell (see Figure 2(b)), the solution enters through the inner of two concentric tubes, fills the optical region between the fiber optic couplers, and exits through the outer tube, eventually returning to the tank.

This design allows sample collection and analysis with a minimum of moving parts, which reduces the need for maintenance in a radioactive environment. Still, the design leads to several problems. The entrained air interferes with the spectroscopic measurement by scattering light in the flow cell, producing a baseline offset, and displacing liquid, which reduces the effective optical pathlength and consequently diminishes the absorption spectrum. Therefore, acceptable data can only be taken when bubbles are absent or below a certain level. That level can be defined by the intensity of light transmitted through the cell at a wavelength where the solution is not expected to absorb, and any loss of light can be attributed to the bubbles. Measurements below that level can be ignored (this technique is incorporated in the data acquisition program described below). Still, this level of control is purely passive, and does not address the situation in which the turbulence is too great to obtain good signals. 


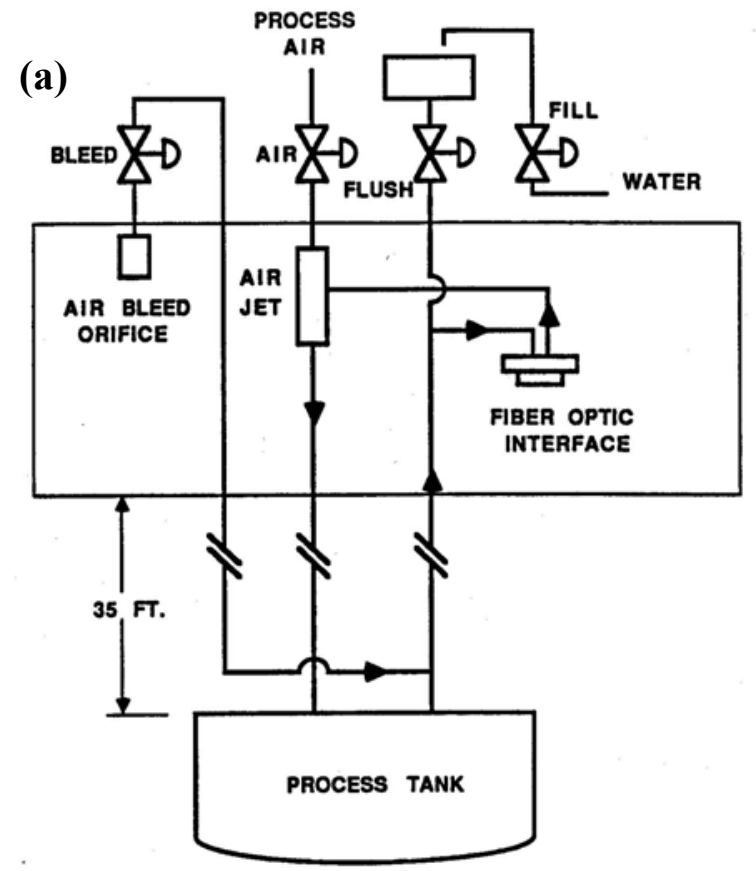

(b)

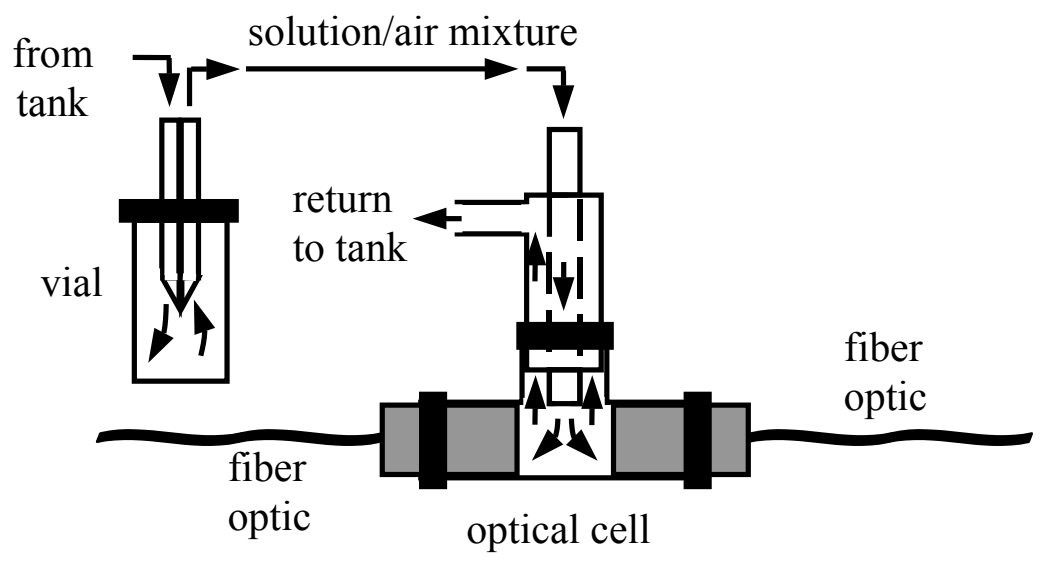

Figure 2. Schematic diagrams of air-lift sampler and fiber optic flow cell.

In addition, the air/liquid mixture promotes solution evaporation, which can lead to plugged lines, especially for solutions with high salt content. The salt can be redissolved with a process water flush, but such steps are time-consuming and do not improve the reliability of the system. In this application, several tanks (12.2 and 12.3) which will be monitored are located before the first mixer-settler banks, and thus have potentially high concentrations of metal ions (particularly aluminum). Anecdotal evidence suggests that salt buildup prevented the long term use of the previous spectrophotometer system to monitor these tanks. 
The installation of new flow cells to Tanks 12.2 and 12.3 also requires consideration of the radiological impact of maintaining a larger quantity of solution in the sampler box. At those points in the process, the stream contains radiologically significant quantities of transuranics. Sample aisle operator exposure, with the sampler box door open and shut, must be considered.

In light of these potential problems, SRTC studied several operational parameters to optimize the performance of the air-lift samplers:

- The effects of single- versus double-needle design for the sample vial interface.

- The interactions of air jet pressure, air bleed, and solution viscosity on sampling rate and light throughput in the flow cell.

- Anticipated worker exposure for the addition of a flow cell to the sampler box.

- Orientation of the flow cell in the sampler box, for hot sample aisle operation.

To conduct these studies, SRTC constructed a mock-up of the air-lift sampler, which is described below.

\subsection{Experimental details}

Equipment. The sampler mock-up was erected in the Thermal Fluids Laboratory of SRTC (786-A). The rigging bays of this facility are large enough so that the horizontal and vertical displacements of the H-Canyon sampler can be reproduced. A schematic of the mock-up is shown in Figure 3. Compared to the H-Canyon system, the mock-up includes several extra leak valves and air flow meters, which are attached to the sample and bleed lines to simulate leaks in these lines. An extra pressure gauge and flow rate meter allow quantification of the air jet pressure and bleed rate, respectively. The sample supply and air bleed lines are 3/8" steel tubing, and the sample return line is $3 / 4$ " steel tubing. These dimensions provide the same inner dimensions as the pipes in H-Canyon (1/8" and 1/2" Schedule 40 pipe, respectively). The Hanford connector fittings are replaced by Swagelock fittings. A demister is attached to the sample return line to allow air to escape the system. The tank is a 25 liter plastic carboy. An external recirculation pump attached to the tank allows circulation of the solution through a heat bath and allows easy addition of water or colored liquid to the tank. The sampler box is constructed of Lexan, but is otherwise identical to the sample boxes in H-Canyon. Flow cell performance is monitored with a spectrophotometer (functionally identical to the instrument installed in the field, except that a multiplexer was not used).

Solutions. Basic sampler operation and leak checking are conducted with water. Operation with a higher density solution is tested with a $4 \mathrm{M}$ sodium nitrate solution with a measured density of $1.208 \mathrm{~g} / \mathrm{mL}$. That density is greater than solution densities in First and Second Uranium Cycles and slightly less than the densities observed for Tanks 12.2 and $12.3(1.3 \mathrm{~g} / \mathrm{L})$. Fluorescein green is used to color solutions and provide an estimate of sample exchange times. 


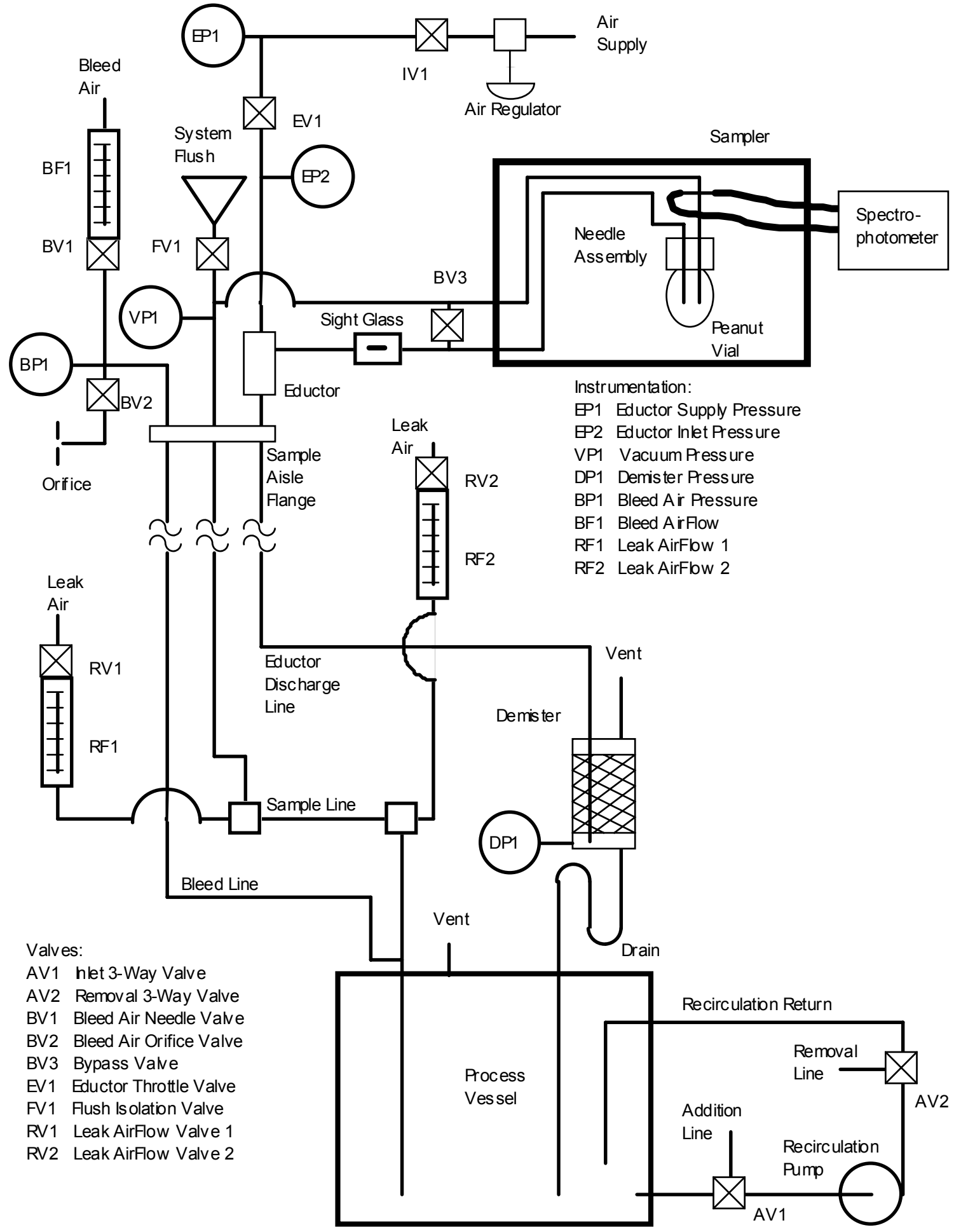

Figure 3. Schematic diagram of sampler mock-up. 


\subsection{Sampler interface: single versus double needle}

The historical design for the sample vial interface incorporates a double needle design, as shown in Figure 4(a), in which two 0.09" O.D. tubes are ground to a point. Solution flows into the sample vial through one tube and is educted out the second tube towards the flow cell. The vial is capped with two rubber septa which are penetrated by the needles when the vial is installed and reseal when the vial is removed. We compared the performance of the flow cell with the double needle arrangement versus a single-needle design, also shown in Figure 4(a), in which the two tubes are replaced with a single, larger tube $(0.25$ " O.D.) which is divided in half by a thin plate.

We observed several advantages to using the single needle design. First, as might be expected from the larger tube size, we measured a 2-3x greater flow rate with the single needle for a variety of air jet pressures and pinhole leak flow rates. For example, with jet pressure $\mathrm{P}_{\text {jet }}=110 \mathrm{psi}$ and leak flow rate $\mathrm{F}_{\text {leak }}=2.3 \mathrm{~L} / \mathrm{min}$, the single and double needle liquid exchange rates for water were $270 \mathrm{~mL} / \mathrm{min}$ and $80 \mathrm{~mL} / \mathrm{min}$, respectively. Both flow rates are adequate for timely solution collection. Second, the double needle penetration occasionally leaked during operation. Slight leaks were observed on about $1 / 3-$ $1 / 2$ of vial insertions, and in all cases, when vials were removed a small drop of solution was observed on top of the vial. We attribute the leakage to solution adhesion inside the smaller diameter of the double needles, subsequently drawn out by contact with the septa as they are pulled past. In contrast, the solution drains completely from the single needle. We did not observe any leaks with the single needle, either during operation or vial removal. Third, the double needle design presents a greater risk for plugging. Since the liquid slugs cascade intermittently through the system during operation, there is ample opportunity for air to dry droplets stuck inside the needles or at the tips. The smaller needles of the double needle design will plug more quickly than the single needle design.

We therefore recommended using the single needle design for all new samplers in $\mathrm{H}$ Canyon. This recommendation was accepted by NMMD design personnel, who have accordingly updated the system designs. Consequently, the experiments reported in this section emphasize the performance of the single needle design.

An exploded view of the flow cell assembly is shown in Figure 4(b). Parts are labeled as follows: (1), flow cell with collimating lenses; (2), single needle; (3), peanut vial and cap with septum; (4) and (5), peanut vial holder, bottom and top. 
(a)

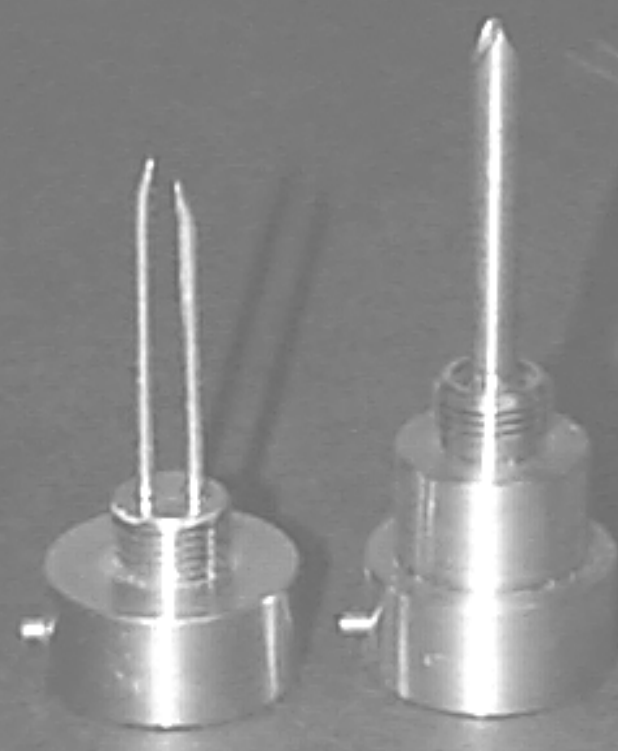

(b)

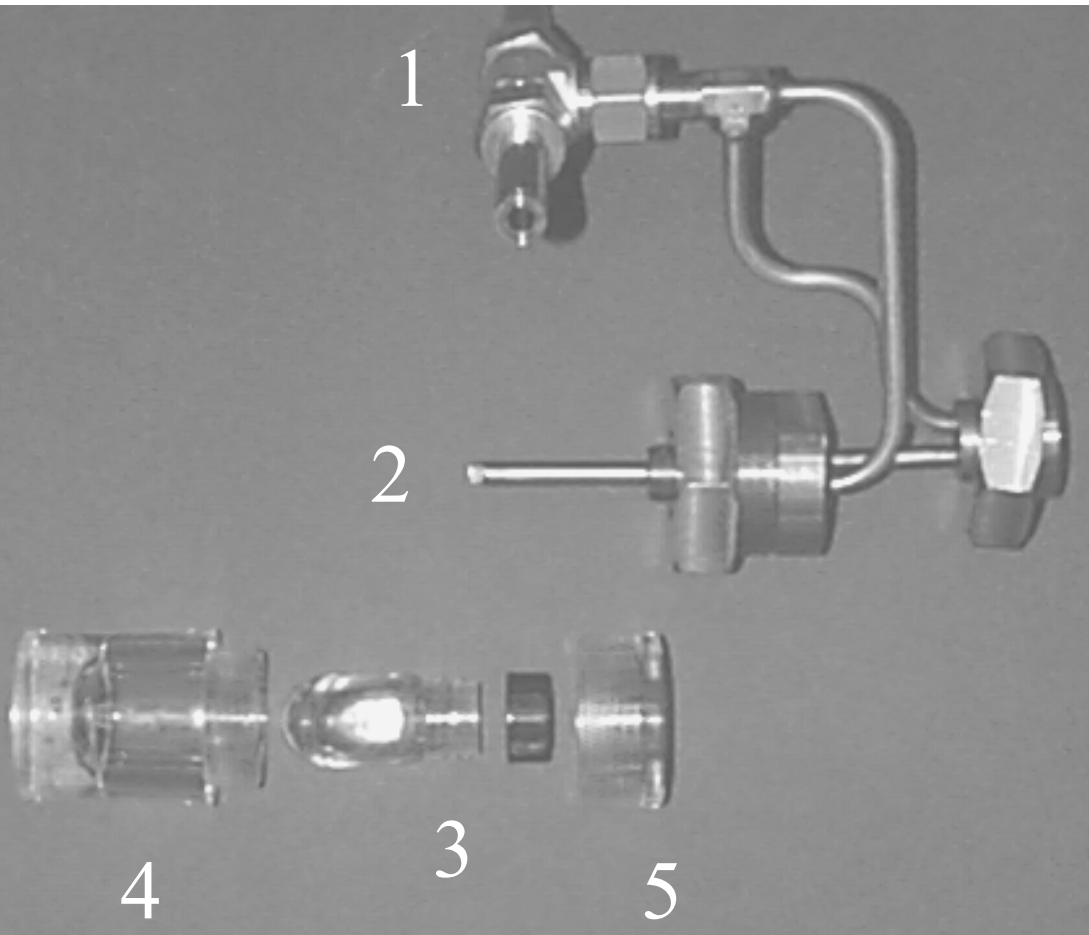

Figure 4. Sampler/flow cell components. 


\subsection{Influence of operating conditions on sampler performance}

As stated above, during sampler operation the flow of liquid into the sample vial and flow cell is not continuous. Instead, the mixed air/liquid sample arrives as slugs separated by air. Turbulence in the flow cell can arise from two situations - bubbles in the sample slug which have not yet dissipated, and air blown through the liquid before the arrival of the next slug. We have observed that the importance of these mechanisms is related to the density of the solution, the jet pressure, and the leak flow rate.

The effect of air jet pressure on a less dense solution (water) can be seen in Figure 5. For these time traces, the air bleed leak rate is held constant at $2.4 \mathrm{~L} / \mathrm{min}$, the jet pressure is changed at valve EV1 (see Figure 3), and the pressure is read at gauge EP1. In this case, less than the full amount of jet pressure is required to bring the solution to the flow cell liquid flow rates were relatively high for pressures ranging from 45-110 psi - and there is evidence that too much pressure can lead to extra turbulence in the flow cell. Features in the time trace correlate with observations of the flow in the sample vial as follows: Areas of complete intensity dropout (labeled (1)) have no solution flow through the vial but strong turbulence from large amounts of air entering the vial. The liquid levels in the vial are below the level of the needles, so no solution enters the flow cell. The intensity throughput for a completely dry flow cell would not be affected by air blowing through it, so the loss of intensity suggests that there is solution trapped in the flow cell. Just before a slug of solution appears in the vial (2), the air blowing suddenly subsides. As no liquid is being introduced, any changes in the flow cell throughput are due to changes in the solution already there. Specifically, the trapped solution is not being disturbed by the air flow. Eventually, an air/liquid slug cascades through the peanut vial (3), and the liquid level in the vial rises above the needle level until the slug is gone.

The air/liquid mix is also determined by the leak rate. Figure 6 shows the effect of changing the leak rate with a constant jet pressure (120 psi), again for water. The top trace here is consistent with the top trace in Figure 5, with a high jet pressure and leak rate. As the leak rate is reduced, the turbulence decreases, as does the liquid flow rate. Interestingly, the liquid flow rate does not immediately reduce to zero when the air bleed is completely closed. The simulated leak valves were closed during this test, so no air should have entered the system. As the flow would eventaully go to zero with the leak valves closed, there were no other leaks in the system. During the course of these tests we observed that the liquid flow rate responded slowly to changes in the air bleed rate (though much more quickly to changes in the jet pressure). The slow response could be due to the outgassing of dissolved air in the solution. We observed strong turbulence in the reservoir from the returning solution, and the circulated volume was small $(<10 \mathrm{~L})$, so it is likely that large amounts of air were dissolved in the liquid. Therefore, the true liquid flow rate under these conditions is probably lower than what we recorded. Also note that the correlation of turbulence to observed activity in the sample vial is similar to the situation described in the previous paragraph.

Introduction of a solution containing $4 \mathrm{M}$ sodium nitrate to the process tank significantly changed sampler operation, in that similar jet pressures and air bleed rates generated less 


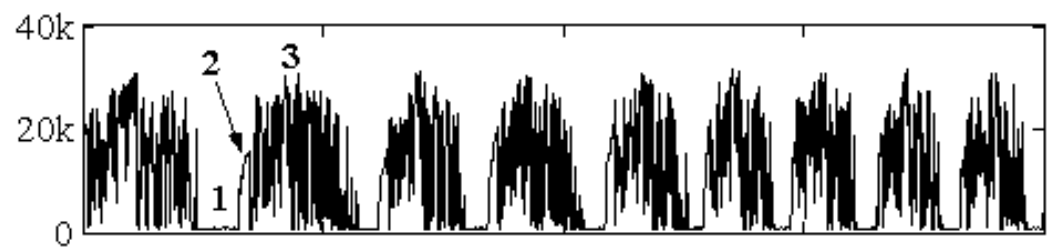

$\mathbf{P}_{\text {jet }}=$

120 psi.

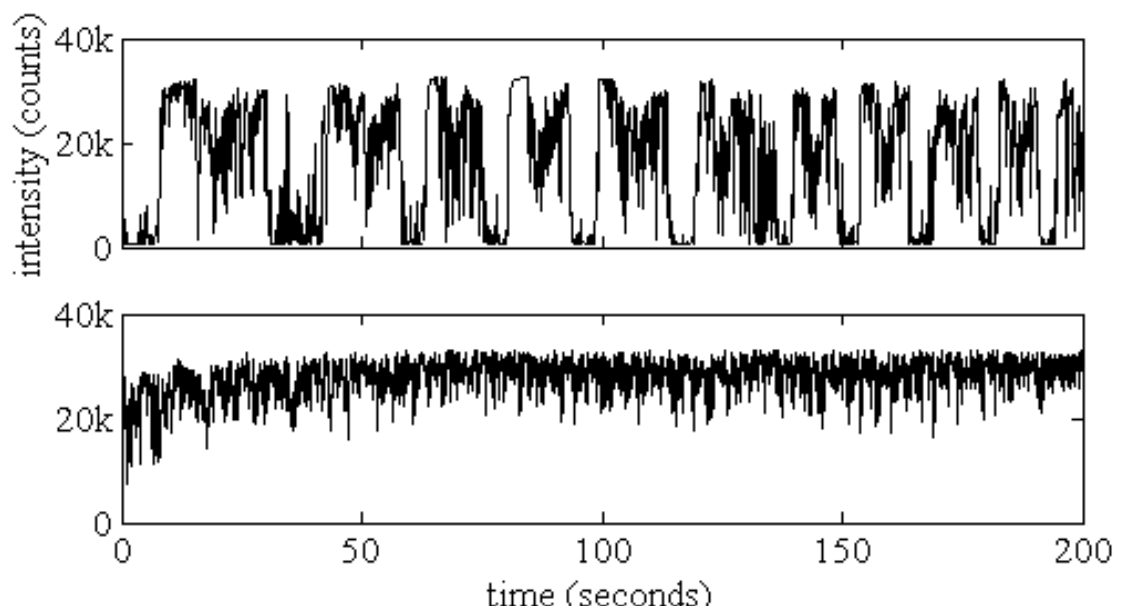

$\mathbf{P}_{\text {jet }}=$

45 psi.

$\mathbf{P}_{\text {jet }}=$

22 psi.

Figure 5. Effects of air jet pressure on sample throughput.

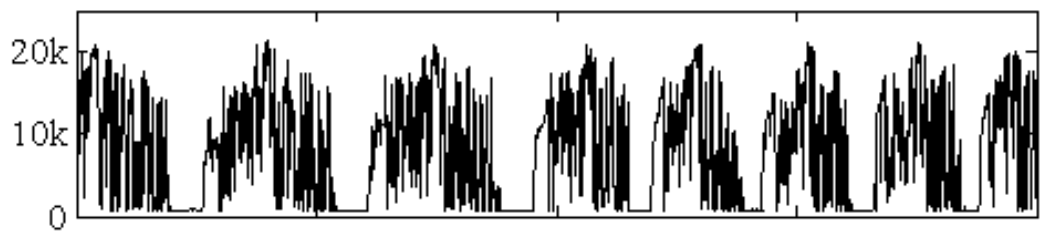

$\mathrm{F}_{\text {leak }}=$

$2.4 \mathrm{~L} / \mathrm{min}$.

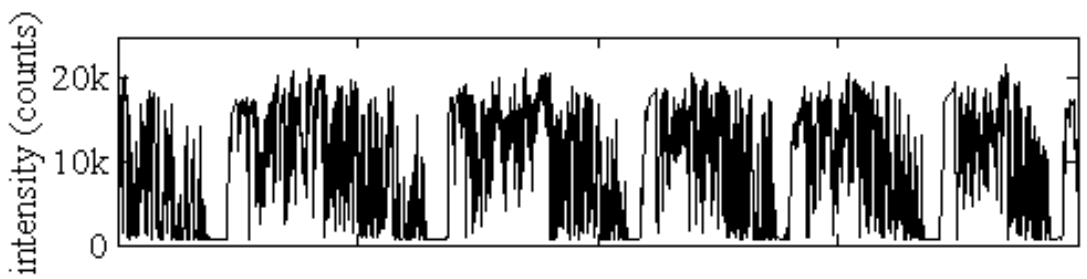

$\mathbf{F}_{\text {leak }}=$

$1.2 \mathrm{~L} / \mathrm{min}$.

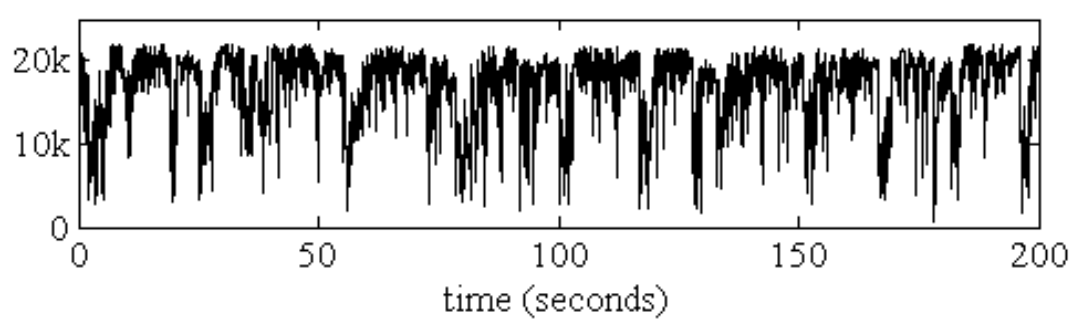

$\mathbf{F}_{\text {leak }}=$

0 L/min.

Figure 6. Effects of air bleed rate on sample throughput. 


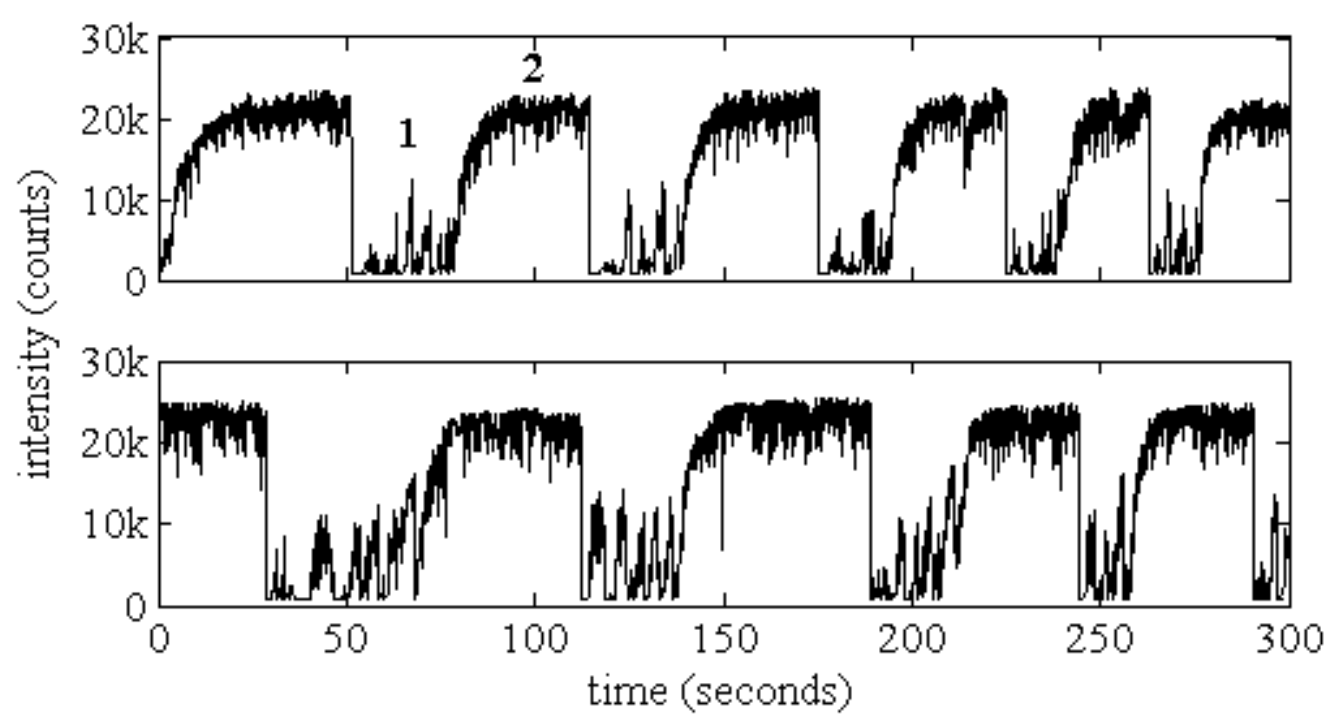

Figure 7. Sampler performance with $4 \mathrm{M}$ sodium nitrate in process tank.

air flow between sample slugs and changed the light throughput behavior. The periodic intensity dropouts in the top trace of Figure 7 (labeled (1)) coincide with solution exchange in the sample vial, and the intermittent periods with only air passing through the flow cell (2) show much less disturbance in the vial than does the less dense solution.* Note that even with the heavier solution, full air jet pressure and air bleed rates generate too much turbulence for adequate spectroscopy, and both factors must be diminished for optimal sampler operation with this solution. The excess capacity of the system suggests that successful operation with the denser solutions in Tanks 12.2 and 12.3 can be expected.

An examination of several field observations agrees with our interpretation of the effects of the operation parameter choices. The top trace of Figure 8 shows the performance of the sampler associated with Tank 16.8. The solution in this tank has a relatively high nitrate concentration $(\sim 4 \mathrm{M})$ but low salt content, and thus has a medium density for the range of solutions that will be analyzed with this spectrophotometer. The figure shows the intensity of light throughput at a wavelength $(600 \mathrm{~nm})$ where light is not expected to be absorbed versus time. The semi-periodic nature of the sample delivery is evident, where every 30-40 seconds a new slug appears, and the throughput decreases sharply until the entrained air can escape. This interpretation is corroborated by observations in the sample aisle, where a stream of bubbles appears in the sample vial at the same time that the intensity decreases. Between pulses, the throughput is relatively stable, and the solution in the sample vial is still, indicating that the air delivery is not intense. In the

\footnotetext{
${ }^{*}$ In the top trace of Figure 7, for 0-150 seconds, $\mathrm{P}_{\text {jet }}=60 \mathrm{psi}, \mathrm{F}_{\text {leak }}=0.4 \mathrm{~L} / \mathrm{min}$; for $150-300$ seconds, $\mathrm{P}_{\text {jet }}=$ $50 \mathrm{psi}, \mathrm{F}_{\text {leak }}=0.4 \mathrm{~L} / \mathrm{min}$. In the bottom trace, $\mathrm{P}_{\text {jet }}=48 \mathrm{psi}, \mathrm{F}_{\text {leak }}=0 \mathrm{~L} / \mathrm{min}$, simulated Hanford connector bleed rate $=0.3 \mathrm{~L} / \mathrm{min}$.
} 


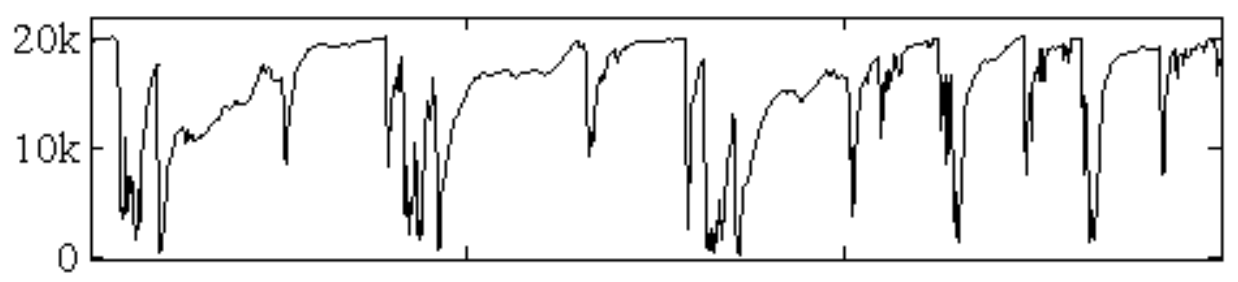

16.8

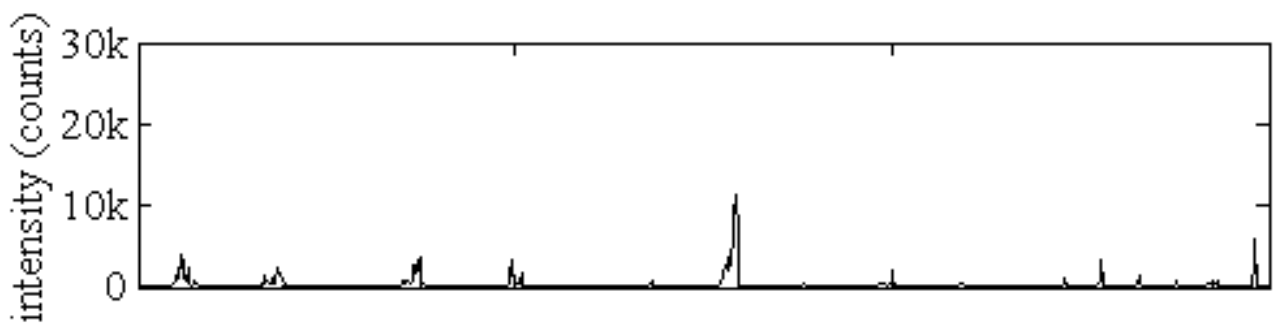

14.5(a)

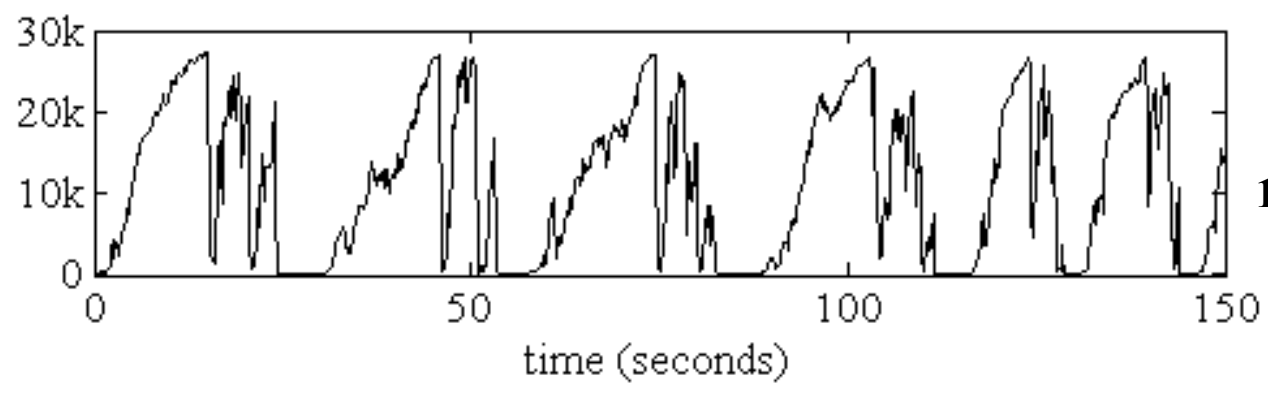

14.5(b)

\section{Figure 8. Sampler performance in H Canyon.}

absence of degassing or vortex equipment that could dissipate the entrained air before it reaches the flow cell, ${ }^{5}$ this sampler performance represents an ideal situation, in that there are time windows where acceptable spectra can be obtained.

Compare those results with the performance of the sampler associated with Tank 14.5, which is shown in the middle trace ("14.5(a)") of Figure 8. Tank 14.5 contains a lowacid, low-salt solution with a low density that does not require much air to lift to the flow cell. The trace shows the results for a situation where the air bleed valve was opened too wide, and too much air was introduced into the sample line. This trace represents constant turbulence, where the excess air is disturbing the solution in the flow cell (confirmed by observations of the sample vial). Turning down the jet pressure in this case did not change the performance significantly, but decreasing the air bleed valve to a "partially open" position did, as seen in the bottom trace ("14.5(b)") of Figure 8. There is, overall, more turbulence than for the Tank 16.8 sampler, but the regions of acceptable light throughput are long enough to obtain good data.

We simulated the effects of leaks at the Hanford junctions by closing the air bleed valve BV1 and opening either RV1 or RV2. We observed little difference in sampler performance with leak rates at these valves similar to those at the air bleed valve (for example, compare the top and bottom traces of Figure 7). The air inlet points for these valves are approximately 10-15 feet above the process vessel, and probably below the level where vacuum can draw the solution by itself. This behavior is also consistent with recent ob- 
servations of the sampler associated with Tank 18.7 in H-Canyon, for which leaks in the pipes are suspected. The turbulence in the sampler is too great for taking good data, even with the air bleed completely closed. This result indicates the importance of a continuous, leak-free line to proper sampler operation, especially if the air bleed rate must be restricted to minimize turbulence.

We examined the effect of heated solutions by passing the $4 \mathrm{M}$ nitrate solution through an external heat bath before returning it to the tank. The primary effect of heating was to reduce slightly the viscosity of the solution, which required a decrease of jet pressure and/or bleed rate to return sampler performance to the same rate as for the cooler solution. We also observed an increase in solution evaporation at the demister, although that did not affect sampler operation.

Proper sampler operation also includes effective sample exchange in the flow cell. Operational history in H-Canyon includes an instance in which using process water to flush a dense solution (approximately 6M nitric acid) from the flow cell required several hours. The problem occurred because the solution entry tube extended only a short distance into the " $T$ ", and the water did not enter the flow cell with enough force to displace the heavier solution at the bottom of the "T". The problem was solved by extending the tube to a point just above the top of the optical path defined by the collimating lenses. We verified that configuration by loading the process tank with water and the flow cell with $4 \mathrm{M}$ sodium nitrate and a small amount of Fluorescein green dye. The effectiveness of the sample exchange is shown in Figure 9. The typical spectral signature of the dye is removed within 2-3 minutes after exchange begins. The time frame for removal is consistent with a 2.5 minute removal time for a $7 \mathrm{~g} / \mathrm{L}$ uranium, $4-5 \mathrm{M}$ nitric acid solution with process water in H Canyon.

We examined the possibility that the optical path length chosen for the sampler would affect operation. Different path lengths may be chosen according to the solution absorption (large absorptions can be minimized with short path lengths), and the different geometry in the flow cell could change the dissipation of air (whether entrained with the liquid or passing through the cell between slugs) or the sample exchange rate. (Specific
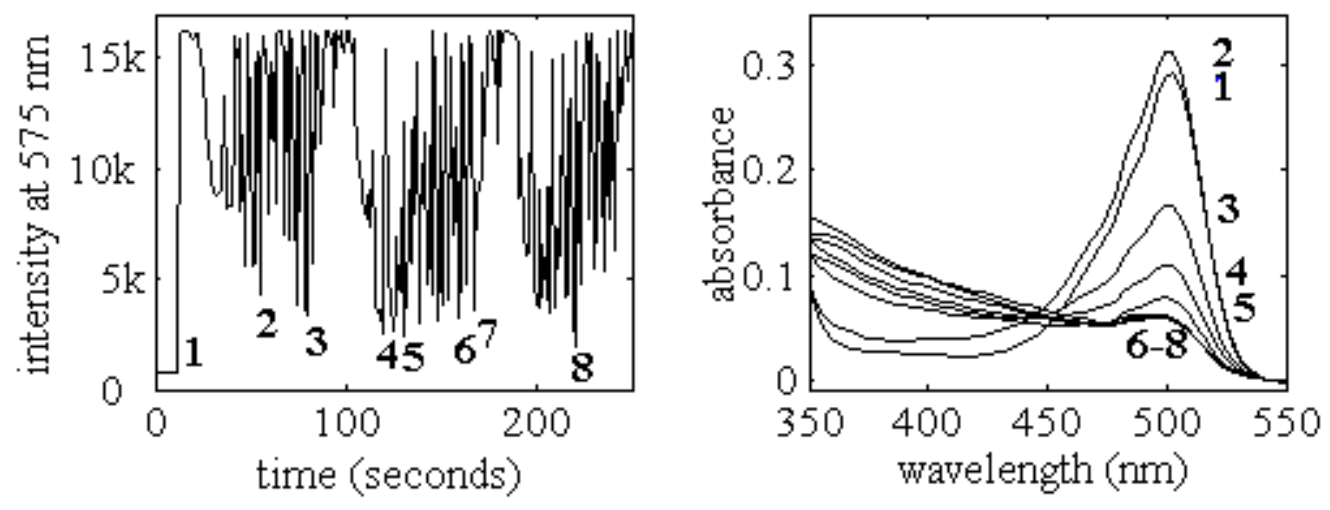

Figure 9. Sample exchange rate for flushing of $4 \mathrm{M}$ sodium nitrate with water. 
path lengths for this application will be discussed in the "Model" section.) In the normal configuration in which the " $\mathrm{T}$ " is not modified, there is little potential "dead space" between the lenses and the top arm of the "T". Therefore, for this or shorter path lengths, sample mixing should be rapid, and bubbles should not become trapped in the "dead space". Our observations from operating the sampler mock-up using a shorter path length confirm this reasoning. We did not see any effective differences in light throughput or sample exchange rate.

There are several broad conclusions that can be drawn from these studies. To the extent that the configuration of the sampler mock-up reflects the configuration in H-Canyon, the plant samplers should be able to effectively sample solutions at all the tanks to which the spectrophotometer will be applied. While there is a wide range of air jet pressures and air bleed rates that will allow sample collection, there is a narrower range of those parameters that will allow good spectrometer operation. Those settings are expected to be different for each sampler, due to differences in the solution density and temperature and sampler infrastructure leaks. After the sampler vial and flow cell assemblies are installed in the field, the jet pressure and air bleed rate for each sampler should be set by monitoring the time-dependent light throughput profile, as exemplified in Figures 5-8. Furthermore, those settings should be identified to assure reproducible sampler performance. Note that the settings may need to be adjusted as solution contents change with new process campaigns.

\subsection{Radiological effects of flow cell addition}

SRTC calculated the increased dose rate for personnel in the Hot Sample Aisle due to the volume of liquid contained in the flow cell part of the sampler assembly. Before the spectrophotometer project was started, only the sample vial was present in the box. The calculations are based on concentrations of fission products supplied by Nuclear Materials Management Division (NMMD). Details of the calculations may be found in SRTCADS-2001-0546 (F.S. Moore to C.W. Jenkins and R.J. Lascola, 12/13/01). Dose rates were calculated for two scenarios: (1) just outside the sampler with the sampler door closed, and (2) at a distance of 20 inches from the flow cell with the door open. Both the flow cell and vial are assumed to be 8.5 inches from the nearest sampler wall, which is 1.25 inches of steel. Dose rates due to the sample vial are 47 and $30 \mathrm{mR} / \mathrm{hr}$, and due to the flow cell are 51 and $32 \mathrm{mR} / \mathrm{hr}$, for scenarios (1) and (2), respectively. The flow cell and sample vial have nearly identical volumes, and thus the total dose outside the box is effectively doubled with addition of the flow cell. The on-line measurements will decrease the overall exposure of sample aisle operators and laboratory technicians, as fewer samplers will be collected and analyzed off-line. 


\subsection{Configuration effects of flow cell addition}

The samplers in the Hot Sample Aisle (e.g. Tanks 12.2 and 12.3) have a lead "doorstop" shield which normally covers the sample vial but swings open when the sampler box door is opened. It is impossible to retrofit the flow cell to this sampler without disconnecting the doorstop mechanism, which will shear the tubing. NMMD has determined that the fission product content for solutions in the HEU campaign is sufficiently low so that the doorstop can be disabled. Otherwise, the arrangement of the flow cell is similar to the setup at the Warm Sample Aisle. The flow cell is situated behind the sample vial, as viewed from the sampler door, to stay out of the way during vial removal and also be convenient for routing fiber optics. The sampler mock-up was configured to reflect this design.

\subsection{Flow cell path length}

For tanks located after the mixer-settler banks, low concentrations of potentially interfering species are expected. Therefore, the primary consideration for the optical path length of the flow cell is that it is long enough to obtain high signal-to-noise absorption spectra. For the expected concentrations shown in Table 1, the standard path length of 1 inch $(2.54 \mathrm{~cm})$ is acceptable, and no modification of the compression fitting is required. However, for Tanks 12.2 and 12.3, a long path length can be problematic if the interfering species absorb too much light and obscure the uranyl nitrate absorptions. For these tanks a path length of 0.6 inches is appropriate. A smaller pathlength is precluded by the length of the collimating lenses, as the ferrules in the compression fitting must mate with the lens body. As will be shown in the section describing the prediction models, the expected interferents will not obscure the light, and the uranyl nitrate absorptions are adequate. The flow cells which will be used for Tanks 12.2 and 12.3 have been drilled out so that the collimating lenses will seat at the appropriate separation. The flow cells may be distinguished by the stamp " 0.6 ".

\section{DATA ACQUISITION PROGRAMS}

\subsection{Introduction}

Almost all aspects of spectrophotometer operation are contained within the program Onlinez.exe. The program performs the following major functions:

- Set and run a schedule of multiple sample point analyses in a continuous mode.

- Set and control the optical multiplexer and diode array spectrometer, and read data from the spectrometer.

- Support several chemometric models for multiple component determination, e.g. uranium and acid, from a single spectrum.

- Analyze spectral data and calculate concentrations based on models and determine statistical error and 'goodness of fit'. 
- Monitor light intensity and communications with the multiplexer and spectrometer to ascertain proper instrument function.

- Plot spectral and concentration results data.

- Store data for off-line analysis, and time- and date-stamp model results.

- Send (analog) concentration data and (digital) alarm information to the Field Point I/O system.

There is a second program, Watchdog.exe, which sends an alarm signal to a reserved channel on the Field Point system if Onlinez.exe is no longer active. Both programs are written in the Visual Basic platform (versions 5.0 and 6.0) and have been tested with Windows 95, 98, NT, and 2000 operating systems. (Currently, the NT and 98 platforms are used by the computers associated with spectrophotometers 1 and 2, respectively, in $\mathrm{H}$ Canyon.)

The following sections describe the information required to use the programs, interpret the alarm signals that the programs generate, and modify program parameter data files which control program operation.. The information is presented in the order that the functions are most likely to be encountered. However, a knowledgeable user of the instrument should be aware of all of the program functions. Those who require more information concerning program operation are referred to Appendix 1, which contains a detailed description of the modules and forms used in the program.

\subsection{Program Operation}

Both programs are started by running shortcuts from the Windows desktop. Running the Onlinez.exe shortcut generates the start screen, which is shown in Figure 10(a). This form of the startup screen presents only two obvious options to the user and prevents an inadvertent change to the configuration files. Clicking the green "Go" button starts the program. The multiplexer will switch channels, first reading channel 0 , which is a null channel that allows the computer to read spectrometer noise levels that will be subtracted from the data. It will next read channel 1, which is the reference channel, and then will read the channel specified in the sampling schedule. Eventually several new windows will appear on the computer monitor. ${ }^{\dagger}$

Windows which will appear every time the program is started include a table of results for the different channels/tanks that are monitored and a display of the most recently acquired spectrum. A typical monitor display is shown in Figure 11. Details of the results table include the channel and tank identities, uranium and acid concentrations, and the time of the last analysis. Details of the spectrum screen include the plot of absorbance versus wavelength, the channel number associated with the displayed spectrum (located in the left margin), and a red "Stop" button used to halt the data acquisition (located in

\footnotetext{
* We have found that versions of Onlinez compiled on one computer may not run on a second computer, and thus recommend that the source code be installed on and compiled with each new computer that will be running the program.

${ }^{\dagger}$ The windows may be dragged to different parts of the screen so they do not overlap.
} 


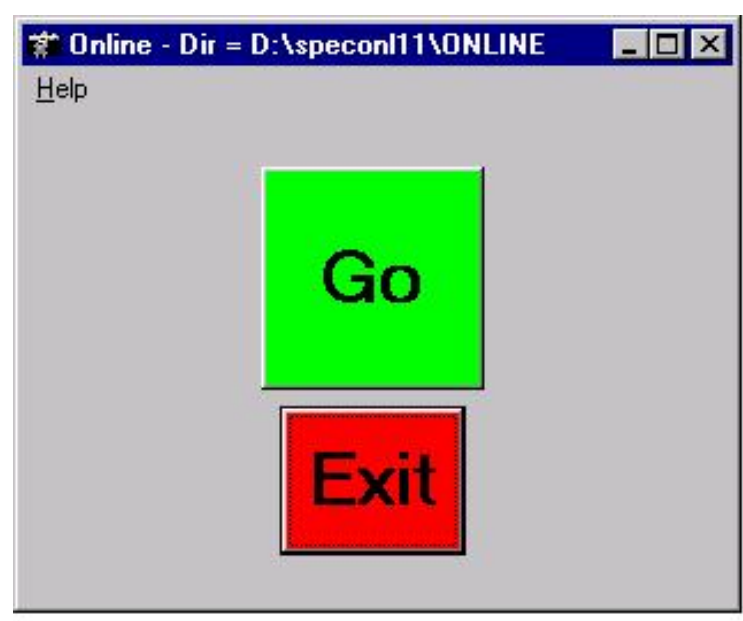

(a) Start screen without extra options.

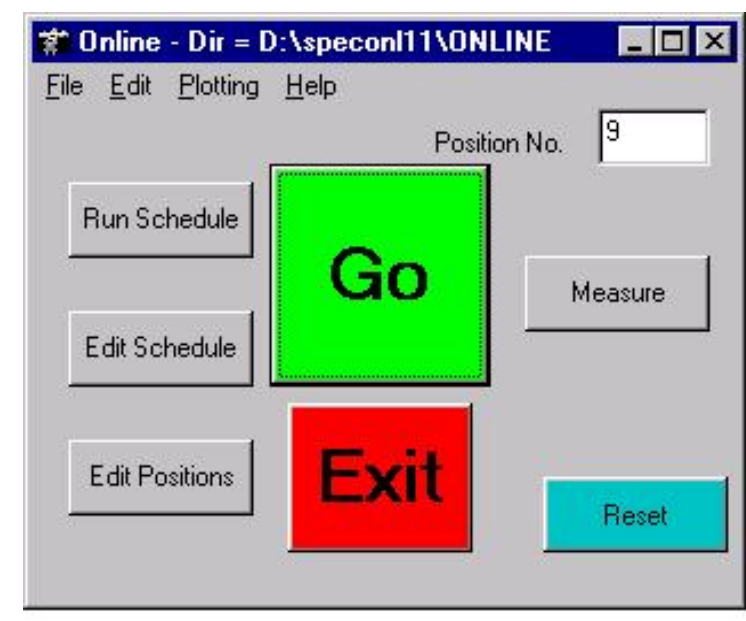

(b) Start screen including extra options.

\section{Figure 10. Startup screens for data acquisition program Onlinez.exe.}

the upper right corner). Figure 11 also shows the window associated with Watchdog.exe. Displayed in that window are the time, digital value sent to the Field Point unit, and an "Exit" button.

Another window which may appear is a message log file, which records specific conditions associated with sending an alarm to the Field Point modules. The log file only appears when an error is detected. Once the window appears, it cannot be closed until the program has been stopped, though it can be minimized. Also available, but currently disabled, are time trace windows for the results associated with each channel. These can be activated within the schedule.dat data file described below. However, with multiple tank analyses, these windows clutter the computer display. As the information presented in these windows can also be obtained from the H-Canyon DCS, the time trace windows are not generally used.

Stopping the data acquisition program is straightforward but must be done in a certain order. First, exit Watchdog.exe. Next, click the "Stop" button on the spectral display screen. Besides stopping data acquisition, this action also disables a separate watchdog timer on the Field Point control module which normally monitors two-way communication with the data acquisition program. If the program is actively reading a channel, the spectral screen will appear again. If this happens, click the "Stop" button a second time. Then click the "Exit" button on the original startup screen to exit the program.

\subsection{Alarm signals}

The data acquisition program is sensitive to the malfunction of all the individual components of the spectrophotometer (lamp, computer, spectrometer, and multiplexer), and will respond to conditions associated with fiber optic breaks, occluded flow cells, and poor 


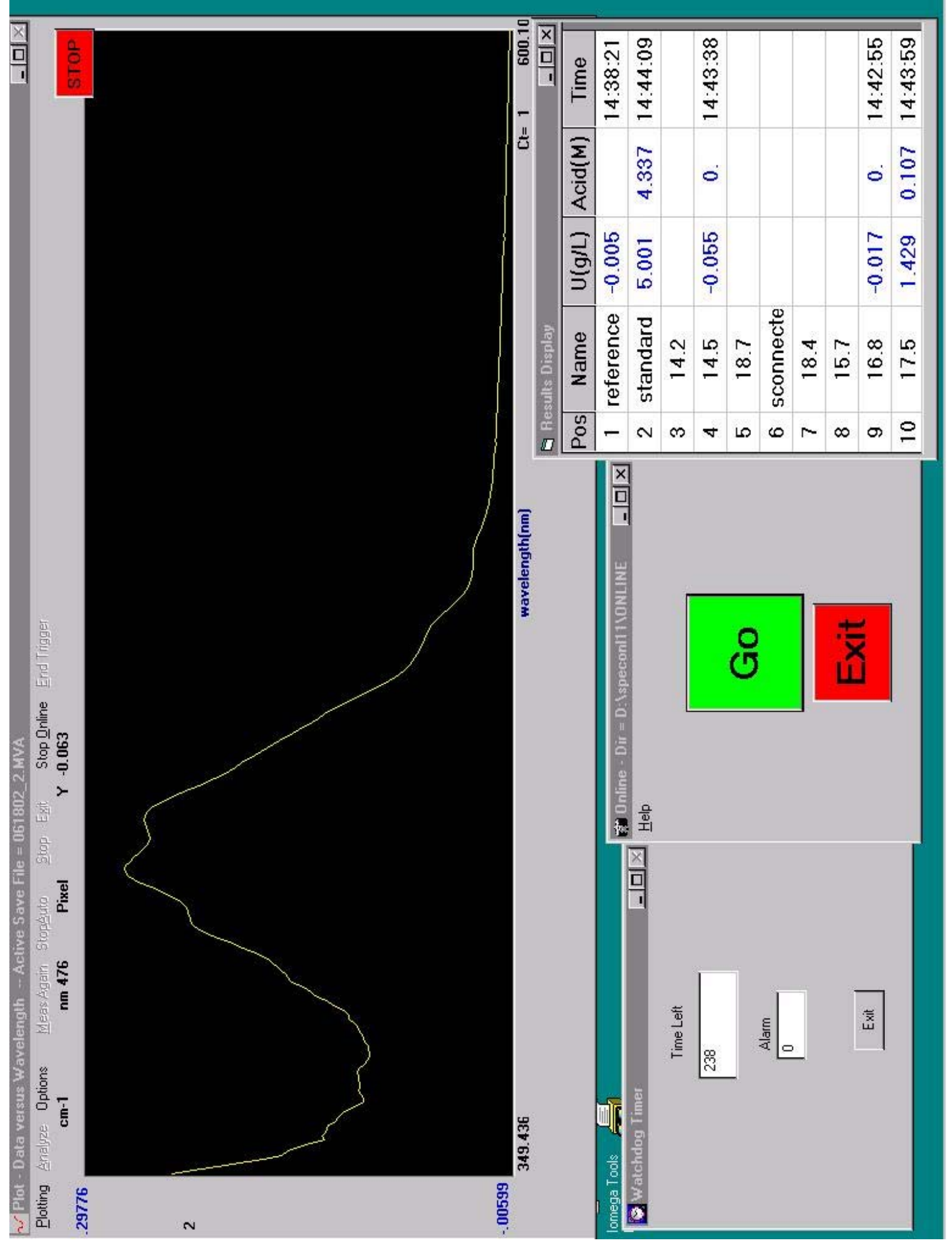

Figure 11. Display during operation of Onlinez.exe. 
sampler operation." Each alarm signal only indicates that there is a problem associated with data acquisition for a specific channel (or with the system as a whole, if the watchdog alarm is activated). Thus, the identity of the specific component that has failed must be deduced from the pattern of alarms that activate and the messages that appear in the message box. ${ }^{\dagger}$ The potential failure modes and the expected instrument response are listed below.

Lamp failure. Low light levels are monitored by noting the intensity of light present at a wavelength where light absorption by process solutions are low. For this application, neither uranyl nitrate nor the expected interferents (iron, mercury, etc.) will absorb in the region between 550 and $600 \mathrm{~nm}$; the intensity at a wavelength in this region is evaluated. The region is defined in the instru.dat data file. After a failure, the alarm will activate for each channel that is subsequently read, and for both outputs (uranium and acid) associated with that channel. The message log will note that low light levels have been observed at that channel, that "no good data" has been collected, and that the "residual" and "uncertainty" threshold values for the analysis have been exceeded. (These responses are mostly relevant to sampler operation and will be discussed in more detail below.)

Cabinet fiber optics failure. Two fiber optics are completely contained in the equipment cabinet. These connect the multiplexer to the lamp and the spectrometer. Since these fibers are used during each measurement, their failure will produce the same response as the failure of the lamp.

Multiplexer failure. While unlikely, the multiplexer unit may fail to switch properly or respond to the computer control. This failure is addressed in two ways. The data acquisition program requests the multiplexer to send a response after receiving and executing a command. If the response is not sent, the program produces alarm signals for the channel being read and writes a "multiplexer not responding" note in the log file. Also, multiplexer failure is likely to produce unusably noisy data, because the detector noise signal will be the same magnitude as the reference and sample data. Thus, "high residual" and "high uncertainty" notes may also appear in the message log. In some cases, a divide-byzero error may occur, if the detector noise signal at one wavelength happens to be exactly the same as the reference signal. A divide-by-zero error will cause the program to crash, and eventually activate the watchdog alarm (see "program failure").

Spectrometer failure. Spectrometer failure is unlikely. However, occasionally short communication dropouts are observed. The source of these dropouts is unknown, though they are thought to be correlated to power line noise. The dropouts are caught by drivers for the National Instruments input/output card. Normally, the driver response would be to display a dialog box requiring user input to continue. While the dialog box is displayed, the watchdog timer continues to count down, and the watchdog alarm will eventually sound. To prevent these nuisance alarms, the response has been altered so that the dialog box is displayed when the fourth dropout observed before fifty normal spectra are

\footnotetext{
${ }^{*}$ Failures of the Field Point modules (open circuit detection, module failure) are monitored by the DCS and are outside the scope of this document.

${ }^{\dagger}$ The messages are not transmitted to the DCS.
} 
taken. In this way, transient dropouts can be overcome without reducing the effectiveness of the built-in error checking. The ratio of normal spectra to dropouts is defined in the spec.alm data file. Since spectrometer failure halts the data acquisition program, there should not be any alarms associated with a specific channel.

Computer failure. Computer problems may arise from the data acquisition program or the computer hardware. As implied in the spectrometer failure discussion above, a program failure will be detected by the Watchdog.exe program. The watchdog program will alarm if it does not receive a signal from the main program within four minutes. This interval is defined in Watchdog.exe and cannot be changed without recompiling the program. A total computer failure (loss of power, reboot, etc.) will stop both the Onlinez.exe and Watchdog.exe programs. This failure is detected by a watchdog feature in the Field Point control module. This interval, currently set to five minutes, is defined in Onlinez.exe and communicated to the control module when the "Go" button is clicked. The watchdog is disabled when the "Stop" button on the spectral display window is clicked.

Fiber optic breaks in sample aisle. Errors associated with loss of fiber optic continuity to a sampler (or reference or standard cell) will give alarms only on the affected channel. The messages written to the log file will be "low light" and possibly "no good data" and "high residual" and "high uncertainty" for that channel. Divide-by-zero errors should not occur unless the fibers connected to the reference cell are damaged (in which case the dark current and reference spectra would be comparable).

Flow cell occlusion. A complete loss of light through the flow cell will give the same pattern of alarms and errors as the fiber optic breaks described above. The lenses could discolor due to the deposition of a colored film, which could affect the quality of the data. ${ }^{*}$ Coloring would only produce an instrument response if it resulted in excessive fit uncertainties or fit residuals. These conditions would give data alarms and the appropriate message log entries. The presence of a colored film could be deduced from observing a change in the background of the spectrum, or more definitively, from observing the absorption spectrum of process water poured into the sampler.

Sampler malfunction. As discussed at length in the Section 3, improper sampler settings can lead to excessive turbulence in the flow cell which prevents the acquisition of meaningful data. Even with proper sampler operation, there are still times when the turbulence is too large. The turbulence does not appear periodically, so the program must evaluate the quality of the data immediately after it is acquired. Turbulence leads to a spectral baseline offset that is independent of wavelength, and thus can be monitored in the same way as lamp failure is monitored, by observing the intensity of light in a region of the spectrum where the solution is not expected to absorb. Individual spectra are evaluated, and those with enough light intensity are retained for processing. The program keeps count of the number of accepted and rejected spectra for each analysis, and compares the counts to target values. The thresholds for spectral retention intensity, as a fraction of intensity through the reference channel, and number of acceptable (or "good")

\footnotetext{
* The current procedure to rinse the sampler with process water after each operation has greatly diminshed the appearance of films on the Tank 14.5, 16.8, and 17.5 samplers.
} 
spectra to obtain, i.e. the number of spectra to average, are defined in the options.dat file for each channel. The target number of unacceptable (or "bad") spectra is defined in spec.alm. Complete descriptions of these files are found in Section 4.4.

There are three scenarios which can occur. The ideal (and usual) situation is that the target number of good spectra are obtained before the target number of bad spectra. Analysis proceeds and results are reported normally to the DCS. A less ideal situation is that some good spectra are obtained, but fewer than desired before the bad data count is reached. In this case, data are analyzed and reported normally, with no alarms, but a note is recorded in the message log. This approach reflects the fact that the analysis result is still valid, but may be less precise than the result from a larger data set. The worst situation is that no good data is collected before the bad data threshold is reached. In this situation, the computer holds the results of the previous analysis, activates the data alarm to the DCS, and writes the "no good data" and poor analysis messages to the log file. The previous good analysis result is held to prevent the display of a meaningless value on the DCS that could be interpreted as a process upset and provoke an unnecessary response from control room operators.

\subsection{Data files and settings}

There are several data files which preserve the proper instrument configuration. Some of these files can be edited within the context of Onlinez.exe from the start screen. The keystroke sequence $<$ CRTL $>$ - M activates a hidden menu bar which allows access to channel options, schedule, and instrument (spectrometer) files. The appearance of the start screen changes, as shown in Figure 10(b). The sequence $<$ CTRL $>$ - O hides the menu bar. All the data files described below can also be accessed using a standard text editor. Some important Field Point settings are contained in the configuration file Mark1.iak. Doubleclicking on this file starts the National Instruments configuration utility.

Schedule.dat This file contains data which determines the order in which the various positions are monitored and the interval between measurements. The typical view from the context of Onlinez.exe is shown in Figure 12. The program uses the channel numbers to pick the analysis options file (see below) and multiplexer setting; the labels corresponding to tank numbers are for information only. After each measurement, the program reads schedule.dat line by line. If the specified measurement interval (displayed in seconds) for the first active line has elapsed, the measurement is made for that channel, and the time stamp for that measurement is stored. If the interval has not elapsed, the program reads the next line and makes the same evaluation, continuing until all active lines have been evaluated. If the program goes through all lines without making a measurement, it returns to the top of the file and repeats the process.

The intervals and priorities must be selected carefully, as a channel with a high priority and short interval could prevent other channels from ever being read. The reference and standard channels are monitored solely to assure instrument function, and can be measured less frequently and with lower priority than other channels. Channels with noisy 


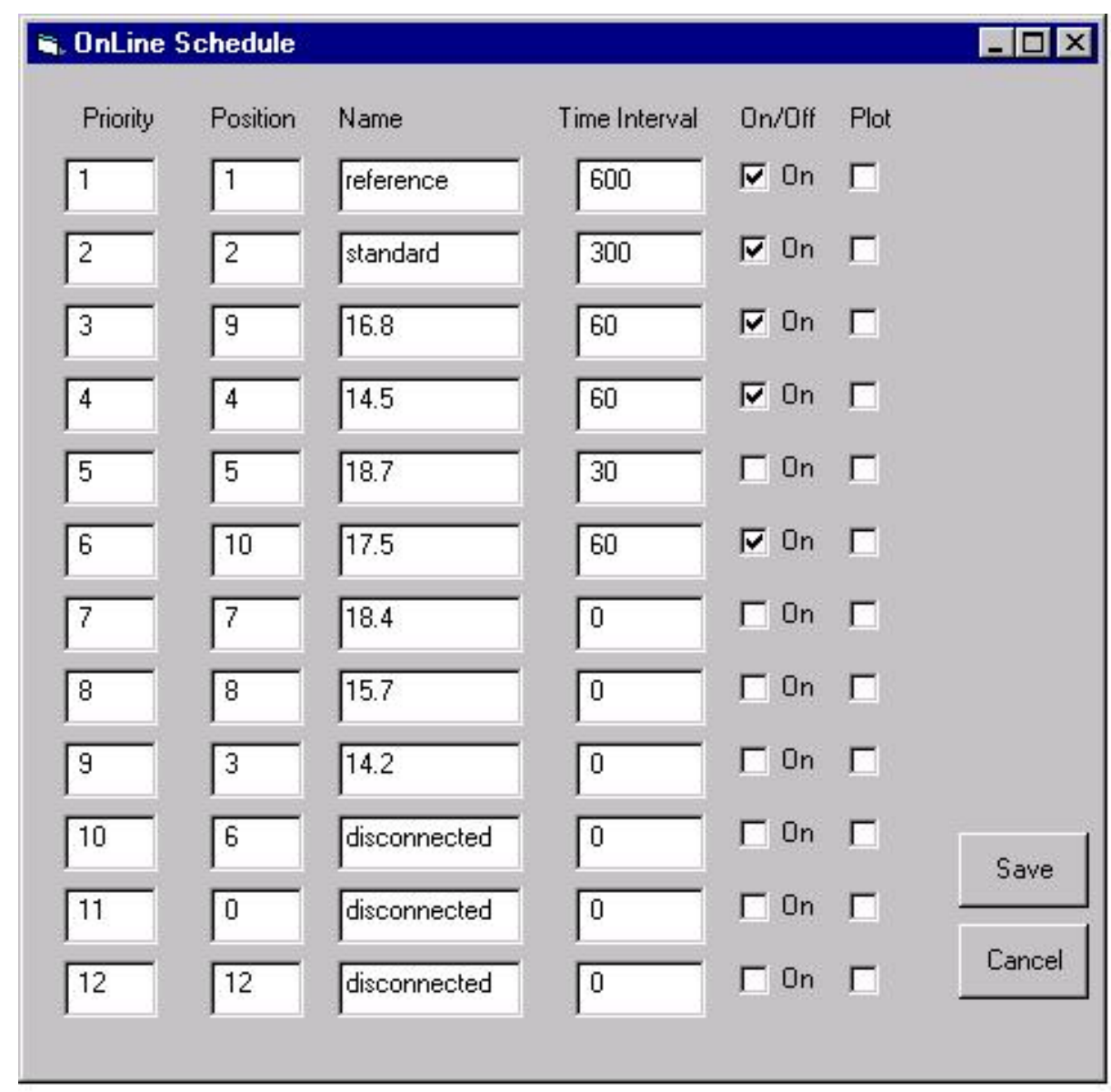

\section{Figure 12. Schedule display window (example).}

sampler operation will require a longer period of time to monitor, as a larger number of bad spectra must be read and rejected before the good spectra quotient will be reached. When setting up the schedule, operation should be observed for an extended period of time with the samplers running to assure that all channels are being read. Two- minute intervals are adequate for monitoring three channels with normal sampler behavior in addition to the standard and reference channels; longer periods may be required if more channels are added to the spectrometer. The "Plot" checkboxes control the time trace windows described in Section 4.2.

Options\#\#.dat. The wildcard '\#\#” corresponds to the channel number (e.g. Options4.dat, Options 10.dat). This file contains information that is specific to the tank being sampled. All options are shown on the display screen in Figure 13. Important options and their significance are discussed below. (Other options are inactive.)

- MPlex Pos. Multiplexer channel number, as defined by the program. 


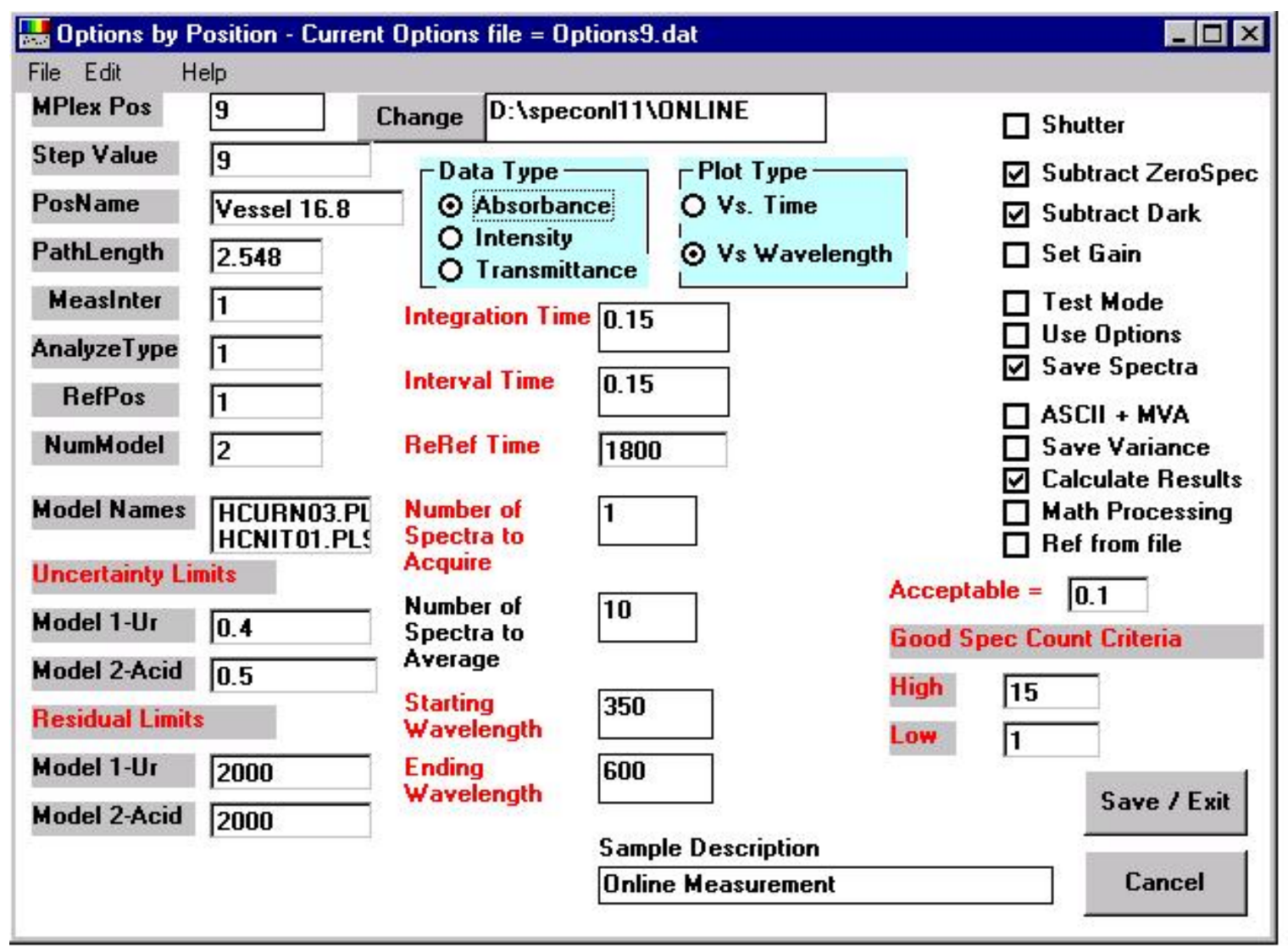

\section{Figure 13. Options display window (example).}

- $\quad$ Step Value. Multiplexer channel number, as defined by the multiplexer..

- PosName. Label for channel number. (Arbitrary value for display purposes only.)

- PathLength. Factor to account for path length differences relative to path length used for calibration model. (For the HEU spectrophotometer application, all calibration data were obtained with a $1 \mathrm{~cm}$ cuvette. Most of the sampler flow cells have an approximately 1 inch path length, so for those points, the corrective factor is 2.54 (results are divided by this value).)

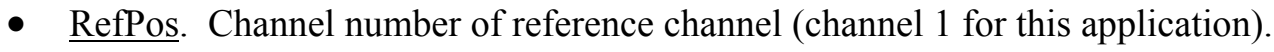

- NumModel. Number of chemometric models to apply to the data. Currently, the program can accommodate up to two models.

- Model Names. File names (e.g. HCNIT01.PLS) of first and (if necessary) second models. Model names must be separated by a comma, with no spaces. Names are not case sensitive. For this application, the uranium model is always the first model, and nitrate is always the second model. Models must be created using the Mvasrs.exe analysis program, which presents the calibration information in the appropriate format.

- Uncertainty Limits, Residual Limits. Threshold values for fit uncertainties and residuals which are generated in the course of data analysis. Values are in appropriate units for each model, e.g. g/L for uranium and M for nitrate. Recall that "Acid" is synonymous with nitrate for this application. Uncertainties or residuals that exceed these values indicate that the analysis may be suspect. Often, the conditions that lead to this scenario arise from unexpected changes in the solution components. Usually, additional diagnostic work is required to determine the true source of the problem. These limits are based on spectrometer perform- 
ance in the laboratory and in the facility. For channels where chemical interferents are expected to be high, the residual limits could be much greater than for relatively clean channels.

- Data Type. Set to "Absorbance" for normal operation. Can be set to "Intensity" if diagnostic information is being gathered (see "Integration Time"). The "Calculate Results" and "Math Processing" boxes should be unselected if "Intensity" is selected.

- Plot Type. Set to "Vs. Wavelength".

- Integration Time. Defines how long (in seconds) the array will be exposed to light and accumulate signal. Times are set during installation to balance the following factors. Higher intensities provide better precision, but can lead to detector saturation if made too high. Light sources with strongly structured output such as a Xe arc lamp can saturate small wavelength regions, which can lead to spectral artifacts. At least 1000 counts above dark current levels should be collected in the important wavelength regions; ideally, 20-30,000 counts will be obtained with a nonturbulent, nonabsorbing solution, which allows some loss of light from scattering and absorption without reducing data quality. Shorter times reduce the possibility of obtaining data with high turbulence levels in the flow cell (i.e. the turbulence in the cell does not change in the middle of reading the spectrum, and the data is definitely good or bad). Typical values for the integration time for this application are $0.1-0.25$ second.

- Interval Time. Defines how long (in seconds) the program should wait between successive reads of the spectrometer. This option is meaningful for the stand-alone version of Onlinez.exe which does not have an external schedule routine. For this application there is no need to wait, so typical values are 0.1 second.

- $\quad$ ReRef Time. Defines how long an interval (in seconds) should pass before a new reference spectrum is acquired. In H-Canyon, the instrument is intended to obtain a new reference with each measurement, and thus this value is set to 1 second.

- $\quad$ Number of Spectra to Acquire. Set to 1.

- Number of Spectra to Average. Self-explanatory. Setting this value must balance between improving noise levels and the time required to obtain the measurement. Typically, $25 \mathrm{spec}-$ tra are averaged.

- $\quad$ Starting Wavelength; Ending Wavelength. The spectrometer can read from approximately $185-1100 \mathrm{~nm}$. It is unlikely that all the information contained in that spectrum is required for analysis. These values allow the user to restrict the information pulled from the diode array, reducing the size of the data files which are saved. The limits also define the x-axis for the spectral display window. These values must include the wavelength range(s) of the model(s), as well as the range of data used to determine baseline offset for alarm activation. Also note that if the data is derivatized in the model, there will be a series of leading or trailing zeroes that must be outside the model wavelength range. For this application, a range of 350-600 $\mathrm{nm}$ is sufficient.

- Acceptable. This is the fraction of intensity of the reference channel that is acceptable for the channel being monitored without rejecting the data as too turbulent. Typical values are 0.10.2 .

- Good Spec Count Criteria (High and Low). These values are currently disabled. Their function, to be the threshold limits for the data selection routine described in the "sampler malfunction" section above, are filled respectively by the "Number of Spectra to Average" value in this file and the "MaxBadDataCount" value in spec.alm.

- Subtract ZeroSpec (checkbox). Allows use of baseline zero file (see description of zero\#.mva below). Must be checked for all channels except channel 1.

- $\quad$ Subtract Dark (checkbox). Allows subtraction of dark current (channel 0) from results. Must be checked for all channels. Failure to do so will skew high absorbance readings. 
- Save Spectra (checkbox). Allows spectra to be saved in mva format, which can be read by the Mvasrs.exe analysis program. See discussion of data output files below for more information.

- $\quad$ ASCII + MVA (checkbox). This feature will save spectra in ASCII as well as mva formats. The ASCII format is required to read the data into an Excel spreadsheet. This option is normally not checked. The conversion of ASCII to $m v a$ can also be done manually within the stand-alone version of the data acquisition program. Also, the data can be read into the Matlab environment using a special $m$ function. Saving the data in the additional format merely fills up the computer hard drive faster.

- Calculate Results (checkbox). This box must be checked to force the program to apply the models specified in the "Model Names" box.

- Math Processing, Ref from file (checkboxes). These boxes must remain unchecked. Their functions are replicated in other parts of Onlinez.exe. Math processing strings are written into the model files. New references are obtained with each measurement.

Instru.dat. This file defines operation parameters that are common to all measurement points, including information about the spectrometer wavelength calibration, multiplexer COM port, and lamp check intensity level. As with the options.dat files, there are several legacy entries from prior versions of the program that are not used in Onlinez.exe. The relevant entries are described below.

- Max Pixels, Quad Factor, Lo Wavelength Limit, Increment. These values define the wavelength calibration of the spectrometer. Both spectrometers contain 1024 pixels. The other values are determined by obtaining the spectrum of the output of a mercury/argon lamp, and fitting the pixel positions of the intensity peaks to reference wavelength values. The Lo Wavelength Limit defines the wavelength (in $\mathrm{nm}$ ) of the first pixel, the Increment describes the linear pixel increase, and the Quad Factor contains any quadratic corrections. The wavelength definitions for each pixel must be identical to those defined in the model files, or an error will be displayed. Note that the Hi Wavelength Limit entry is not used by the program.

- Multi Com Port. This value defines the COM port through which the program communicates with the multiplexer. For both computers COM port 2 is used.

- Reference Position. This value is superceded by the similar setting in the options.dat files.

- $\quad$ Number of Multiplexer Positions. Equals '12' for both instruments.

- $\quad$ Check Wavelength, Check Intensity Level, Check Position. The check for lamp function occurs in each measurement cycle during the reference spectrum acquisition; hence the check position is always set to ' 1 '. The check wavelength is set in a region where the process solutions will not absorb (currently set to $580 \mathrm{~nm}$ ). The intensity level is set to a value below which data obtained at other channels may not be reliable even with normal attenuation due to fiber differences and sampler operation (currently set to 2000 counts).

Spec.alm. This small file contains parameters for routines described in the sampler malfunction section above. MaxBadDataCount is the threshold number of spectra obtained with excess turbulence in the flow cell, for which the results are not reliable because too much light has been scattered. It is currently set to 300 spectra. WaveStartAve and WaveEndAve define the wavelength region over which the baseline intensity is calculated. These are currently set at 550 and $600 \mathrm{~nm}$, respectively.

Zero\#.mva. The "zero file" is a spectrum which is a measure of the differences in fiber, flow cell, and multiplexer transmission for each position compared to the reference cell. 
It is different for each channel, and allows the apparent absorptions due to those differences to be subtracted out. It is obtained for each position by recording the spectrum of water that has been poured into the flow cell (or for the standard cell, for a water-filled cuvette that has been placed in the cuvette holder). The zero\#.mva file (where \# is a placeholder for the channel number) must contain all 1024 pixels, since the program reads and calculates the absorption spectrum for the entire diode array before truncating the data and performing other math functions.

Model files. The model files (generally of the form *.PLS, *.PCR, or *.WAV) must be generated using the Mvasrs.exe data analysis program. For this application, there is a naming convention which must be followed. The nitrate analysis incorporates the results of the uranium analysis in its calculations. The incorporation occurs after the analysis in the $P L S$ file has been calculated. The trigger for the incorporation is embedded in the name of the nitrate PLS file, which must be of the form ??NIT??.PLS. ${ }^{*}$ Details about the models are provided in Section 5. As will be appreciated from that discussion, the calculations are complicated, and to assure good spectrometer performance the model files should not be altered. In particular, the models are spectrometer-specific - a model developed for one instrument will not give reliable results for another instrument - and the model files must not be moved between computers.

National Instruments files. The Field Point modules must be configured so that the output range of the devices matches the expectations for the DCS. The information is stored in the configuration file Markl.iak. The most critical parameters involve the ana$\log$ outputs. The total signal range is $3.5-21 \mathrm{~mA}$, with a power-up value of $3.5 \mathrm{~mA}$. Note that the total range is not used to report uranium and acid concentrations, as the DCS reserves the lower section of the range for diagnostic purposes. The data acquisition program translates results to $6-20 \mathrm{~mA}$, with uranium concentrations ranging from $0-15 \mathrm{~g} / \mathrm{L}$ and acid values from 0-10 M. The 3.5-6 mA range is used by the DCS for diagnostic purposes. These settings are hard-coded into Onlinez.exe, and are not user-adjustable.

Output files. Output files include spectra files, analysis log files, and message log files. Spectra file names are of the format mmddyy_\#\#.mva (and *.std), where mmddyy is the standard month-day-year convention and \#\# is the position number. Typical file sizes for a channel read every two minutes, with data from 350-600 nm, are 2.0-2.3 MB for the $m v a$ file and $40-45 \mathrm{kB}$ for the std file. Analysis log files for uranium and nitrate are named $U r \_$pos\#\#_mmddyy.log and Nit_pos\#\#_mmddyy.log, respectively. For the same schedule just described, each file will be $45-50 \mathrm{kB}$. Each line in the analysis files contains the position number, analyte, value, fit uncertainty, fit residual, data, and time. Message files are named Mmddyy.log.

The "first" spectrometer has a smaller hard drive than the second. We have found that the spectra and results files must be removed from the instrument approximately every 35 months, depending on the number of channels measured. SRTC will provide a batch file that will allow this operation to be performed by NMMD personnel.

\footnotetext{
${ }^{*}$ The ? is a single-character wildcard; example filenames which meet this format are HCNIT21.PLS and HCNIT04.PLS.
} 


\section{PREDICTION MODELS}

\subsection{Introduction}

The chemistry and UV-visible absorption spectroscopy of uranium(VI) in nitric acid is well-established. ${ }^{1,6,7}$ The uranyl ion, $\mathrm{UO}_{2}{ }^{2+}$, forms a series of complexes with free nitrate ions. The equilibrium between $\mathrm{UO}_{2}{ }^{2+}, \mathrm{UO}_{2} \mathrm{NO}_{3}{ }^{+}$, and $\mathrm{UO}_{2}\left(\mathrm{NO}_{3}\right)_{2}$ is determined by the relative concentrations of the uranium and nitrate. ${ }^{8,9}$ For the concentration range defined by the scope of this work, the relative amounts of these species do not depend on uranium concentration, but do depend strongly on the nitrate concentration. This dependence is reflected in the absorption spectra of the system, which are shown in Figure 14. The absorbance peaks scale linearly with uranium concentration through $11 \mathrm{~g} / \mathrm{L}$. (Other researchers have established that the linearity extends to at least $30 \mathrm{~g} \mathrm{U} / \mathrm{L}$ for this range of nitrate concentrations. ${ }^{10}$ ) However, the absorbance line shapes and intensities change dramatically with changes in nitrate concentration, especially above $1 \mathrm{M}$.

The spectrum of the solution is affected by temperature. Figure 15 shows the spectral changes associated with a change of temperature from $20-45^{\circ} \mathrm{C}$, which represents the range of temperatures which may be found in the various tanks in the process. While the spectral changes due to temperature are small compared to those associated with nitrate concentration, they are still significant (extinction coefficients increase by $6 \%$ in the wavelength regions on which prediction models are based, shown by the arrows) and can affect the analysis for uranium and nitrate concentrations. Furthermore, the temperature of the solutions in the flow cell is unmeasured and uncontrolled, as the solution can exchange heat with the pipes as it is carried to the sampler. Therefore, the analysis will have to be insensitive to temperature.

The spectral effects of various metal ions are even stronger than the temperature effects. Metal ions may be introduced to the solution either intentionally, as part of the control of the chemistry of the process, or inadvertently, from degradation of the pipes or as byproducts of the fuel dissolving process. Spectra of several typical species are shown in Figure 16. The effects of colored species such as $\mathrm{Fe}^{2+}, \mathrm{Fe}^{3+}, \mathrm{Hg}^{2+}, \mathrm{Cr}^{3+}$, and others are obvious, as they absorb in the same region as the uranyl species (shown by the arrows). Less obvious are the potential effects of colorless species such as $\mathrm{Al}^{3+}$, which is present in potentially high concentrations (up to $3 \mathrm{M}$ ) that can affect the ionic strength of the solution. Subtle spectral effects may subsequently arise from changes in the spheres of hydration surrounding the analyte. ${ }^{11}$ Note that uranium oxidation state is not expected to change in these solutions due to the presence of $\mathrm{Fe}^{2+}$.

Currently, colorimeters using two wavelengths to measure concentration are installed at Tanks 14.2 and 15.7. These instruments measure light intensity at wavelengths where uranium absorbs light $(415 \mathrm{~nm})$ and is transparent $(540 \mathrm{~nm})$. Uranium concentration is calculated from those measurements by a formula derived from Beer's Law (Section 2.1): 

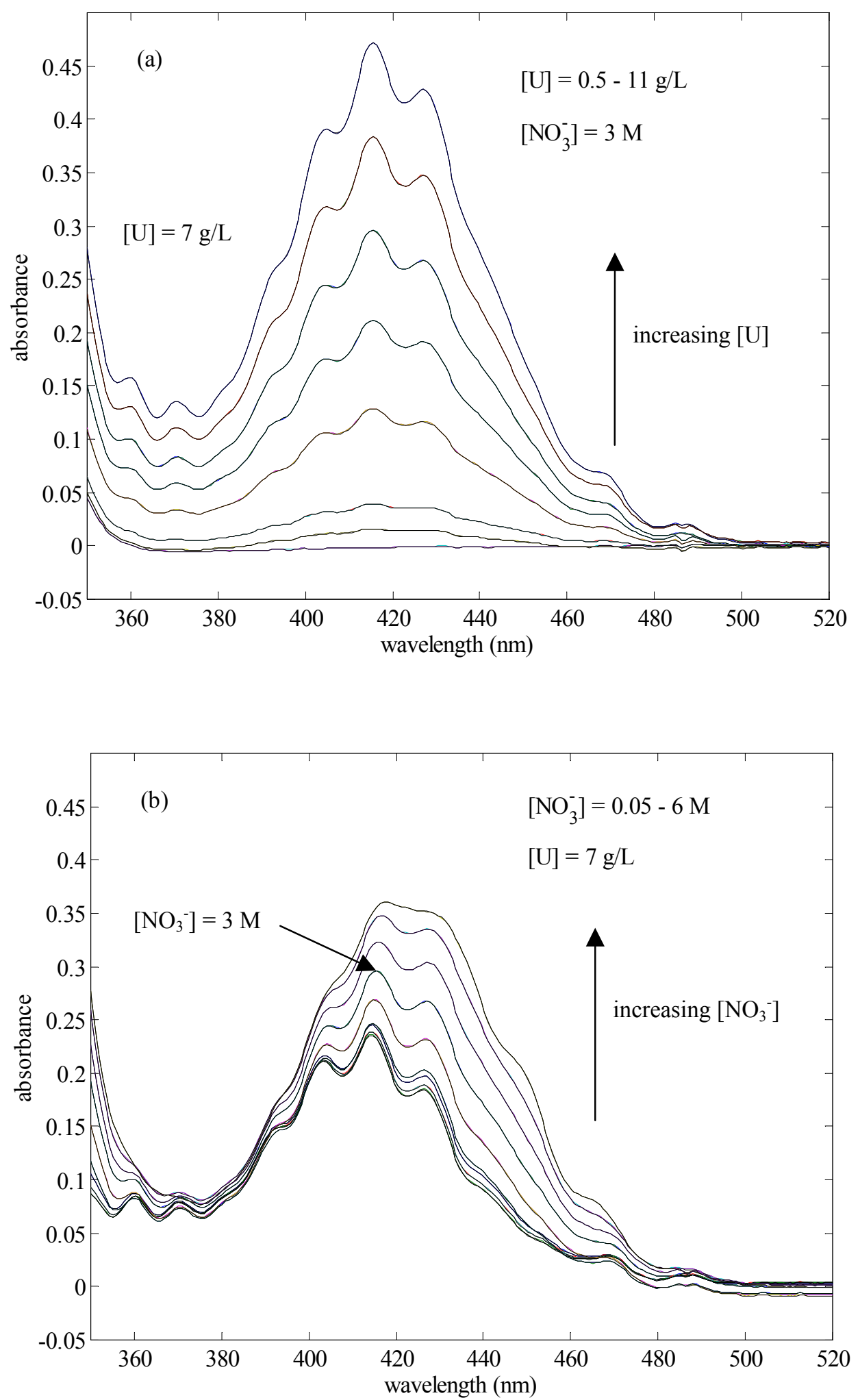

Figure 14. UV-visible absorption spectra of uranyl nitrate. 


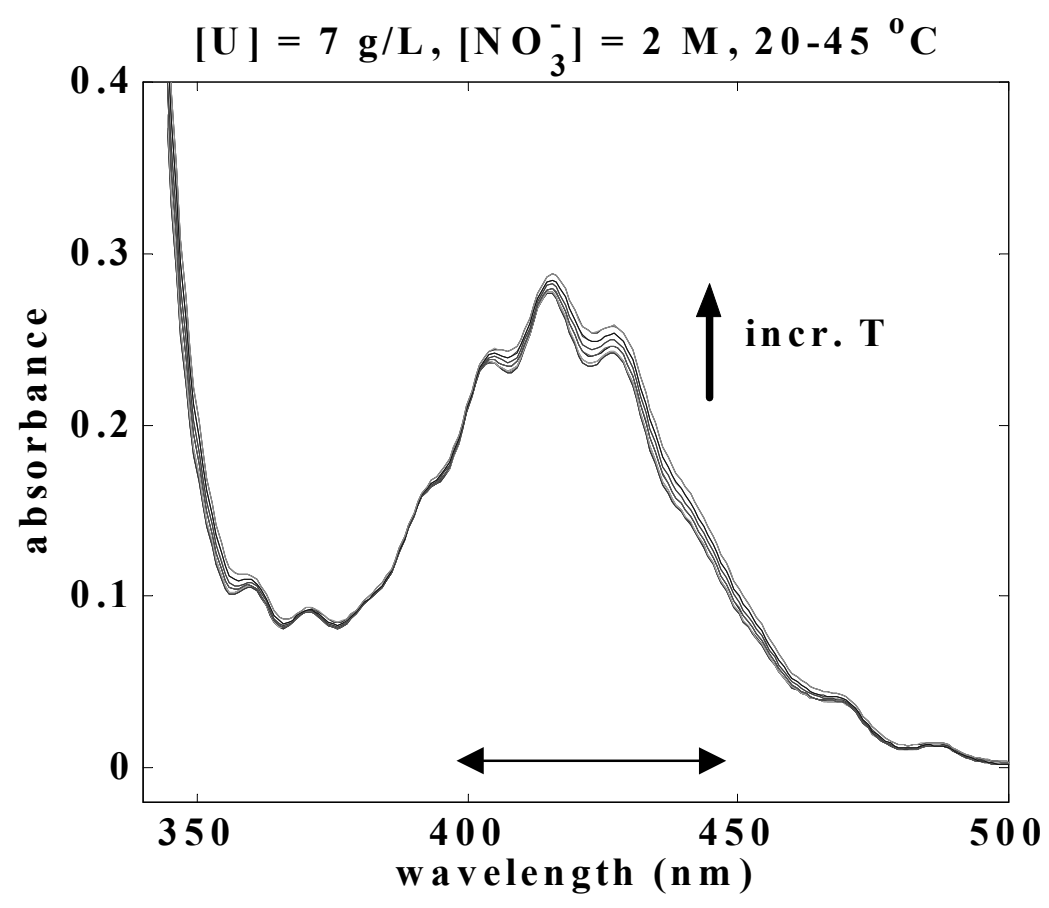

Figure 15. Effect of temperature on uranyl nitrate spectroscopy.

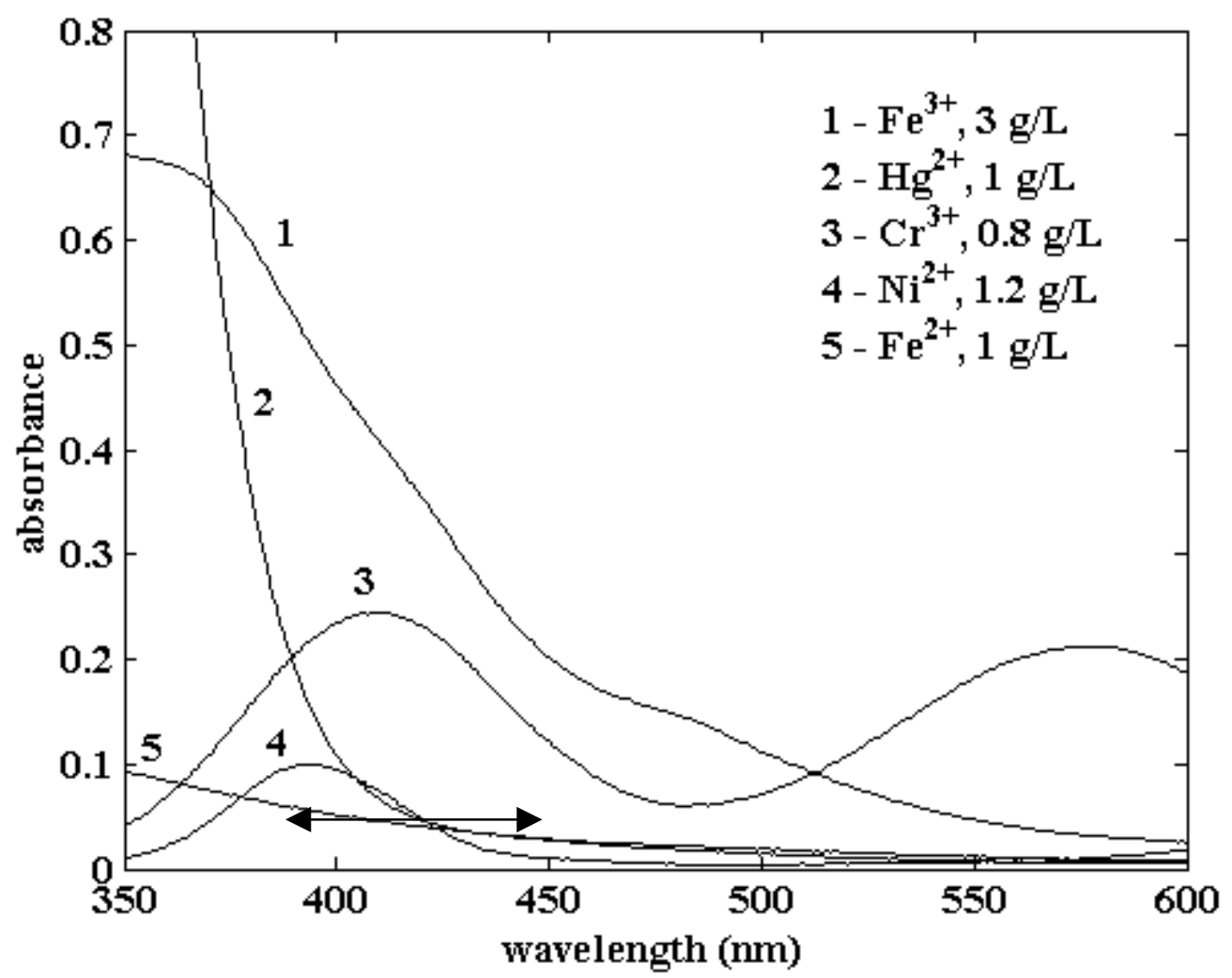

Figure 16. Spectra of typical interferents. 


$$
c=m \cdot \log \left(\left(I_{A}-I_{D}\right) /\left(I_{T}-I_{D}\right)\right)+b
$$

Here, $I_{A}$ and $I_{T}$ are the intensities of the absorbed and transmitted light, $I_{D}$ is the detector dark current (measured with the lamp off), and $m$ and $b$ are the "slope" and "zero" factors set during the calibration process. At Tanks 14.2 and 15.7, colorimetry is feasible because (a) nitrate concentrations are very low (less than $0.5 \mathrm{M}$ ), where the effects on uranyl spectroscopy are small; (b) the temperature variations are not large; and (c) there are low concentrations of iron and other interferents. Thus, none of the assumptions inherent to Beer's Law are violated. However, some or all of these conditions do not hold for the other tanks listed in Table 1, especially those $(12.2,12.3)$ that are upstream of the first set of mixer-settler banks. More information must therefore be drawn from the spectrum to make an accurate analysis at those points.

Chemometric data modeling, particularly Partial Least Squares (PLS) analysis, ${ }^{12,13}$ has proven to be an effective technique for extracting chemical information from data sets such as absorption spectra ${ }^{14,15}$. While "chemometrics" can involve any type of mathematical operation, including data derivatization, smoothing, and familiar statistical techniques such as linear regression, the term is generally understood to imply inverse multivariate calibration techniques. The term "multivariate" refers to the use of vectors of data, such as a spectrum or a collection of single-point data such as pressure, temperature, $\mathrm{pH}$, etc., which correlate in some way with the desired physical characteristic (i.e. concentration). The term "inverse" means these techniques determine that correlation by modeling the concentrations as a function of the data. This approach is the opposite of that implied by an equation such as Beer's Law, in which the spectra are presented as a function of the concentrations. However, it is consistent with the intended use of the instrument, specifically the prediction of concentrations after data collection. The value of the inverse approach comes from the fact that variations in the data which do not correspond to variations of concentration will not become part of the correlation. Thus, the effects of spectral noise, chemical interferents, temperature, etc., are greatly reduced. It is important to note that the extraneous effects must be present in the calibration set for the correlation to "learn" to ignore them.

A complete discussion of PLS is beyond the scope of this report, but can be found in the above references. However, some further explanation is desirable so that the calibration results can be understood. The entire set of calibration data is a matrix with $N$ spectra and $M$ wavelengths, with a vector of $N$ concentrations associated with the spectra. In PLS, the goal is to find a set of regression vectors ${ }^{*}$, each of length $M$, which, when multiplied by the calibration matrix, will reproduce the calibration concentrations. The first regression vector will be calculated to minimize the error between the true and predicted concentration vectors. The residual concentration vector is determined, and then the second regression vector is calculated to minimize the prediction error for the new concentration vector. The second vector will be orthogonal to the first within the $M$-dimensional space defined by the calibration spectra. This process is repeated until there are no longer

\footnotetext{
*Also called "latent vectors" (LVs) or "principal components".
} 
significant improvements in the concentration predictions. ${ }^{*}$ Generally, the number of regression vectors will be much smaller than $M$, indicating that the useful information content of the data has been extracted into a more efficient form. Importantly, the part of the data which does not correlate to concentration variations is mostly left behind. The regression vectors are the heart of the model which is used for data analysis; subsequent spectra are multiplied into each of the regression vectors, and the sum of those products is the predicted concentration.

There are other aspects of model building which critically affect the quality of the model. These include wavelength selection, data pretreatment (smoothing, derivatization, normalization, etc.), construction of the calibration set, and determining outliers, among others. Discussion of these factors as they apply to this specific application will be presented in Section 5.4.

\subsection{Experimental details}

The spectrophotometer system described in this report is comprised of two spectrophotometers operating in parallel. One instrument was installed in H Canyon in November, 1999, with troubleshooting, instrument refinement, and performance validation occurring over the next year and a half. The second instrument was installed in May, 2002, with troubleshooting and validation underway during the writing of this report. Unique calibration models were developed for each instrument. Although the same steps were taken in creating the models, some details such as stock solution concentrations, model parameters, calibration set size, etc., differed. Where possible, only data and figures which are representative of all calibrations will be presented, and details unique to each calibration will be presented only where necessary.

Solutions. Calibration solutions were made gravimetrically from stock solutions of uranium, nitric acid, and water. This is the most accurate method of making calibration solutions, as mass and density measurements can be made with high precision. A representative distribution of uranium and nitric acid concentrations for a calibration set is shown by the dots in Figure 17. The concentration ranges, $0-11 \mathrm{~g} / \mathrm{L} \mathrm{U}$ and $0.05-7 \mathrm{M}$ $\mathrm{NO}_{3}{ }^{-}$, span the expected concentrations of those species in the process tanks during normal and process upset conditions. Stock solution concentrations were determined by Analytical Development Section (ADS) standard analytical techniques. For these solutions, the uranium concentration uncertainty $(1 \sigma)$ is $1 \%$ (ICP-MS, 10 replicates) and the nitric acid uncertainty $(1 \sigma)$ is $2 \%$ (titration, typical method uncertainty). Uranium stock solutions contained $\sim 30 \mathrm{~g} / \mathrm{L} \mathrm{U}$ and $\sim 0.3 \mathrm{M}$ nitric acid. The nitric acid stock concentration was $\sim 6 \mathrm{M}$ for one solution set and $\sim 8 \mathrm{M}$ for the other set. Solutions masses were measured with balances calibrated under the SRTC Measurement \& Test Equipment program and/or the ADS Measurement Systems \& Equipment program. Balance uncertainties are typically $0.1 \%$. Solution densities were measured with hand-held densitometers that had

\footnotetext{
* There is no fixed algorithm for determining the precise number of regression vectors. The decision is based on the mean concentration residuals, structure of the regression vectors, and other diagnostics.
} 
been validated according to manufacturer instructions before and after the measurements. Density uncertainties are $\pm 0.001 \mathrm{~g} / \mathrm{mL}$.

The uncertainties of the calibration standards for uranium and nitric acid are calculated by propagating the uncertainty contributions from the stock solutions and instruments. The main source of error for both analytes is the uncertainty of the components in the stock solutions. For all calibration standards, uranium concentration uncertainty is $1.5 \%$, and nitric acid uncertainty is $2.2 \%$. These uncertainties will be used when calculating the total instrument uncertainty. Sample calculations are presented in Appendix 2.

Validation solutions came from several sources. Solutions used during model development were made by the same procedures as the calibration standards, and have the same uncertainties. Representative concentrations are displayed by the crosses in Figure 17. Other uranyl nitrate solutions, not represented in Figure 17, were mixed in the Warm Sample Aisle of H Canyon, and their concentrations were confirmed by 772-F Central Laboratories (CLAB). These solutions were poured into the samplers and held in the flow cell. Final validation occurred by comparing the spectrophotometer value obtained during sampler operation to CLAB analysis of the collected sample. The final validation provides the most rigorous test of instrument performance.

In the laboratory, solutions comprised of various metal ions and other potential interferents were mixed and analyzed. Solutions of $\mathrm{Fe}^{3+}, \mathrm{Hg}^{2+}, \mathrm{Cr}^{3+}, \mathrm{Ni}^{2+}, \mathrm{Mn}^{2+}, \mathrm{Cu}^{+}, \mathrm{Zn}^{2+}$ and $\mathrm{Al}^{3+}$ were made from nitrate salts. $\mathrm{Fe}^{2+}$ was introduced from a ferrous sulfamate solution. $\mathrm{Pb}^{2+}$ solution was made from lead chloride. Boric acid, tri-butylphosphate (TBP), and dibutylphosphate (DBP) were added from neat solutions.

Equipment. The spectrometers used to collect calibration and validation data are the same instruments installed in the facility. While there are several techniques for transferring a calibration from one instrument to another ${ }^{13,16}$, they tend to lead to a degradation in the prediction quality, due to the added effects of spectral noise from both instruments. The other components of the spectrophotometer, e.g. lamp (Equitech International, dual source deuterium/tungsten-halogen), computer (IBM ThinkPad), multiplexer (Terahertz Technologies), fiber optics (5 meter length), peristaltic pump (Ismatech), cuvette holder (SRTC), and flow cuvette (Hellma, $1 \mathrm{~cm}$ path length, $3 \mathrm{~mm}$ aperture), are not intrinsic to the calibration. Aspects of these components which differ from the field-installed equipment are noted in parentheses above. The cuvette holder is an aluminum block manufactured to similar dimensions as the Lexan block used for the reference and standard cuvettes in the field, with identical fiber optic collimating lenses. The block is wrapped in heating tape and insulated. The temperature of the block is monitored and manipulated by a programmable temperature controller and RTD probe (Digi-Sense). The pump is used to pull solutions into the cuvette, and then push the solutions out into a waste bottle. Use of the same fibers and cuvette for the reference and sample measurements eliminates the need for zero files (Section 4.4) which would otherwise be used to account for transmission differences in the optical path. 


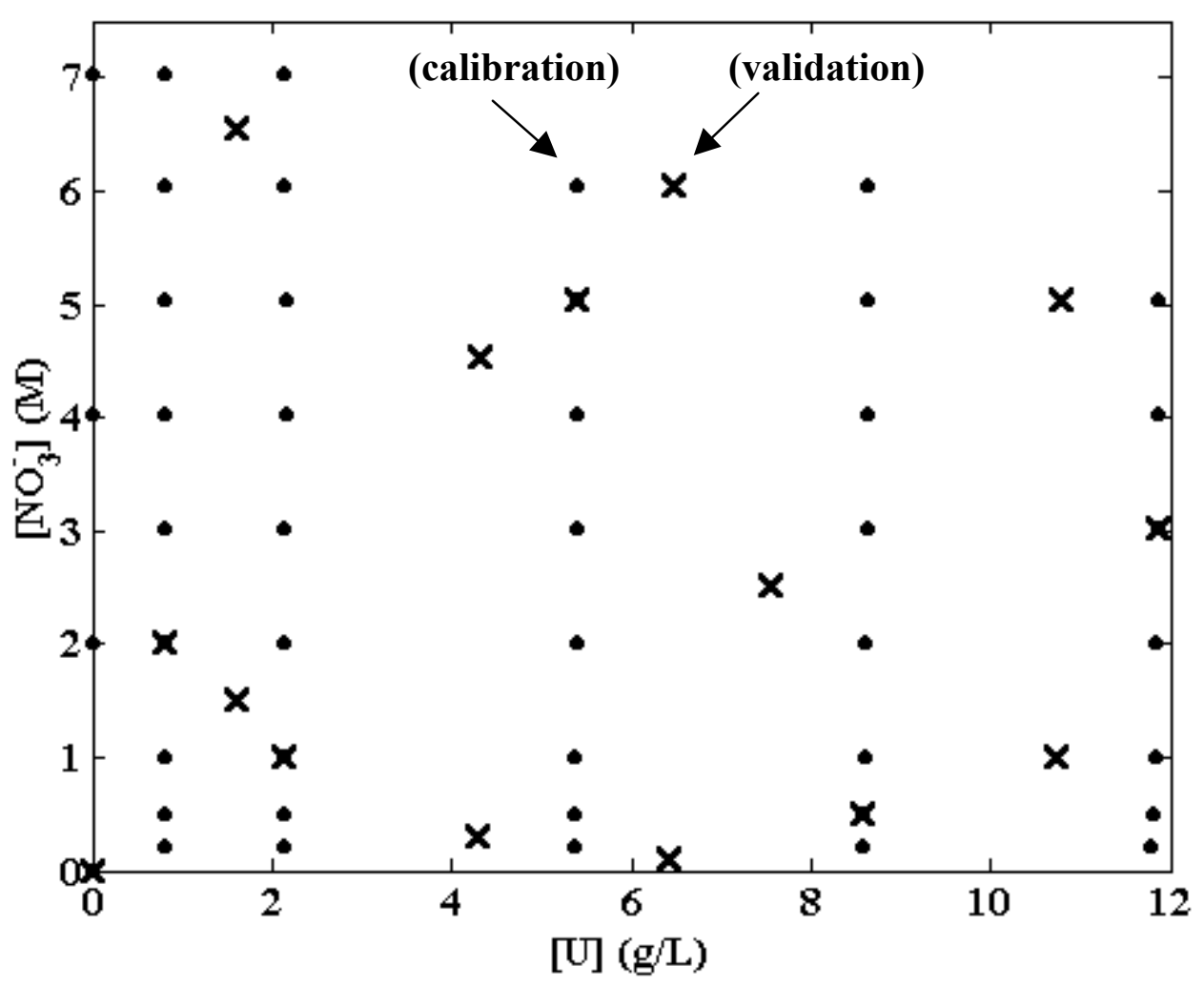

Figure 17. Distribution of calibration and validation set concentrations.

Absorbance spectra are obtained by calculating the ratio of the transmission of the solution in the flow cell to the transmission of a reference (distilled water) in the same flow cell. To ensure no sample cross-contamination, the flow cell is rinsed with an aliquot of the new sample before a second aliquot is measured. For spectra taken at elevated temperatures, the solution is allowed to equilibrate with the cuvette for 2 minutes. Data for the first calibration/validation set are taken at four temperatures between 20 and $50{ }^{\circ} \mathrm{C}$; data for the second set are taken at 20 and $50^{\circ} \mathrm{C}$ only, with an additional set of validation data taken at $35^{\circ} \mathrm{C}$. A new reference is obtained periodically to reduce baseline drift. Dark current is subtracted from each spectrum to remove constant detector noise levels.

Software. Model data acquisition was controlled by a program, zsfm.exe, written by ADS personnel using the Visual Basic platform. This program is a subset of the Onlinez.exe program using identical core data acquisition routines. The instrument configuration files, instru.dat and options.dat, contain the same information as their counterparts for the field version, except for the parts that relate to sampler operation and scheduling, which are not needed.

Scoping data analysis was performed in the Matlab 6.1 environment (Release 12, Mathworks, Inc., Natick, MA) using commercially available software (PLS_Toolbox 2.0, Eigenvector Research, Manson, WA). Matlab is also used to generate amended data sets incorporating spectral interferences (see below). Final models are generated using the 
ADS routine Mvasrs.exe. This program uses the same math routines present in $\mathrm{On}$ linez.exe, assuring identical performance between field and laboratory systems.

\subsection{Scoping studies}

As discussed in the Introduction, there are several chemical species which could be present in the HEU process and could interfere with the uranyl nitrate spectroscopy. The impurities listed in Table 2 largely arise from the fuel source cladding ( $\mathrm{Al})$ or catalyst $(\mathrm{Hg})$ added to the process. Stainless steel corrosion products (Fe, $\mathrm{Cr}, \mathrm{Ni}$, etc.) are present in the process. Tri-butylphosphate (TBP) and di-butylphosphate (DBP) could be entrained in the aqueous solutions during a process upset, and are otherwise present at low concentrations due to their limited (but finite) solubility in aqueous solutions. The possible effects of several species at concentrations characteristic of low-enriched uranium processing conditions, particularly boric acid and elevated concentrations of $\mathrm{Ni}, \mathrm{Cr}$, and $\mathrm{Mn}$, have been considered. Any other possible interfering species not explicitly mentioned in this report have not been studied. No inferences should be made concerning the ability of the instrument to provide accurate measurements in the presence of those species.

The first step in assessing potential spectroscopic interference is to establish which of these species have absorptions which overlap with those of uranyl nitrate. Spectra of potential interfering species are shown in Figure 16. Other species not shown, including $\mathrm{Al}, \mathrm{Mg}, \mathrm{Mn}, \mathrm{Na}, \mathrm{Pb}, \mathrm{Zn}$, TBP, DBP, and boric acid, do not have any absorptions in the relevant spectral regions. Of the species shown, the strongest absorptions will come from $\mathrm{Fe}^{2+}, \mathrm{Fe}^{3+}$ and $\mathrm{Hg}^{2+}$, which are present at the highest concentrations. $\mathrm{Cr}$ and $\mathrm{Ni}$ are at lower concentrations than are shown here and so the interferences due to these species are not expected to be as severe.

The effects of elevated solution ionic strength due to the presence of the interferents were determined by creating solutions with identical amounts of uranium and nitric acid, but different concentrations of each of the potential interferents. The spectrum of pure uranyl nitrate is subtracted from the spectrum of the uranyl nitrate plus interferent. If there is no effect due to ionic strength, then the effect of the interfering species will be linear, and the difference spectrum will be identical to the spectrum of the interferent. Species investigated include $\mathrm{Al}, \mathrm{Fe}, \mathrm{Cr}$, and $\mathrm{Ni}$. $\mathrm{Hg}$ was not examined due to issues surrounding the creation of mixed (radioactive and hazardous) waste. The results are provided in Figure 18. We demonstrate that the difference spectra and the pure interference spectra are similar for $\mathrm{Fe}, \mathrm{Cr}$, and $\mathrm{Ni}$. The variations that are observed can be attributed to small baseline shifts due to lamp drift and an aging reference spectrum. Derivatization of the spectra, which is required to account for the baseline offsets due to sampler operation, removes any of the differences seen here. The variations observed in the Al spectra cannot be attributed to the same lamp and reference spectrum causes. Nonetheless, the observed changes do not lead to differences in model predictions for either uranium or nitrate, as will be described in Section 5.4. Given the absence of an effect for Al, we can 

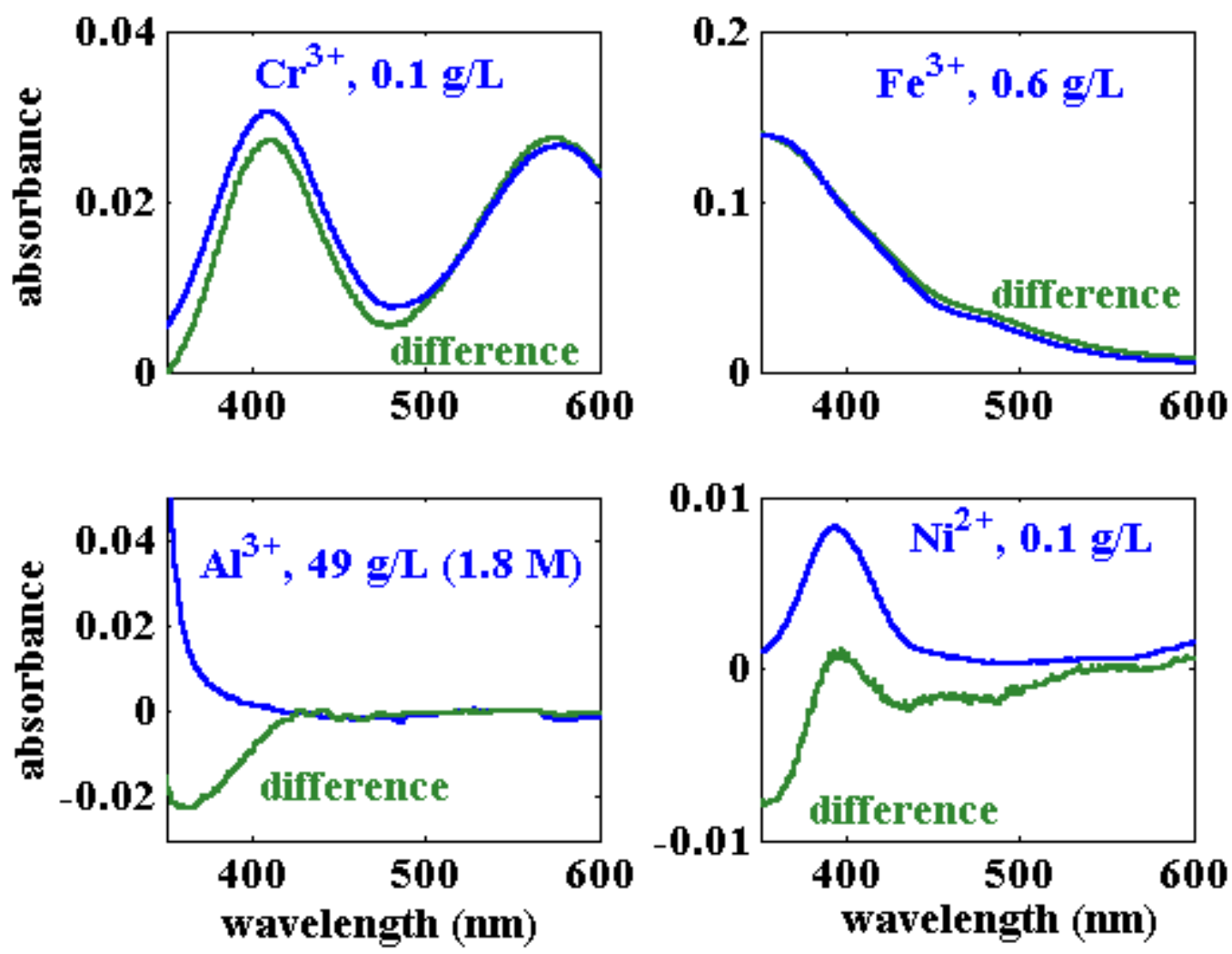

Figure 18. Effects of interferents on uranyl nitrate spectroscopy.

anticipate that other species such as $\mathrm{Na}$ and $\mathrm{Hg}$, which are present at lower concentrations and charge than the Al, will also have no effect on the spectra through changes in ionic strength.

The linear contributions of the interferences allow the simulation of their effects by mathematically adding the interference spectra to the uranyl nitrate spectra. Both calibration accuracy and time advantages are realized with this step. Accuracy of the calibration standards is preserved by reducing both the number of operations required to make the solutions and the cumulative uncertainty associated with each addition. Time is saved because mathematically manipulating the calibration solution spectra is quicker than making new solutions. The effects of increased interferent concentrations, or new combinations of interferents, can be determined quickly.

If interference effects are included in the calibration set, a chemometric analysis can be trained to ignore those effects. However, there are limits to how much interference can be accommodated in the calibration, as the effective signal-to-noise of the desired uranyl nitrate signal decreases with interference addition, and eventually the prediction capability of the calibration would degrade unacceptably. Thus, the choice of interferents incorporated into the calibration set should be restricted to those which, at concentrations expected in the field, have the most impact on the predictive abilities of both the uranium and nitrate models. To determine these interferents, PLS models for uranium and nitrate 
prediction were constructed using a calibration data set which did not include interfering species. The models were applied to spectra of a particular calibration solution $(7 \mathrm{~g} / \mathrm{L} \mathrm{U}$, $2 \mathrm{M} \mathrm{NO}_{3}{ }^{-}$) to which the spectra of various amounts of $\mathrm{Fe}^{2+}, \mathrm{Fe}^{3+}, \mathrm{Hg}^{2+}, \mathrm{Cr}^{3+}$, and $\mathrm{Ni}^{2+}$ had been added. $\quad$ The results, summarized in Table 3, indicate that at concentrations below expected process levels, $\mathrm{Fe}^{3+}$ has significant effects on both uranium and nitrate predictions. $\mathrm{Hg}^{2+}$ strongly affects uranium predictions at levels near the maximum expected value. $\mathrm{Fe}^{2+}$ affects both predictions at levels twice the expected concentrations. $\mathrm{Ni}^{2+}$ and $\mathrm{Cr}^{3+}$ levels listed here are larger than their expected concentrations (by 5, and $12.5 \mathrm{x}$, respectively), and generally will have less effect than the other species in the process. To minimize signal to noise degradation, we have included $\mathrm{Fe}^{3+}$ and $\mathrm{Hg}^{2+}$ in the calibration set, and will evaluate the resulting models to determine the effect of $\mathrm{Fe}^{2+}, \mathrm{Ni}^{2+}$, and $\mathrm{Cr}^{3+}$.

The effects of temperature were examined by creating prediction models for uranium and nitrate with data collected at one temperature $\left(20{ }^{\circ} \mathrm{C}\right)$ and using those models to predict concentrations of spectra collected at temperatures ranging from $20-50{ }^{\circ} \mathrm{C}$. Failure to include temperature variation in the calibration set has significant effects on both predictions, as shown in Figure 19. Clearly, temperature variation must also be included in the calibration set. Interestingly, prediction errors have a linear dependence on temperature, which suggests that data only needs to be taken at a few temperatures to obtain the desired corrections.

\subsection{Calibration}

Data sets. There are three pairs of uranium and nitrate models which were developed for the two spectrometers in this instrument. These model pairs, and the spectrometer and tanks to which they apply, are listed in Table 4. The first spectrometer has two pairs of uranium and nitrate models. The pair applied to Tanks 14.5, etc., do not incorporate the interferent corrections described above. The second pair, applied to Tank 12.2, and the pair used for the second spectrometer do incorporate those corrections.

Table 3. Effects of interferents on uranium and nitrate predictions.

\begin{tabular}{|c|c|c|c|}
\hline Species & Concentration $(\mathbf{g} / \mathbf{L})$ & Predicted $\mathbf{~}(\mathbf{g} / \mathbf{L})$ & Predicted $\mathbf{N O}_{\mathbf{3}}{ }^{-} \mathbf{( M )}$ \\
\hline None & --- & 7.07 & 2.12 \\
\hline $\mathrm{Fe}^{2+}$ & 2.0 & 5.10 & 5.16 \\
\hline $\mathrm{Fe}^{3+}$ & 0.2 & 3.60 & -6.4 \\
\hline $\mathrm{Hg}^{2+}$ & 1.0 & -0.10 & 2.15 \\
\hline $\mathrm{Ni}^{2+}$ & 0.5 & 6.55 & 1.71 \\
\hline $\mathrm{Cr}^{3+}$ & 0.2 & 8.18 & 2.16 \\
\hline
\end{tabular}

\footnotetext{
* The models were created in the Matlab environment using the same calibration data used for the initially installed spectrometer. The standard prediction errors are $0.12 \mathrm{~g} / \mathrm{L}$ for uranium and $0.10 \mathrm{M}$ for nitrate. Details of the fitting techniques are similar to those described below for the field-installed calibration models. Spectra of interferents matched to the wavelength calibration of the original spectrometer were obtained from spline fits of spectra taken on another spectrometer. This type of calibration transfer is acceptable for this study since the goal of the work is to remove the effects of the interferents, and not to predict their concentrations, which requires greater precision.
} 

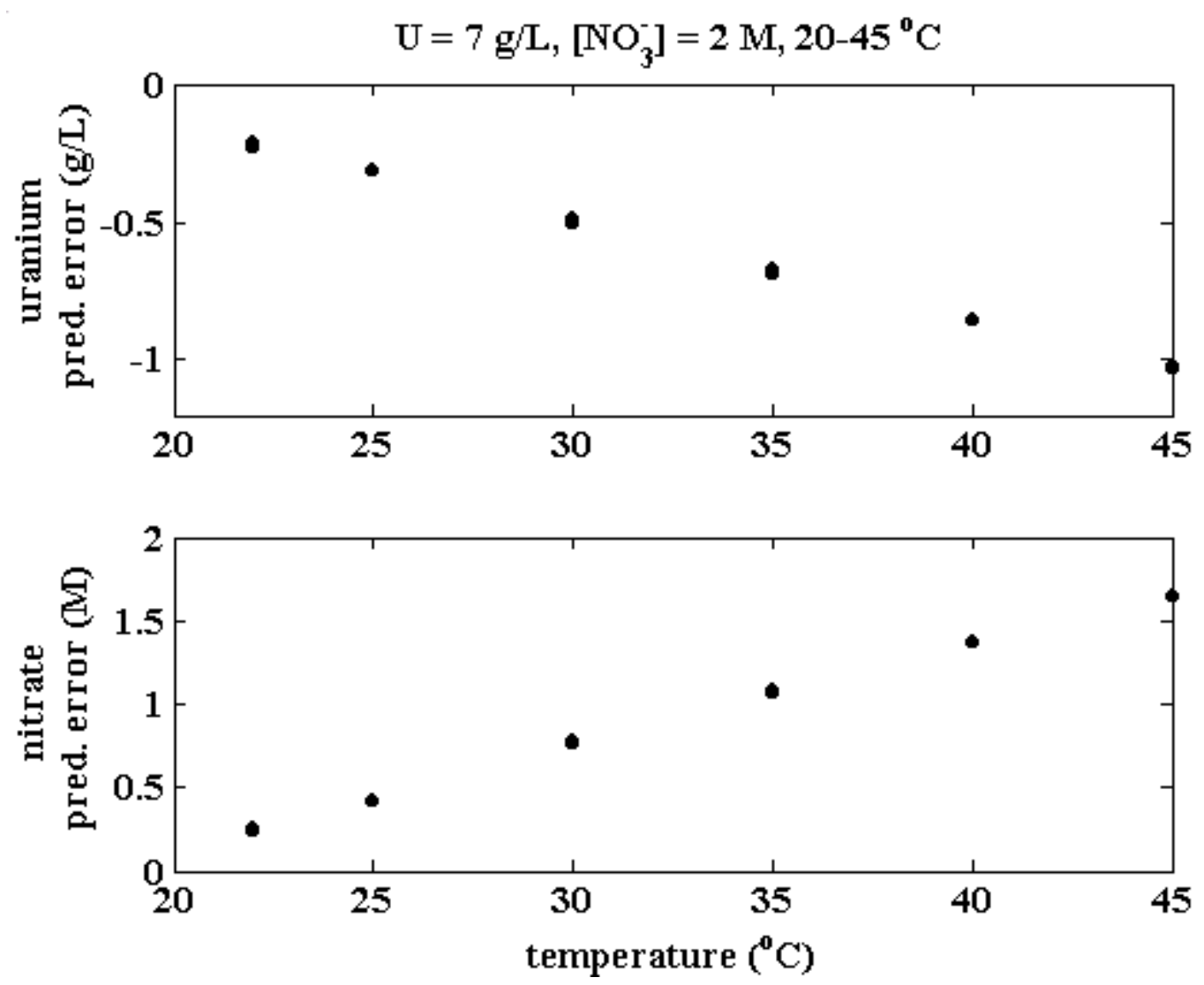

Figure 19. Temperature dependence of prediction errors for models created at 20 ${ }^{\mathbf{0}} \mathrm{C}$.

Table 4. Tank/spectrometer/model concordance.

\begin{tabular}{|c|l|l|l|}
\hline Spectrometer & \multicolumn{1}{|c|}{ Tanks } & \multicolumn{1}{|c|}{ Uranium } & Nitrate \\
\hline 1 & $15.4,16.8,17.5$, & HCURN03.PLS & HCNIT01.PLS \\
& 18.7 & & \\
\hline 1 & 12.2 & HCURN05.PLS & HCNIT04.PLS \\
\hline 2 & $12.3,14.5,17.4$, & HCURN21.PLS & HCNIT21.PLS \\
& $18.1,18.7$ & & \\
\hline
\end{tabular}

Generating a calibration model is an iterative process, in which the best set of fit parameters such as data treatment and outlier removal are determined from several trial models. There are slight differences in the development conditions for each calibration set. The differences do not lead to significant differences in the predictive ability of the models. For the first spectrometer models, spectra of each of the calibration solutions were obtained at $20,30,40$, and $50{ }^{\circ} \mathrm{C}$ in triplicate; for the second spectrometer, only data at 20 and $50{ }^{\circ} \mathrm{C}$ were obtained, based on the linear temperature dependence described above. For the models with interferences in the calibration, a randomly scaled amount of both the $\mathrm{Fe}^{3+}$ and $\mathrm{Hg}^{2+}$ spectra were added to each spectrum, with a maximum of $3 \mathrm{~g} / \mathrm{L}$ 
$\mathrm{Fe}^{3+}$ and $1 \mathrm{~g} / \mathrm{L} \mathrm{Hg}^{2+}$ added. Figure 20 shows the calibration set without and with the effects of random amounts of $\mathrm{Fe}^{3+}$ and $\mathrm{Hg}^{2+}$ (Figures 20(a) and (b), respectively). The distribution of interferent additions is shown in Figure 20(c). The spectral features due to uranyl nitrate are much less prominent in the new data set. Note that the spectra exceed 2 absorbance units at some wavelengths. While such an absorbance would normally lead to a deviation from Beer's Law, it is tolerable in this situation since the spectra are artificially constructed from data that follows linear behavior, and the models are constructed in regions that have somewhat less absorbance $(<1.5$ absorbance units). In the field, the absorbance for solutions containing these levels of $\mathrm{Fe}^{3+}$ and/or $\mathrm{Hg}^{2+}$ would be reduced by using a smaller path length. All models are generated using the Mvasrs.exe analysis program. The data are treated by taking the second derivative, which removes constant baseline offsets caused by sampler turbulence and sloping offsets caused by particulate scattering. The data are smoothed with a 9 or 11-point Gaussian function to reduce noise.

Uranium models. Uranium and nitrate models are generated independently. The best results for the uranium calibration were obtained with the data set truncated to 390-440 $\mathrm{nm}$. Outlier spectra were removed if the prediction error exceeded $4 \sigma$ for the calibration
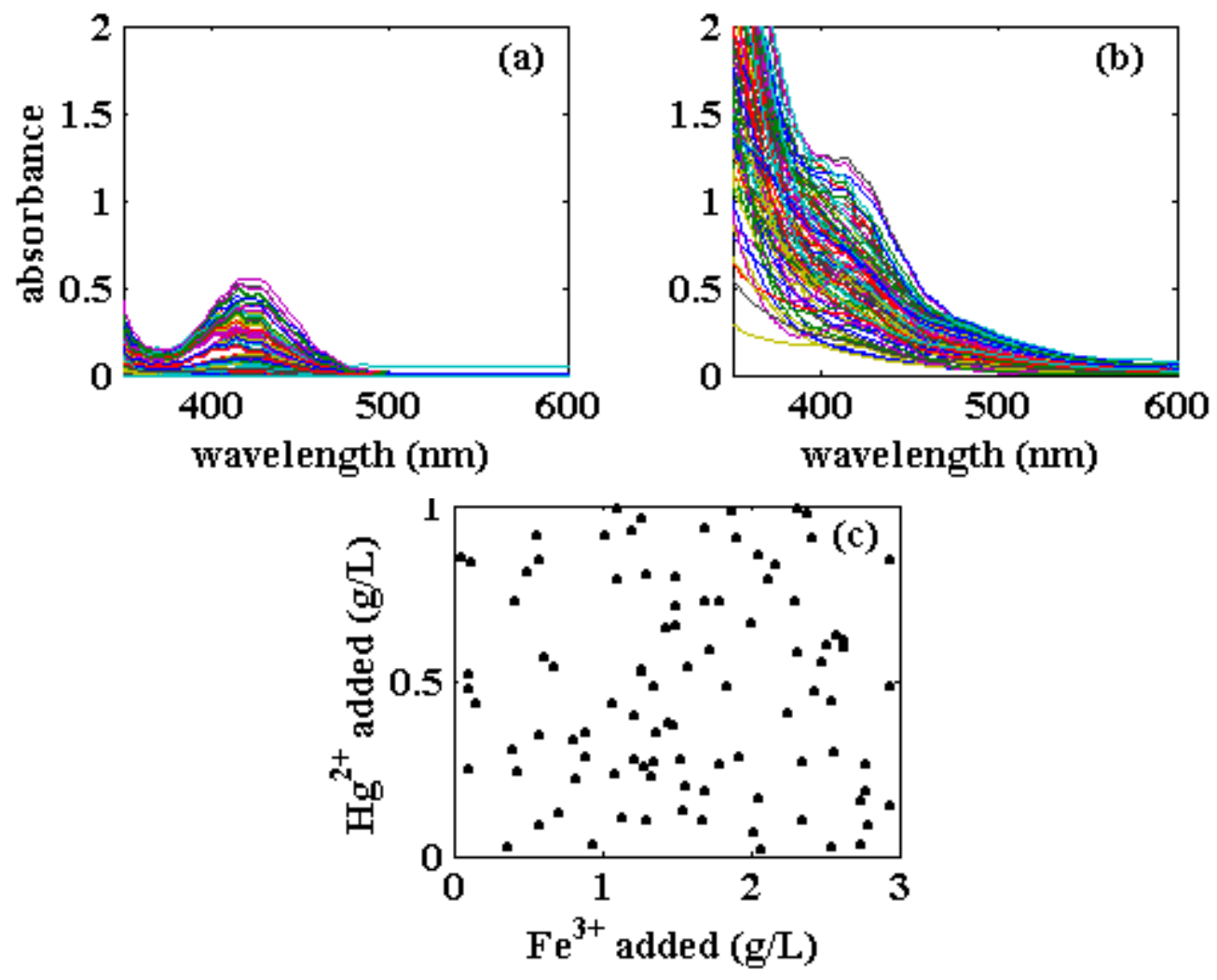

Figure 20. Effects of interferent spectra on calibration spectra. 
set taken as a whole. Less than $0.5 \%$ of the total number of spectra were removed as outliers, and their removal did not compress the concentration ranges shown in Figure 17. The most likely reason for spectra to become outliers comes from errors in data acquisition, especially if bubbles were undetected in the flow cell. Solutions which were sources for an outlier spectrum at one temperature often did not produce outliers at other temperatures, indicating that in those cases, sample prep error was the source of the error.

The results of a typical PLS analysis (in this case, for HCURN21.PLS) are shown in Table 5. These results indicate that for a model containing 6 regression vectors, the model self-prediction uncertainty $(1 \sigma)$ is $0.072 \mathrm{~g} / \mathrm{L}$. This value represents an average uncertainty for all samples. Prediction uncertainties for the other models are similar. The relatively high proportion of concentration variance predicted with the first regression vector ("latent vector") is consistent with the linear dependence of the spectra on uranium concentration at these levels. Additional regression vectors are required to account for the spectral changes due to nitrate and interferents. The $7^{\text {th }}$ and subsequent regression vectors have profiles that reflect instrument noise, and are rejected, as the instrument noise in the laboratory may not match that in the field, and the use of the additional vectors could degrade system performance. Although the concentration variance is well described, the spectral variance is not, and even subsequent regression vectors do not improve that measure. This behavior is consistent with the added noise due to the addition of the interferents. Figure 21 shows that the absolute uranium prediction accuracy does depend somewhat on uranium concentration (Figure 21(a)). This behavior will be discussed later in this section. The prediction accuracy is practically independent of nitrate concentration (Figure 21(b)). Inclusion of spectra from multiple temperatures removes all significant temperature dependence (Figure 21(c)). Recall that prediction errors of up to $1 \mathrm{~g} / \mathrm{L}$ could result from fits of spectra taken at $45{ }^{\circ} \mathrm{C}$ with models generated from spectra taken at $20{ }^{\circ} \mathrm{C}$. Thus, expected temperature variations for this application will not present a problem. Finally, note that the prediction error does not vary significantly with $\mathrm{Fe}^{3+}$ or

Table 5. Representative PLS results for uranium model.

\begin{tabular}{|c|c|c|c|}
\hline $\begin{array}{c}\text { Regression } \\
\text { Vector }\end{array}$ & $\begin{array}{c}\text { Spectral Variance } \\
\text { Explained } \\
(\boldsymbol{\%})\end{array}$ & $\begin{array}{c}\text { Concentration Variance } \\
\text { Explained } \\
(\%)\end{array}$ & $\begin{array}{c}\text { RMSECV } \\
\text { (g/L) }\end{array}$ \\
\hline 1 & 55.00 & 81.23 & 1.117 \\
\hline 2 & 67.09 & 90.83 & 0.546 \\
\hline 3 & 85.62 & 92.73 & 0.437 \\
\hline 4 & 87.03 & 93.98 & 0.363 \\
\hline 5 & 87.49 & 96.96 & 0.185 \\
\hline 6 & 87.94 & 98.82 & 0.072 \\
\hline 7 & 88.08 & 99.18 & 0.050 \\
\hline 8 & 88.09 & 99.28 & 0.044 \\
\hline 9 & 88.09 & 99.44 & 0.034 \\
\hline
\end{tabular}

a - RMSECV = Root-Mean-Square Error of Cross Validation. Equivalent to $\sigma$. 

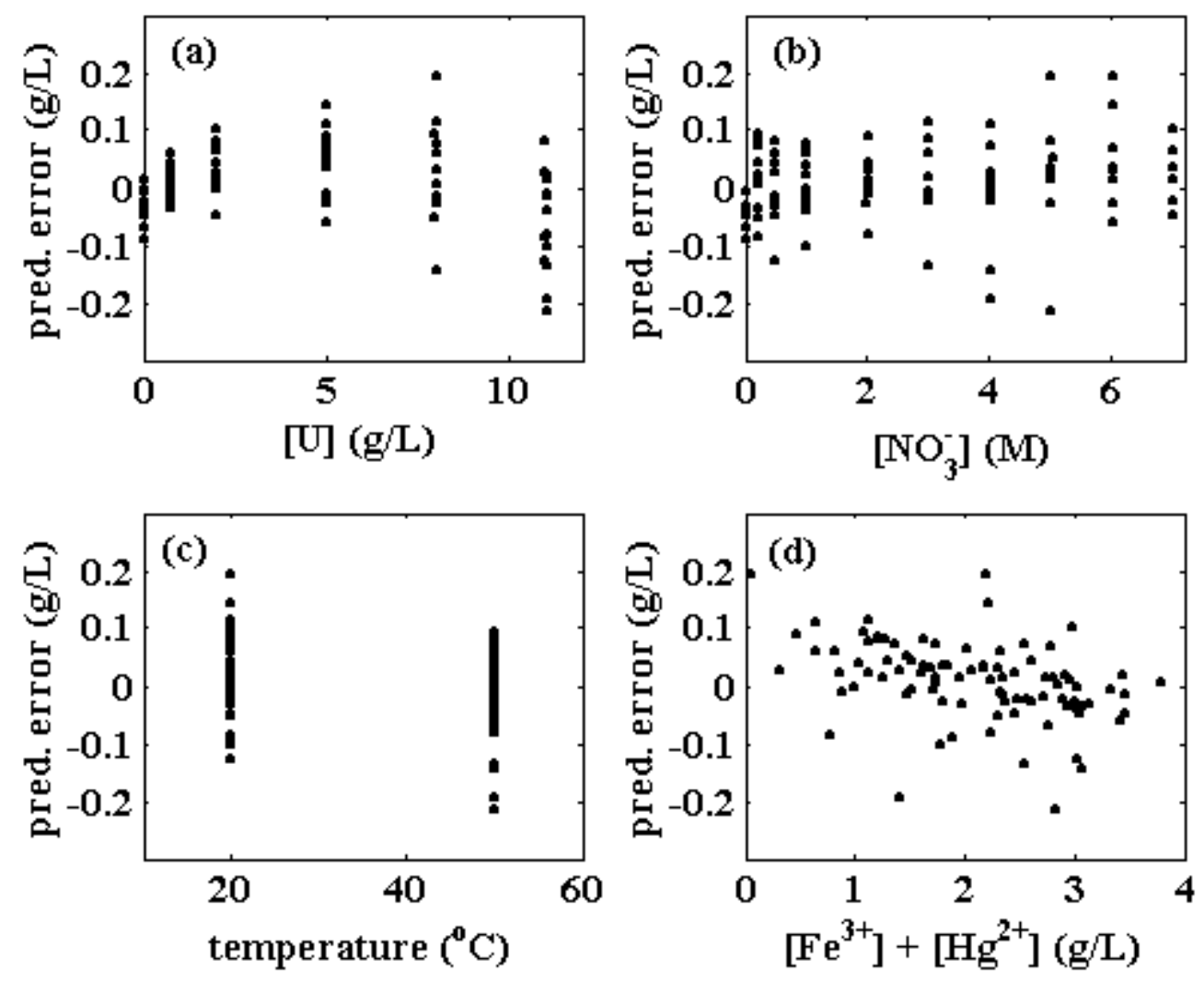

Figure 21. Typical uranium prediction error dependence on uranium and nitrate concentrations, temperature, and interferent concentration.

$\mathrm{Hg}^{2+}$ concentration (Figure 21(d)), which indicates that the model is effectively removing the contributions of these ions on the spectra. The latter result was confirmed by using the prediction model to fit several sets of spectra with the same uranyl nitrate content but different amounts of interferents, including one case with no interferents added. In all cases the fit uncertainties were almost identical to those of the original model.

The uncertainty (or root-mean-square error of cross-validation, RMSECV) used to describe the quality of the fit represents an average error for all samples. However, Figure 21(a) implies that there is a concentration dependence to the fit uncertainty. Figure 22 shows a concentration dependence of the prediction errors, expressed as a percentage of the concentration, for all three uranium models. As the dependence is consistent for all three uranium models, regardless of the inclusion of interferents, we have combined the points and fit them to a curve that roughly matches the distribution. ${ }^{*}$ Combining the points will also allow the calculation of a single uncertainty, independent of the model or spectrometer used to make the measurement. Expression of the uncertainty as a percen-

\footnotetext{
${ }^{*}$ The curve in Figure 22 is given by $(\%$ error $)=\left(-1.05 \times 10^{4}\right)\left(6.15 \times 10^{6}\right)^{-[\mathrm{U}]}+3 /[\mathrm{U}]+0.89$, where the parameters are chosen by a least-squares fit. It should be noted that the curve is designed to capture the shape of the distribution, and the form of the curve does not have a physical basis.
} 


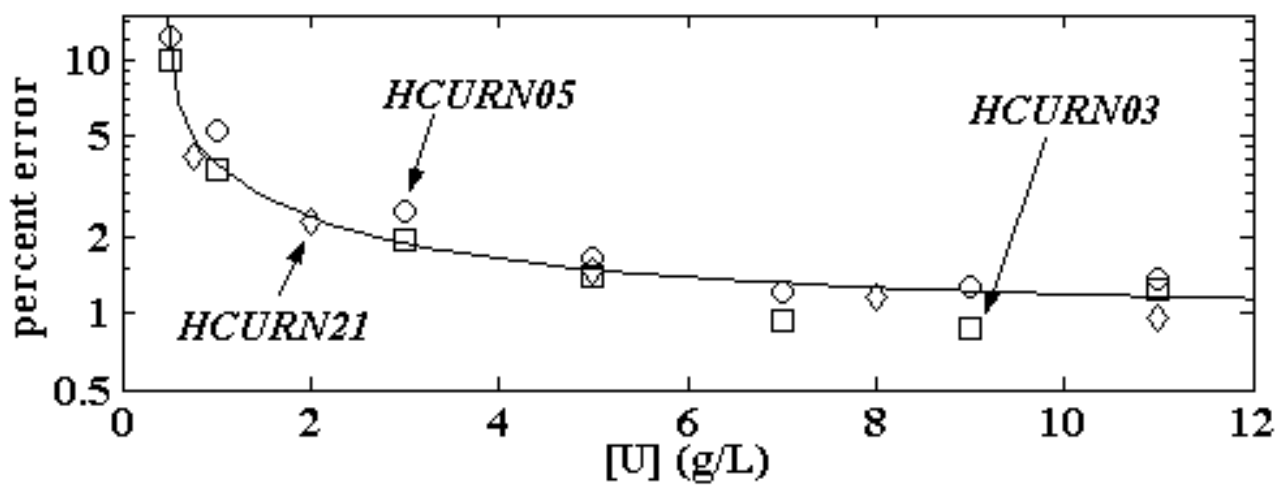

Figure 22. Dependence of uranium prediction uncertainty on uranium concentration for three models.

tage at this stage will make calculation of the total propagated uncertainty easier, since other contributions to the instrument error can also be expressed on a percentage basis.

Nitrate models. Prediction of the nitrate concentration requires additional data processing. The nitrate spectral dependence is not linear, as the distribution of uranyl nitrate complexes is not linear with nitrate concentration. ${ }^{9}$ Attempts to predict concentrations with PLS models fail to produce acceptable results. Table 6 shows that even with most of the spectral variance predicted, the concentration variance prediction is poor. In prior work, the situation was improved by normalizing the spectra to the maximum uranyl nitrate absorbance ${ }^{1}$ or the total integrated absorbance ${ }^{7}$. These approaches are not appropri-

Table 6. Representative PLS results for nitrate model.

\begin{tabular}{|l|l|l|l|l|l|l|}
\hline & \multicolumn{3}{|c|}{ Without normalization } & \multicolumn{3}{c|}{ With normalization } \\
\hline LV & $\begin{array}{l}\text { Spectral } \\
\text { variance } \\
\text { explained } \\
(\%)\end{array}$ & $\begin{array}{l}\text { Concentr } \\
\text { variance } \\
\text { explained } \\
(\%)\end{array}$ & $\begin{array}{l}\text { RMSECV } \\
(\mathbf{M})\end{array}$ & $\begin{array}{l}\text { Spectral } \\
\text { variance } \\
\text { explained } \\
(\%)\end{array}$ & $\begin{array}{l}\text { Concentr. } \\
\text { variance } \\
\text { explained } \\
(\%)\end{array}$ & $\begin{array}{l}\text { RMSECV } \\
(\mathbf{M})\end{array}$ \\
\hline 1 & 51.71 & 24.37 & 7.29 & 53.13 & 29.82 & 2.52 \\
\hline 2 & 66.05 & 44.13 & 4.06 & 66.32 & 56.16 & 1.59 \\
\hline 3 & 80.04 & 45.02 & 3.97 & 77.00 & 71.52 & 1.04 \\
\hline 4 & 82.64 & 46.97 & 3.78 & 82.01 & 87.75 & 0.46 \\
\hline 5 & 84.39 & 47.20 & 3.78 & 82.64 & 90.04 & 0.38 \\
\hline 6 & 84.62 & 47.86 & 3.74 & 82.75 & 96.30 & 0.14 \\
\hline 7 & 84.71 & 48.72 & 3.66 & 82.84 & 96.88 & 0.12 \\
\hline 8 & 84.72 & 50.87 & 3.38 & 82.85 & 97.30 & 0.10 \\
\hline 9 & 84.72 & 52.45 & 3.21 & 82.85 & 98.26 & 0.066 \\
\hline
\end{tabular}

a - RMSECV $=$ Root-Mean-Square Error of Cross Validation. Equivalent to $\sigma$. 
ate for on-line measurements. Both quantities will be altered by the presence of highly absorbing interferents, and are also difficult to calculate for second derivative spectra.

The purpose of the normalizations is to eliminate the dependence on uranium concentration from the spectra. As each of the species in the uranyl nitrate equilibrium has one uranium atom in it, the elimination can be done most directly by dividing the spectra by the uranium concentration determined from the uranium model. The efficacy of this approach is shown in Figure 23, which shows how the original spectra (Figure 23(a)) collapse into bands characteristic for each nitrate concentration (Figure 23(b)) after normalization. Thus, the analysis becomes an exercise in matching the different line shapes.

Nitrate models are derived from data obtained from 400-460 nm. The improved results with normalization is shown in Table 6 (for HCNIT21.PLS). All measures of fit quality are similar to those for the uranium models. On a percentage basis, the prediction accuracy is degraded somewhat, since the spectral changes are not linear with changes in nitrate concentration. A model with 6 regression vectors is the proper choice without introducing excessive instrumental noise. The self-prediction uncertainty $(1 \sigma)$ is $0.14 \mathrm{M}$. The diagnostic plots in Figure 24 show that prediction error does vary with nitrate concentration, there is little uranium dependence, and the temperature dependence is again muted (Figures 24(a-c)). As with the uranium fits, there is no apparent dependence on the amount of interferent added to calibration spectra (Figure 24(d)), and changes in the distribution of the $\mathrm{Fe}^{3+}$ and $\mathrm{Hg}^{2+}$ additions did not alter the accuracy of the fit.

The dependence of prediction uncertainty on nitrate concentration can be seen more clearly in Figure 25, in which the percentage error is plotted for all three nitrate models. The distribution of the concentration dependence is similar to that seen for the uranium
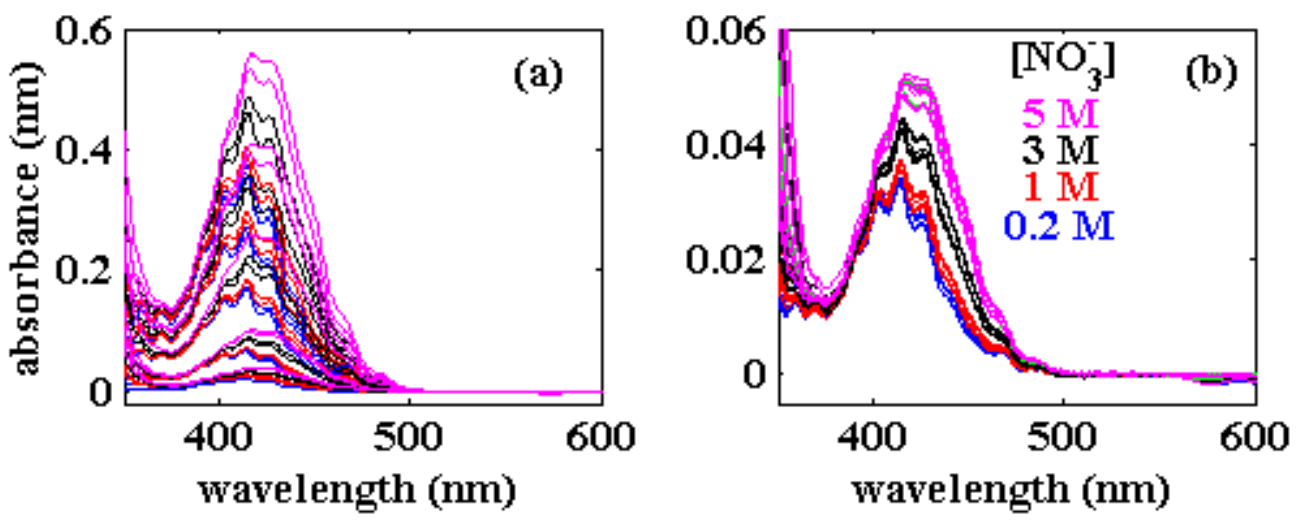

Figure 23. Effects of normalization by [U] on spectra.

\footnotetext{
* If the nitrate model name contains the string 'NIT', the data acquisition program will perform the normalization.
} 

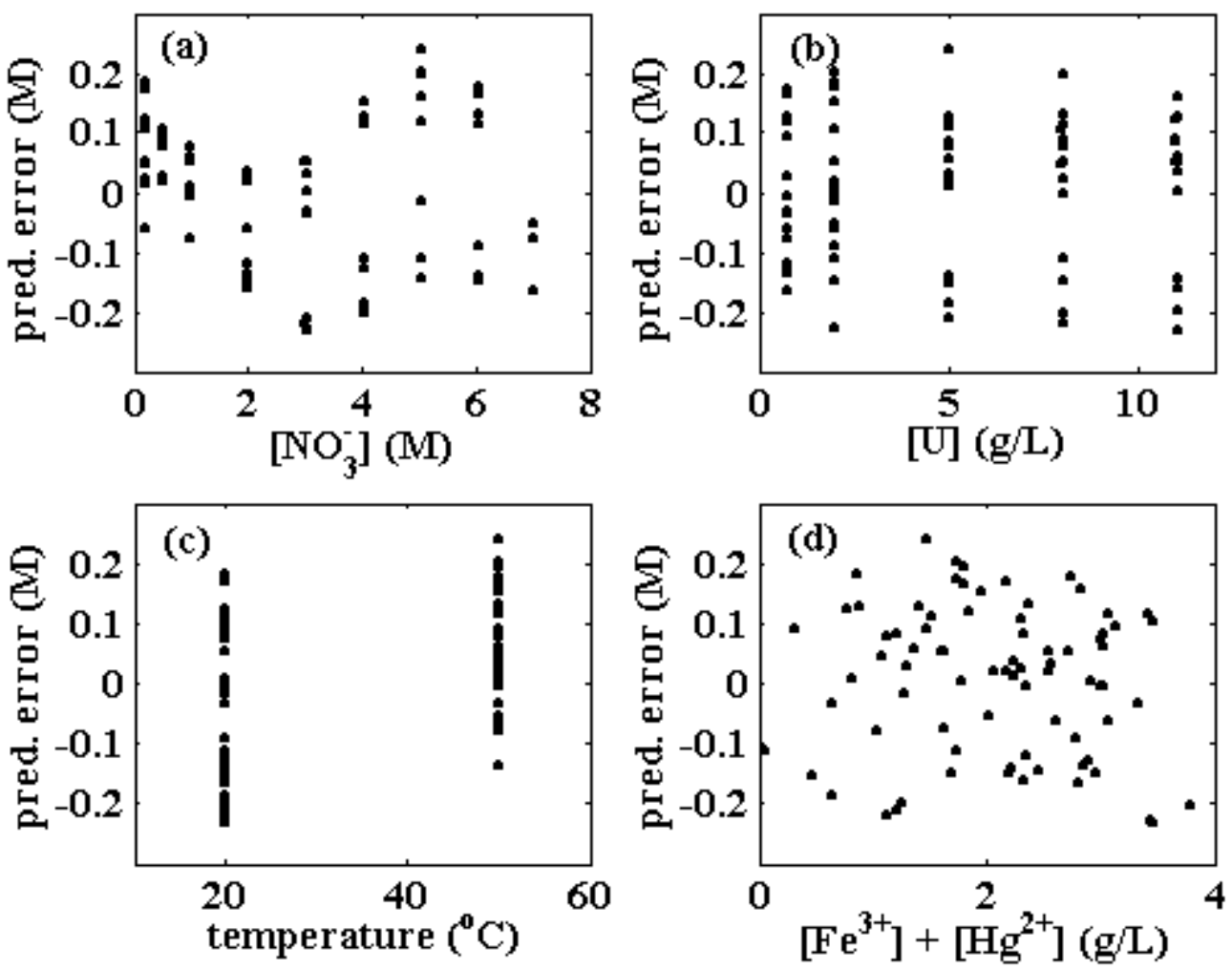

Figure 24. Typical nitrate prediction error dependence on uranium and nitrate concentrations, temperature, and interferent concentration.

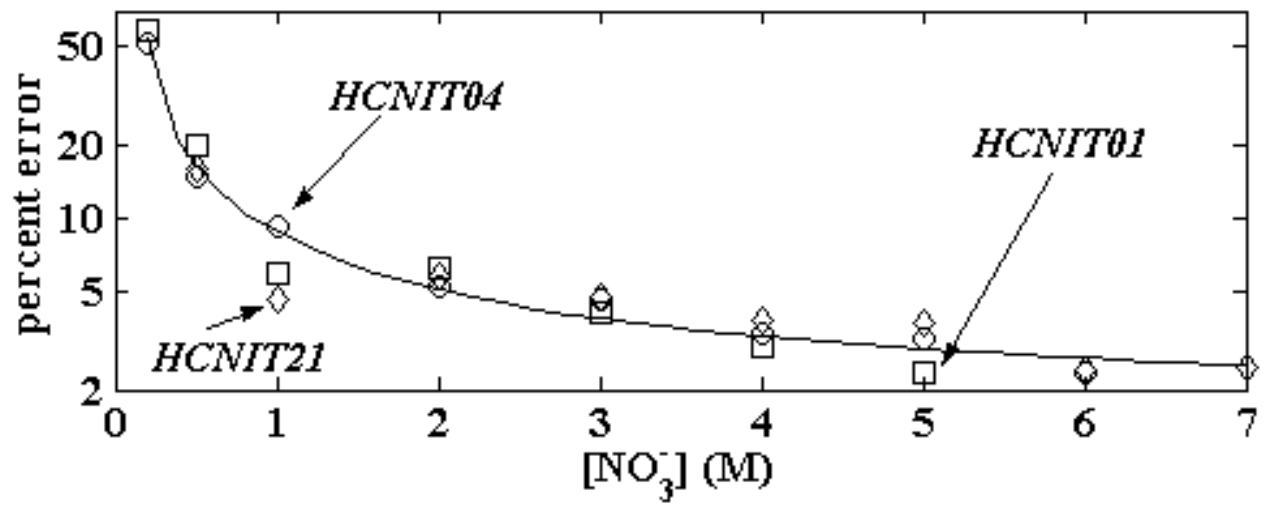

Figure 25. Dependence of nitrate prediction uncertainty on nitrate concentration for three models. 
models. The curve is a fit of an arbitrary function ${ }^{*}$ with no implied physical meaning to all data points, as described for the uranium analysis, and will be useful for calculating total instrument uncertainty.

Interferences. Inclusion of the effects of $\mathrm{Fe}^{3+}$ and $\mathrm{Hg}^{2+}$ in the calibration set has the benefit of improving the model performance for solutions containing other interferents such as $\mathrm{Fe}^{2+}, \mathrm{Ni}^{2+}$, and $\mathrm{Cr}^{3+}$. Table 7 demonstrates the improvements for the solution previously described in Table 3. In all cases where there were significant prediction errors, the errors have been greatly improved or eliminated in the new model. The result is not completely unexpected, as the absorbances of these species have similar shapes, in that they tend to increase at lower wavelengths. Thus the corrections incorporated into the models to account for $\mathrm{Fe}^{3+}$ and $\mathrm{Hg}^{2+}$ should provide some benefit for the other species. $\mathrm{Cr}^{3+}$ does have a different shape, with a maximum near the uranyl nitrate absorbance peak. It also produces the largest prediction errors. However, the deviations caused by the interferents scale linearly with their concentration, and as the $\mathrm{Cr}^{3+}$ concentration represented here is 12.5 times larger than the highest level expected, $\mathrm{Cr}^{3+}$ should not be expected to alter the instrument results. Similarly, if $\mathrm{Ni}^{2+}$ is scaled to its maximum expected concentrations, it will also cause acceptably small deviations.

It is apparent that $\mathrm{Fe}^{2+}$ at its maximum expected concentration (half of the value shown in Table 7) may cause a small bias in both uranium and nitrate concentrations. The maximum predicted biases are $-0.25 \mathrm{~g} / \mathrm{L}$ for uranium and $-0.35 \mathrm{M}$ for nitrate. These values are independent of the uranium and nitrate concentrations, and are linearly dependent on $\mathrm{Fe}^{2+}$ concentration. The biases are at or slightly above the total instrument uncertainties (described below). The facility validation of the interferent-containing models will include evaluation of model predictions with tank samples which would include $\mathrm{Fe}^{2+}$. The observation of prediction biases at that stage of validation would suggest a recalculation of the models including the effects of $\mathrm{Fe}^{2+}$.

The effect of $\mathrm{Al}^{3+}$ on uranium and nitrate predictions using the interferent-containing models is shown in Table 8. Negligible differences are observed with uranium predictions. Nitrate predictions only vary within the model uncertainties, and there does not

Table 7. Effects of interferents on uranium and nitrate predictions.

\begin{tabular}{|c|c|c|c|c|c|}
\hline Species & Conc. $(g / L)$ & \multicolumn{2}{|c|}{ Predicted U (g/L) } & \multicolumn{2}{c|}{ Predicted NO ${ }_{3}^{-}$(M) } \\
\hline None & --- & \multicolumn{2}{|c|}{7.07} & \multicolumn{2}{c|}{2.12} \\
\hline & & No Fe/Hg & With Fe/Hg & No Fe/Hg & With Fe/Hg \\
\hline $\mathrm{Fe}^{2+}$ & 2.0 & 5.10 & 6.57 & 5.16 & 1.42 \\
\hline $\mathrm{Fe}^{3+}$ & 0.2 & 3.60 & 6.88 & -6.4 & 1.94 \\
\hline $\mathrm{Hg}^{2+}$ & 1.0 & -0.10 & 7.13 & 2.15 & 2.17 \\
\hline $\mathrm{Ni}^{2+}$ & 0.5 & 6.55 & 7.18 & 1.71 & 1.71 \\
\hline $\mathrm{Cr}^{3+}$ & 0.2 & 8.18 & 7.75 & 2.16 & 2.27 \\
\hline
\end{tabular}

a $\mathrm{No} \mathrm{Fe}^{3+}$ or $\mathrm{Hg}^{2+}$ interferences included in the model.

\footnotetext{
${ }^{*}$ The curve in Figure M12 is given by $(\%$ error $)=(-379.2)\left(6.15 \times 10^{6}\right)^{-[\text {nitrate] }}+7.25 /[$ nitrate $]+1.48$, where the parameters have been determined by a least-squares fit.
} 
Table 8. Effects of aluminum on uranium and nitrate predictions.

\begin{tabular}{|c|c|c|c|c|}
\hline Al con. $(\mathbf{M})$ & \multicolumn{2}{|c|}{ Predicted U (g/L) } & \multicolumn{2}{c|}{ Predicted $\mathbf{N O}_{\mathbf{3}}^{-} \mathbf{( M )}$} \\
\hline--- & No Al & With Al & No Al & With Al \\
\hline 0.9 & 2.05 & 2.05 & 3.05 & 2.83 \\
\hline 1.64 & 2.02 & 2.07 & 6.08 & 6.27 \\
\hline 1.8 & 2.05 & 2.05 & 5.34 & 5.46 \\
\hline
\end{tabular}

seem to be a correlation between $\mathrm{Al}$ concentration and change in nitrate prediction. Any correlation could reasonably be expected to be monotonic, which is not reflected in the data. Thus, the spectral differences due to changes in solution ionic strength caused by large quantities of $\mathrm{Al}^{3+}$ compared to pure uranyl nitrate solutions are not observed to lead to inaccuracies in predictions of uranium or nitrate concentrations.

Validation. There are several different levels of model validation which can occur, depending on the degree to which the sample preparation and data collection are removed from the conditions under which the calibration data were generated. The least stringentvalidation occurs with the analysis of solutions which are made with the same stock solutions as the calibration standards and are analyzed at the same time. An intermediate level is the analysis of independently mixed solutions held in the sampler flow cell in the field. The most stringent validation, which matches the ultimate use of the instrument, is sample analysis with the sampler operating. All three validation levels have been conducted for models HCURN03.PLS and HCNIT01.PLS on Tanks 14.5, 16.8, and 17.5. At this writing, only laboratory validations at SRTC have been conducted on the other models.

The laboratory validation data sets contained spectra of solutions which were not part of the calibration standard set, as well as spectra of both calibration and validation solutions taken at different temperatures than those used in the original calibration. Where appropriate, $\mathrm{Fe}^{3+}$ and $\mathrm{Hg}^{2+}$ spectral interferences were added to the validation sets. The laboratory results for the three model pairs (uranium and nitrate) are summarized in Table 9. In all cases, the prediction accuracies for the validation sets are consistent with the calibration uncertainties. The dependence of the validation set predictions on temperature is also consistent with the small observed dependences shown in Figures 21(c) and 24(c). Note that the uncertainties reported in Table 9 do not represent the total reportable uncertainty for the spectrophotometers, as the contributions of standard uncertainty and field operation (i.e. turbulence in the sampler) must also be considered.

Table 9. Calibration and validation results for models.

\begin{tabular}{|c|c|c|c|c|c|}
\hline Uranium & $\sigma_{\text {cal }}(\mathbf{g} / \mathbf{L})$ & $\sigma_{\text {val }}(\mathbf{g} / \mathbf{L})$ & Nitrate & $\sigma_{\text {cal }}(\mathbf{M})$ & $\sigma_{\text {val }}(\mathbf{M})$ \\
\hline HCURN03.PLS & 0.070 & 0.078 & HCNIT01.PLS & 0.101 & 0.078 \\
\hline HCURN05.PLS & 0.096 & 0.111 & HCNIT04.PLS & 0.120 & 0.093 \\
\hline HCURN21.PLS & 0.072 & 0.111 & HCNIT21.PLS & 0.138 & 0.145 \\
\hline
\end{tabular}


Representative intermediate and final validation data, including comparisons with CLAB analysis by diode array spectroscopy (DAS), for measurements at Tanks 14.5, 16.8, and 17.5 are presented in Table 10. The uranium and nitrate models predict accurately at each sampling point, showing that the point-specific corrections, manifested in the zero\#\#.mva data files, eliminate the effects of different optical paths (both fiber optics and flow cell) on the spectra. The data shown here were obtained over a 16-month period, during which the equipment and models have not been changed and have provided consistent results. Over time, small changes in the background spectra were observed. The changes are thought to arise primarily from displacement of the fiber optics. These changes were removed during spectral derivatization, and they did not affec the analysis results for either uranium or nitrate.

\subsection{Uncertainties}

Influence of sampler operation. Variations of the uranium and nitrate predictions can arise from operation of the sampler. The contributions of the sampler to the overall uncertainty can be determined by comparing the signal variation from analysis of the standard cell to that from a process sampler. This comparison allows all other instrumental factors to remain constant. The test assumes that there is no change in the contents of the tank being sampled. Results, for both uranium and nitrate, for the Tank 16.8 sampler are shown in Figure 26. Note that the sampler is not usually run for periods longer than a couple of days; hence there is no extended data as there is for the cuvette, which is monitored continuously. Nonetheless, one can see that the overall standard deviation and the peak-to-peak fluctuations in the sampler can be up to twice as large as the values for the cuvette. The standard deviations for the cuvette are consistent with those observed for replicate samples in the calibration sets, which are incorporated into the model uncertainties. Thus, the true sampler uncertainty must be calculated from the values noted in Figure 26. Assuming that the instrumentation $\left(\sigma_{\text {in }}\right)$ and sampler $\left(\sigma_{\text {sampler }}\right)$ uncertainties are independent, the total test uncertainty $\left(\sigma_{\text {test }}\right)$ can be calculated as

$$
\sigma_{\text {test }}^{2}=\sigma_{\text {in }}^{2}+\sigma_{\text {sampler }}^{2}
$$

For uranium, $\sigma_{\text {test }}=0.038 \mathrm{~g} / \mathrm{L}, \sigma_{\text {in }}=0.024 \mathrm{~g} / \mathrm{L}$, and thus $\sigma_{\text {sampler }}=0.029 \mathrm{~g} / \mathrm{L}$. For nitrate, $\sigma_{\text {test }}=0.057 \mathrm{M}, \sigma_{\text {in }}=0.030 \mathrm{M}$, and thus $\sigma_{\text {sampler }}=0.048 \mathrm{M}$. While the standard deviations for uranium and nitrate for sampler operation are smaller than the intrinsic model uncertainties, they are still appreciable and must be included in the total spectrophotometer uncertainty.

The operation uncertainty can be expected to vary between samplers, and even for a given sampler with changes of the jet pressure and leak rate. In fact, lower uncertainties have been observed for the Tank 14.5 and 17.5 samplers. At this writing there is insufficient data to determine if the sampler contribution to the uncertainty is an absolute value, or if, like the standard and model uncertainties described above, it can be expressed as a percentage of the derived value. It is assumed that this contribution is absolute, and that 
Table 10. Field validation results for Tanks 14.5, 16.8, and 17.5.

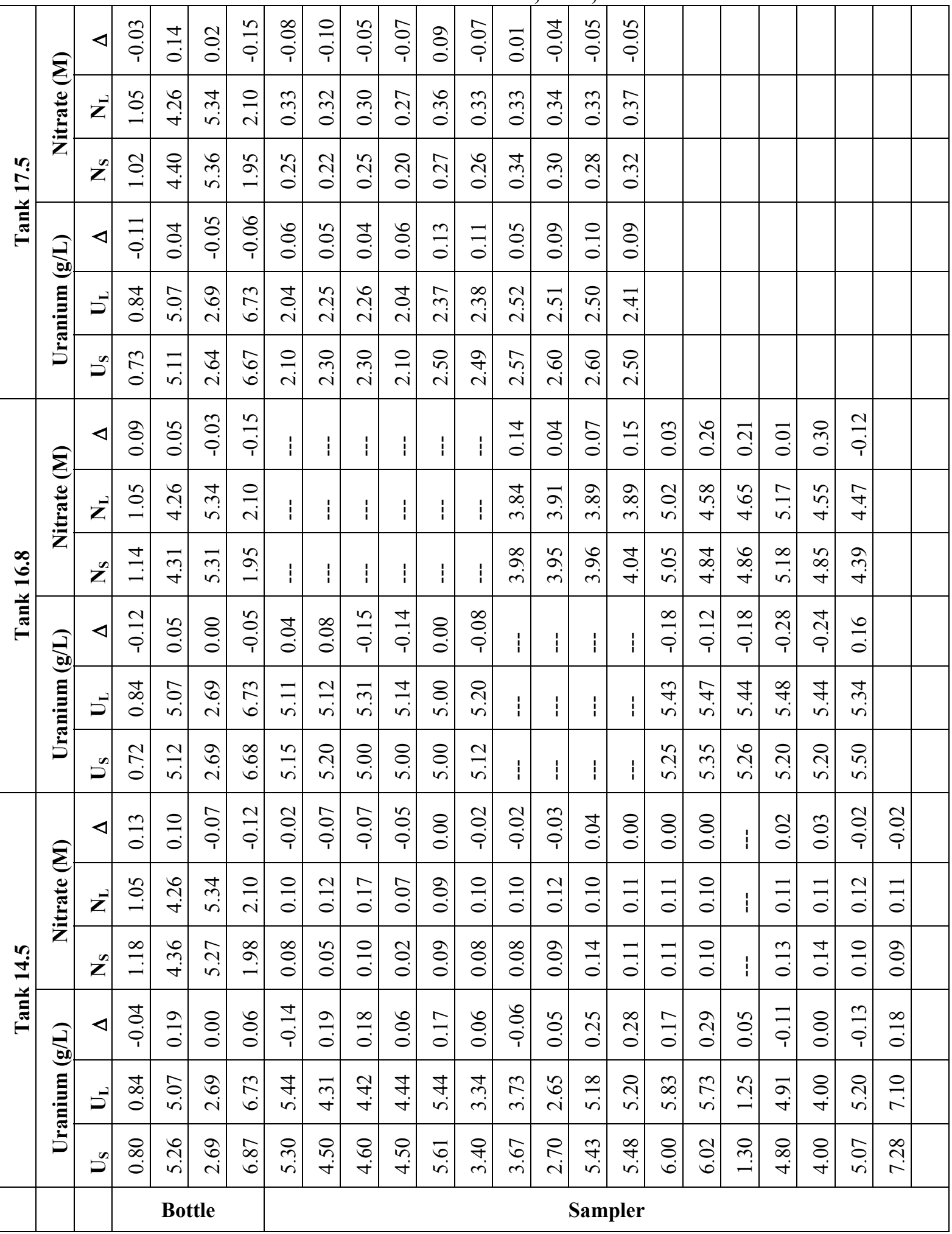

Uranium and nitrate analyses reported on the same row for a given tank represent simultaneous analysis of a single solution for both components. "S" and " $\mathrm{L}$ " subscripts refer to spectrophotometer and CLAB laboratory results, respectively; " $\Delta$ " is the difference between those two measurements. 

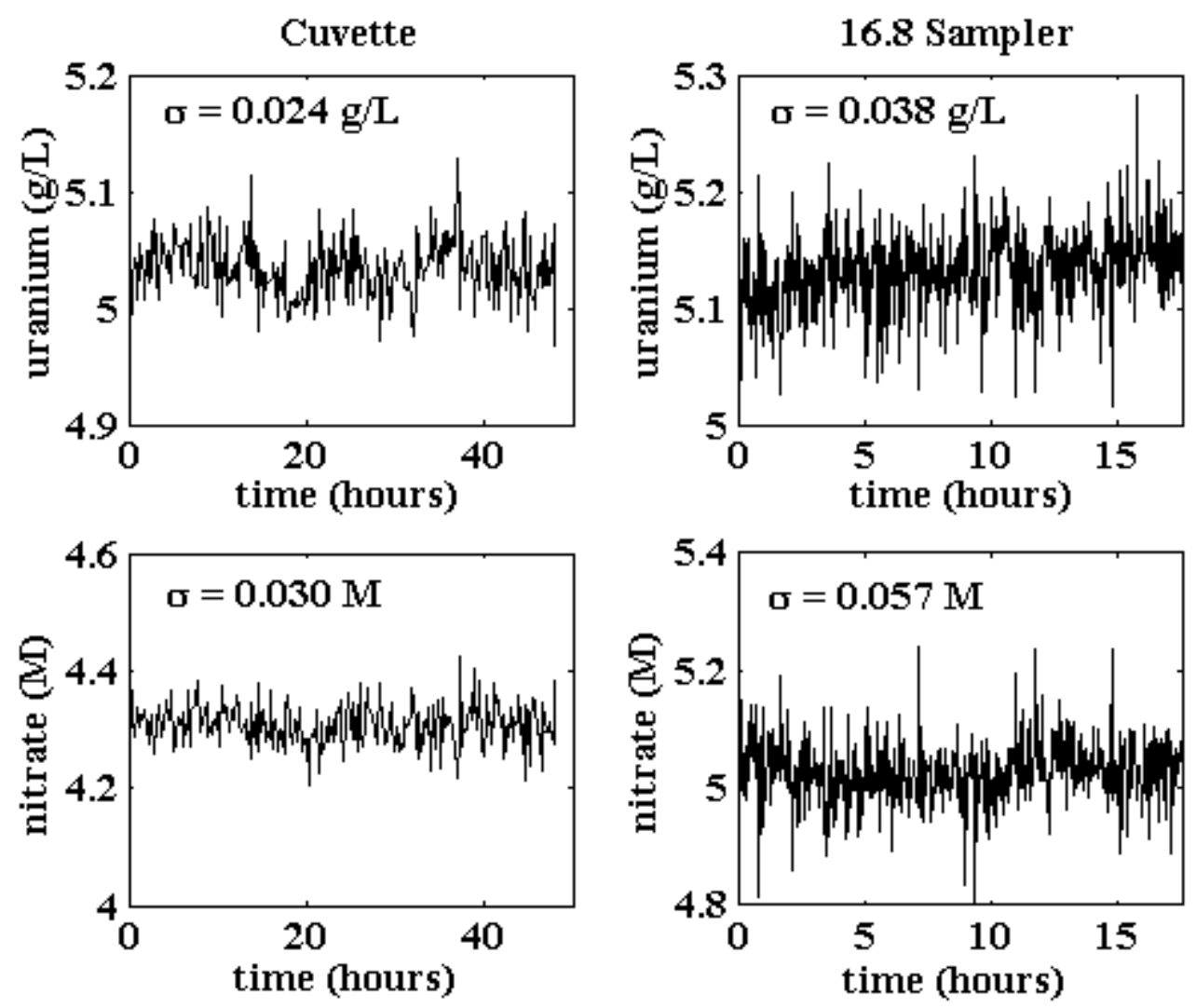

Figure 26. Comparison of standard cuvette and sampler uncertainties.

the example presented above describes typical behavior. The values derived here will be used in the calculation of the total instrument uncertainty, which is presented below.

Note that while sampler operation increases the uncertainty of a single analysis, it does not influence the mean of a series of analyses. This point is demonstrated in Figure 27, in which analysis results for Tank 16.8 are compared to the associated baseline offset, which is a measure of sampler turbulence. There is no correlation between the two quantities for either analyte. Clearly, the spectral derivatization and data collection thresholds discriminate against spectra of excessively turbulent solutions that could influence the analysis.

Total instrument uncertainties. Contributions to the total propagated instrument uncertainties come from calibration standards $\left(\sigma_{s t d}\right)$, prediction models $\left(\sigma_{c a l}\right)$, and sampler operation $\left(\sigma_{\text {sampler }}\right)$. These three factors can be reasonably considered to be independent, and thus the uncertainties are obtained by summing the squares of each of these components:

$$
\sigma_{\text {total }}^{2}=\sigma_{\text {std }}^{2}+\sigma_{\text {cal }}^{2}+\sigma_{\text {sampler }}^{2}
$$



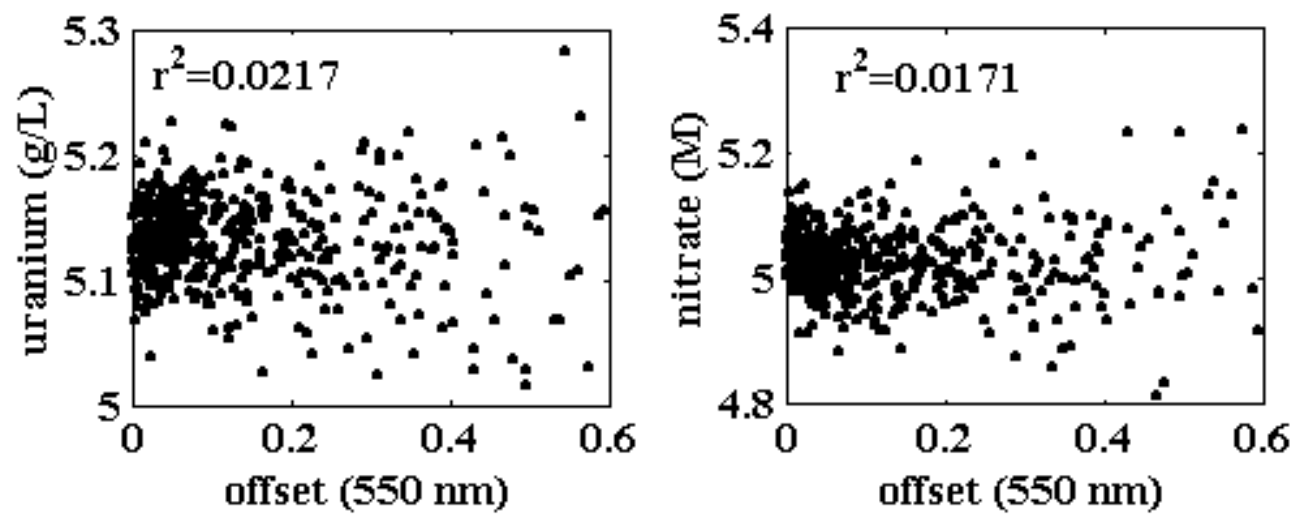

Figure 27. Influence of sampler operation on analysis accuracy.

Both the standard and model uncertainties are best expressed as percentages of the analyte, while the sampler uncertainty is assumed to be constant. Figure 28 shows the propagated uncertainties for uranium and nitrate, as a function of analyte concentration. Table 11 displays the uncertainties for each tank, based on the expected analyte concentrations listed in Table 1.

While the concentration dependences of both uranium and nitrate uncertainties both show a sharp increase at low concentrations, those increases are due to different effects. For uranium, absorbances are very low at those concentrations, and the signal-to-noise ratio is poor for the $1 \mathrm{~cm}$ optical path length used to obtain the data. None of the tanks which will be monitored in this application are expected to have such low concentrations, and thus there is no need to improve the uncertainties by using a flow cell with a longer path length. For nitrate, the uncertainty increase at low concentrations is a function of the very small changes in spectral line shape observed in that regime. As this effect depends on the chemistry of the solutions rather than a mechanical factor, an increase of the uncertainty will always be present.

The uncertainties in Table 11 imply that nitrate concentrations of $0.10 \mathrm{M}$ cannot be reliably detected. In contrast, the validation data in Table 10 show excellent agreement between this instrument and CLAB analyses. The agreement may indicate that the uncertainties are overestimated, but an explanation for why this should be the case is not immediately apparent. The primary contribution of the standard uncertainty is the uncertainty of the stock solution concentrations. The measurement error associated with that uncertainty can be determined by comparison of spectrometer results with the results of the analysis of the same solutions using a reference method. Apparent bias in the spectrometer results can be corrected, and contribution of the stock solutions to the total uncertainty can be removed. The determination of such a correction factor and the recalculation of the uncertainties are left for future work. 

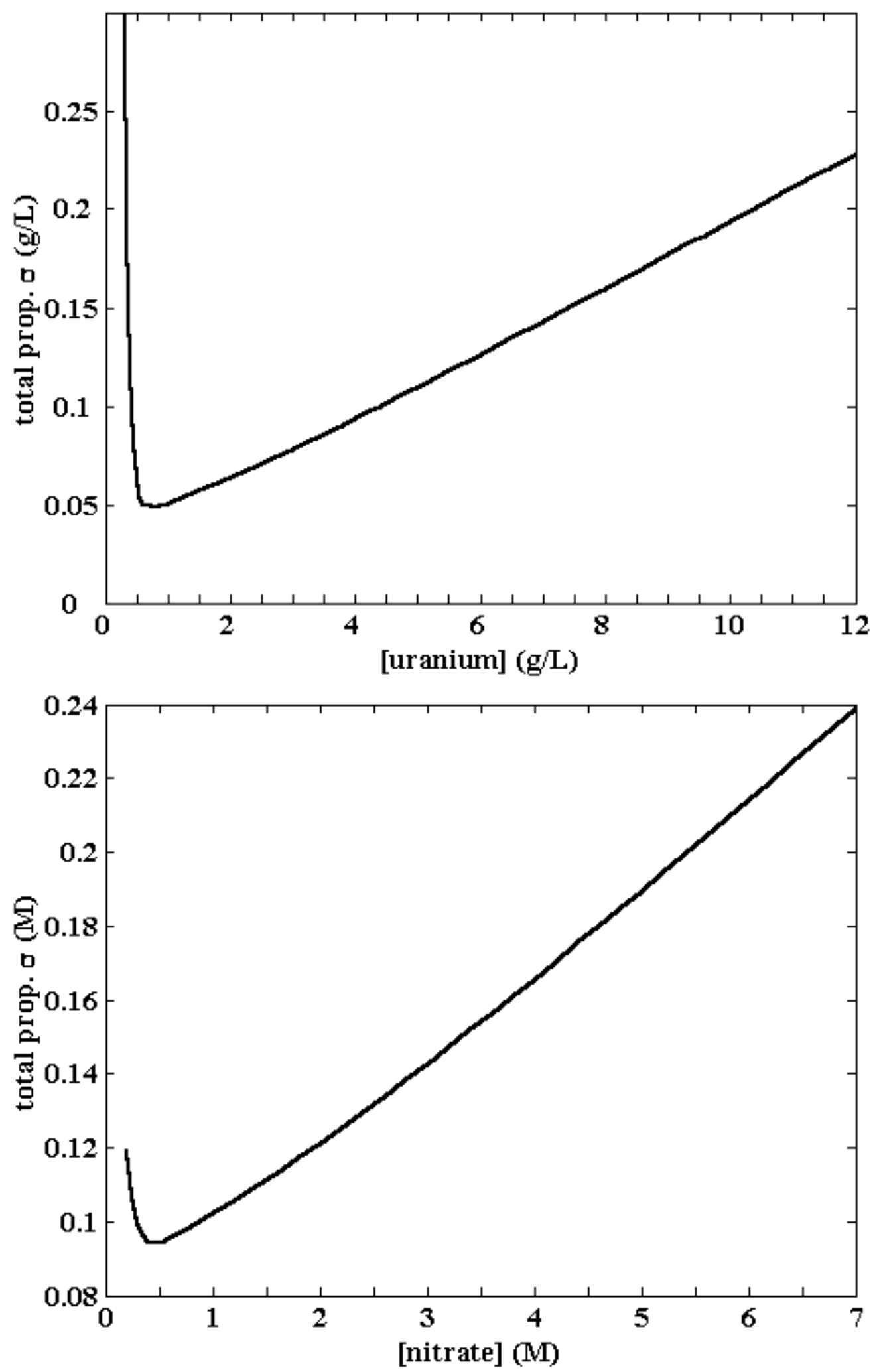

Figure 28. Total propagated uncertainties $(1 \sigma)$ for uranium and nitrate. 
Table 11. Uncertainties $(1 \sigma)$ for each measurement point.

\begin{tabular}{|c|c|c|c|}
\hline Tank & Spectrometer & Uranium $_{(\mathbf{g} / \mathbf{L})^{\mathbf{a}}}$ & ${\text { Nitrate }(\mathbf{M})^{\mathbf{a}}}$ \\
\hline 12.2 & 1 & 0.113 & 0.125 \\
\hline 12.3 & 2 & “ & “ \\
\hline $14.2^{\mathbf{b}}$ & 1 & 0.086 & 0.096 \\
\hline 14.5 & 2 & 0.156 & 0.160 \\
\hline 15.4 & 1 & “ & “ \\
\hline $15.7^{\mathbf{b}}$ & 1 & “ & “ \\
\hline 16.8 & 1 & 0.121 & 0.173 \\
\hline 17.4 & 2 & 0.156 & 0.160 \\
\hline 17.5 & 1 & 0.086 & 0.96 \\
\hline 18.1 & 2 & “ & “ \\
\hline 18.7 & 2 & 0.121 & 0.173 \\
\hline
\end{tabular}

a Where a range of concentrations has been listed in Table 1, the uncertainty reported here represents the maximum uncertainty for that range. $\mathbf{b}-$ These tanks are currently monitored with colorimeters. Their incorporation into the spectrophotometer system has been deferred.

\section{CONCLUSIONS}

The Analytical Development Section of SRTC has developed on line instrumentation for the measurement of uranium and nitrate in H Canyon in support of the HEU Blend Down project. Development work included a study of the performance characteristics of the air-lift sampler and flow cell system, specification and procurement of the two spectrophotometer systems, writing and testing of the data acquisition software, and construction of the partial least-squares chemometric models used for data analysis. The system has been demonstrated in Second Uranium Cycle for over a year and a half. New tools have been recently developed to expand the operation of the system to First Cycle Feed solutions. Those tools include new sampler/flow cell designs and prediction models incorporating potential chemical interferents. The measurement quality meets HEU project requirements. The fiber optic-based measurement system provides real-time analysis of tank contents, increasing processing rates. It also reduces radiological exposure for personnel by reducing the amount of sampling that must be carried out.

\section{ACKNOWLEDGEMENTS}

The authors thank Dr. B.B. Anderson and T.B. Edwards for technical discussions; J.C. Black, K. Palmer, J. Clark, Y. Simpkins, D. Scott, and S. Emory for experimental assistance; and B. Posnick, D. Hart, L. Bush, D. Creech, L. Chatfield, J. Dunning, Z. Madtes, C. Loyal, and D. Jones for support in H Canyon. 


\section{REFERENCES}

1) D.R. Van Hare, P.E. O’Rourke, W.S. Prather, M.B. Bowers, and M.J. Hovanec, “Online Fiber-optic Spectrophotometry”, Report DP-MS-88-186, Savannah River Laboratory, Aiken, SC (1989).

2) D.C. Harris, Quantitative Chemical Analysis, $3^{\text {rd }}$ Ed., W.H. Freeman \& Co., New York (1991).

3) S.L. Maxwell, III, "Rapid Actinide-Separation Methods", Radioactivity and Radiochem. 8, 36-44 (1997).

4) X. Wu and W. Qi, "Spectrophotometric Determination of Trace Uranium in Geological Samples by Flow-injection Analysis with Online Levextrel Resin Separation and Preconcentration.", Anal. Chim. Acta 214, 279-288 (1988).

5) M.B. Bowers, "Operational Characteristics of an Air Lift Sampling System", M.S. Thesis, Clemson University (1986).

6) R. Lascola, R.R. Livingston, M.A. Sanders, J.E. McCarty, and J.L. Dunning, "Online Spectrophotometric Measurements of Uranium and Nitrate Concentrations of Process Solutions for Savannah River Site's H-Canyon", J. Proc. Analytical Chem., 7(1), 14-20 (2002).

7) J. Bürck, "Spectrophotometric Determination of Uranium and Nitric Acid by Applying Partial Least-Squares Regression to Uranium(VI) Absorption Spectra", Anal. Chim. Acta 254, 159-165 (1991).

8) L. Couston, D. Pouyat, C. Moulin, and P. Decambox, "Speciation of Uranyl Species in Nitric Acid Medium by Time-Resolved Laser-Induced Fluorescence", Appl. Spectrosc. 49, 349-353 (1995).

9) C. Moulin, P. Decambox, P. Mauchien, D. Pouyat, and L. Couston, "Direct Uranium(VI) and Nitrate Determinations in Nuclear Reprocessing by Time-Resolved LaserInduced Fluorescence", Anal. Chem. 68, 3204-3209 (1996).

10) V.K. Bhargava, E.S. Chandrasekharan, R.H. Iyer, V.K. Rao, M.V. Ramaniah, N. Srinivasan, "In-Line Analytical Methods for Fuel Reprocessing Streams, Part I: Direct Colorimetry for Uranium and Free Acid”, Indian J. Appl. Chem. 35, $49-54$ (1972).

11) J.M. Berg, D.K. Veirs, R.B. Vaughn, M.R. Cisneros, and C.A. Smith, "Speciation Model Selection by Monte Carlo Analysis of optical Absorption Spectra: Plutonium(IV) Nitrate Complexes", Appl. Spectrosc. 54, 812-823 (2000).

12) P. Geladi, B.R. Kowalski, "Partial Least-Squares Regression: A Tutorial", Anal. Chim. Acta, 185, 1-17 (1986).

13) K.S. Booksh, "Chemometric Methods in Process Analysis", in Encyclopedia of Analytical Chemistry, R.A. Myers, Ed., 8145-8169, John Wiley \& Sons, Chichester (2000).

14) J. Workman, Jr., "The State of Multivariate Thinking for Scientists in Industry: 19802000", Chemometrics and Intelligent Lab. Sys, 60, 13-23 (2002).

15) K.R. Beebe, R.J. Pell, M.B. Seasholtz, Chemometrics: A Practical Guide, Wiley, New York (1998).

16) Y. Wang, M.J. Lysaght, and B.R. Kowalski, "Improvement of Multivariate Calibration Through Instrument Standardization”, Anal. Chem. 64, 562-564 (1992). 


\section{APPENDIX 1 - ONLINEZ.EXE DETAILS}

This appendix describes in detail the following aspects of Onlinez.exe, the primary data acquisition program: program flow, functional modules, and Visual Basic modules and forms.

\subsection{Program flow}

\section{Start Program}

Click GO button

Call StartPlot

Call FirstTime (finds highest priority position to measure)

Call ReadOptionsFile (for position to be measured.)

Call Measure (main routine in "core" program)

Call MultiMove -Tell multiplexer to move to appropriate position and get the following spectra:

1. Dark Current (Call INSTRef)

2. Reference (Call INSTRef)

3. Measurement (Call INSTMeas)

Call AnalyzeIt1 - Pass data array to model calculation routines which return concentration and goodness of fit values.

\section{Call Calculate}

Call appropriate model calculation routine (e.g. CalcPLS)

Write results to result file for position being measured.

Send results to ActiveX DLL (PlotRes.dll) for plotting, if requested.

Save spectral data if requested.

Call WriteOutputs

(if any alarm flag is set then write Digital output to FieldPoint for

DCS alarm, else write Analog output value to FieldPoint)

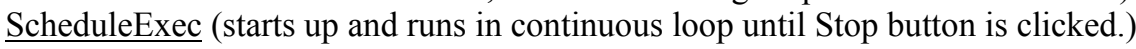

Do until Stop=True

Find highest priority position to measure for which measurement cycle time has expired.

Call ReadOptionsFile (for position to be measured.)

Call Measure (main routine in "core" program)

Call MultiMove Tell multiplexer to move to appropriate position and get the following spectra:

4. Dark Current (Call INSTRef)

5. Reference(Call INSTRef)

6. Measurement(Call INSTMeas)

Call AnalyzeIt1 - Pass data array to model calculation routines which return concentration and goodness of fit values.

Call Calculate

Call appropriate model calculation routine (e.g. CalcPLS)

Write results to result file for position being measured.

Send results to ActiveX DLL (PlotRes.dll) for plotting, if requested.

Save spectral data if requested.

Call WriteOutputs

(if any alarm flag is set then write Digital output to FieldPoint for DCS alarm, else write Analog output value to FieldPoint) 


\subsection{Functional Modules}

\section{Startup and Run Operations}

Form Online - This is the startup form for the program. It contains the controls to allow editting program parameters and to start the analytical process. This is the main control form for setting up the analysis schedule and sample point control parameters. It is used to both start the online analysis schedule and to setup and maintain that schedule. Contains the following subroutines:

Sub $M$ On

Sub SetFlexLabels

Sub O_On

Sub StartupSubOnline

\section{Core operations}

Module Mainmod - This module provides for the overall control of each individual analysis operation. It initializes and controls the discrete analyses. Contains the following subroutines:

Sub AnalyzeIt2

Sub Measure

Sub InitInfo

Sub StartUpSub

\section{Scheduling}

Module Schedmod - This module contains the routines for initializing and running the analysis schedule. It determines which analysis sample point to analyze next and then writes out any necessary results and errors/warnings to the Fieldpoint I/O modules. Contains the following subroutines:

$\begin{array}{lll}\text { Sub CheckTime } & \text { Sub LogDisplay } & \text { Sub StartPlot } \\ \text { Sub CreateLogName1 } & \text { Sub ReadSchedule } & \text { Sub WriteOuts } \\ \text { Sub FirstTime } & \text { Sub ScheduleExec } & \text { Sub WriteSchedule }\end{array}$

Sub GoMeasure Sub SetCalRanges

Form Schedule - This form allows the setup and editting of the analysis schedule by sample point including priority and time interval of each multiplexer position.

Form frmFlex - This form displays the analysis results for all positions being analyzed in the schedule.

\section{Experiment Options Setup}

Form Options - This form allows editting of the many analysis parameter associated with each individual analysis. It is used primarily in maintenance mode. Contains the following subroutines:

Sub DisableX

Sub frmInitValues

Sub OptInitValues

\section{Position Options Setup}

Form EditPos - This form allows editting of certain parameters associated with each multiplexer position. Form OptionsPos - This form allows setting and editting of the analysis parameters associated with each multiplexer position. Contains the following subroutines:

Sub frmInitValues

Sub OptInitValues

\section{Spectral Display and Control}

Form Expose - This is the spectral display screen and main control screen while the analysis schedule is executing. Contains the following subroutines:

Sub DisableX

\section{Multiplexer Control}

Module Dicon - This module contains the routines to control the Dicon multiplexer to allow sampling and analyses of multiple sample points. Contains the following subroutines:

$\begin{array}{lll}\text { Sub MultiDelay } & \text { Sub MultiMove } & \text { Sub MultiStatus } \\ \text { Sub MultiHome } & \text { Sub MultiSetup } & \text { Sub MultiTest }\end{array}$

Form Multi - This form allows manual control of the Dicon multiplexer in maintenance mode.

\section{Math Functions}

Module MathUtil - This module contains the math routines which are used for the chemometric analysis of spectral data obtained by the spectrometer. Contains the following subroutines: 
Sub AutoScale

Sub BackGround

Sub Derivative

Sub DifFunction

Sub FFT

Sub MathProcess

Form MathDialog

Form MathSelect1

Form FFTDialog
Sub MathSelect

Sub MeanCenter

Sub PeakFind1

Sub PeakFind2

Sub RemoveSpike

Sub SearchArray
Sub ShiftSpec

Sub StuffCoeff

Sub Translate

Sub VarianceScale

Model Functions

Module Qantutil - This module contains the main chemometric routines associated with the model results determination from spectral data. Contains the following subroutines:

$\begin{array}{lll}\text { Sub CalcCLS } & \text { Sub Calculate } & \text { Sub ReadMLRModel } \\ \text { Sub CalcMLR } & \text { Sub Invert } & \text { Sub ReadPCRModel } \\ \text { Sub CalcPCR } & \text { Sub LoadModel } & \text { Sub ReadPLSModel } \\ \text { Sub CalcPLS } & \text { Sub MLR } & \text { Sub ReadRegCoef }\end{array}$

Sub CalcRegCoef

Sub ReadCLSModel

Plotting Functions

Module PlotUt - This module contains the routines to enable plotting of spectral data on a display screen.

Contains the following subroutines:

Function FindPlotMax

Sub Limit

Sub PlotSetResult

Function FindPlotMaxT

Sub PlotResult

Sub SetPicHeight

Function FindPlotMin

Sub PlotSet 1

Function FindPlotMinT

Sub PlotSet2

Form ScaleIt - This form allows setting manual minimum and maximum scale values

\section{Edit Functions}

Forms Edit1, Edit2 and Edit3 - These three forms allow editting of various data in multiple column format.

\section{File Operations}

Form FileInfo - This form obtains information from the user to create a spectral saved data file.

Form SampInfo - This form obtains data regarding each analysis to be used in the saved data file.

\section{Messaging}

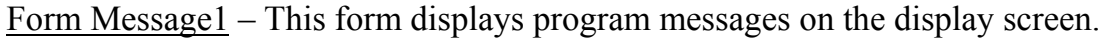

\section{Instruments Control and Interface - General}

Module InstrMod - This module contains the general purpose interface to the spectrometer for initialization, control, and data acquisition. Contains the following subroutines:
Sub CheckMultiKey
Sub GetAverageDataRef
Sub INSTRef
Sub DummyCurvel
Sub GetAverageGoodData
Sub IntensityStats
ub DummyCurve2
Sub DummyCurve3
Function GetDataIntenAve
Sub MeasLoop
Sub GetAverageData
Sub GetRefIntenAve
Sub INSTMeas

\section{Instruments Control and Interface - Specific}

Module Zss - This module contains the instrument specific code for the Zeiss Spectrometer. Contains the following subroutines:
Function GetSPdata
Sub SetGain
Sub INSTSetup
Sub RunSpecial
Sub SetupSpecial
Sub Shutter

Sub SRSErrorHandler 
Fieldpoint I/O Functions

Module FieldPoint - This module contains the routines which interface with the National Instruments

Fieldpoint input/output hardware. Contains the following subroutines:

Sub DisableFPWatchdog

Sub InitFPAnalog

Sub InitFPDigital

Sub SetupWatchdog

Sub EnableFPWatchdog

Sub PowerUpClear

Sub WriteAnaOut

Sub GetCheckSum

Sub ReadFieldPoint

Sub GetOutputChannels

Sub SetUpFieldPoint

Sub WriteDigOut

Sub InitFieldPoint

ons and Declarations

National Instrument Driver Definitions and Declar
Module Nidaq32 - Declare functions for NiDaq driver

Module Nidaqcns - Declare constants for NiDaq driver

Module Nidaqerr - Declare error constants for NiDaq driver

Module Nidex32 - Declare more constants for NiDaq driver

\section{Miscellaneous Functions}

Module Specutil - This module contains various utility routines used by other program modules.

Contains the following modules:
Sub FileExist
Sub Menuedit
Sub ReadKeyScreen
Sub ReadMvaFile
Sub Message
Sub ReadSpecSet
Sub MessageForm
Sub SetChannelFlag
Sub ParamtoSpecParam
Sub SetupMVAFile
Sub ReadHeader
Sub SetupSaveFile

Sub UpdateSpecFile

Sub WriteAsciiFile

Sub WriteHeader

Sub WriteSpecFile

Module SpecUt2 - This module contains various utility routines used by other program modules.

Sub CreateDefaultInstruData Sub ShellCopyFile

Sub

CreateDefaultOptionsFile

Sub Version

Sub WriteInstrFile

Function CreateLogName

Sub CreateModelDir

Sub WriteIntegTimeM

Function CreateMVAName

Sub WriteMessage2

Sub WriteModelNames

Sub EditInstrFile

Sub EditOptionsFile

Sub FindStartEnd

Sub FindStartEndPlot

Function FindWave

Function

FindWaveChannelNum

Sub FindWaveDummyData

Sub FullRange

Sub GetFileName

Sub GetFileNameDialog

Sub GetPosNum

Sub GetUserName

Sub GetWaveLengths

Sub GetWaveLengthsPlot

Sub GetZeroSpec

Sub InputNumScreen

Sub OptToSpecSet

Sub PeakFind1

Sub ReadInstrFile

Sub ReadIntegTimeM

Sub ReadOptionsFile

Sub ReadRefFile

Sub SetColors

Sub SetPositionOptions 
Form ChPath

Form GetNum

Form frmFlex

Form frmStop

Form ReadKey1

Form SelDir

Form XUnit

Empty Routines

Module Dummy2 


\subsection{Visual Basic Modules and Forms contained in Onlinez program}

\section{Form Name}

\author{
Form $=.$. OOPTIONS.FRM \\ Form $=.$. BLANK.FRM \\ Form $=.$. EDIT1.FRM \\ Form $=.$. EDIT2.FRM \\ Form $=.$. EDIT3.FRM \\ Form $=.$. IGETNUM.FRM \\ Form=..IMATHDIA.FRM \\ Form $=.$. MATHSEL.FRM \\ Form $=.$. READKEY1.FRM \\ Form=.. XXUNIT.FRM \\ Form $=.$. MOPTIONS.FRM \\ Form $=$. . IOPTPI.FRM \\ Form $=$. ..FFTDIA.FRM \\ Form $=.$. REGIONSE.FRM \\ Form $=.$. MUULTI.FRM \\ Form $=.$. ISCALEIT.FRM \\ Form $=$.. $\mid$ CHPATH.FRM \\ Form $=.$. IFILEINFO.FRM \\ Form $=.$. ISAMPINFO.FRM \\ Form=... SelDir.frm \\ Form=.. loptionsP.frm \\ Form $=.$. Expose 33.frm \\ Form $=.$. IOnline.frm \\ Form=... Schedule.frm \\ Form=..|EditPos.frm \\ Form $=$. .. Message. .rm \\ Form=...Message2.frm
}

\section{Module Name}

Module=DUMMY2; ..IDUMMY2.BAS

Module=MAINMOD; ...MAINMOD.BAS

Module=MATHUTIL; ...MATHUTIL.BAS

Module=PLOTUT; ...PLOTUT.BAS

Module $=$ Qantutil; ... IQANTUTIL.BAS

Module=SPECUTIL; .. ISPECUTIL.BAS

Module $=$ SpecUt2; .. Ispecut 2 .bas

Module=Instrmod; .. Instrmod.bas

Module $=$ Nidaq $32 ;.$. Nidaq32.bas

Module $=$ Nidex $32 ;.$. Nidex32.bas

Module $=$ Nidaqcns; ... Nidaqcns.inc

Module $=$ Nidaqerr; ... Nidaqerr.inc

Module $=$ Schedmod; .. ISched.bas

Module $=$ dicon; ... dicon.bas

Module=FieldPoint; ...FPmod.bas

Module $=$ zss; ZS.bas

\section{Description}

Used to display, edit and save options.dat data

Blank screen - Not currently used

Generic data field edit screen - 1 column

Generic data field edit screen - 2 columns

Generic data field edit screen - 3 columns

Used to get a numeric value from user

Used to obtain info for derivative function

Used to select a math function

Used to obtain an ASCII key from user

Not currently used

Not currently used

Not currently used

Used to obtain info for FFT math function

Not currently used

Used for manual control of multiplexer

Used to obtain min and max scale values

Used to change the current file path

Used to obtain info for creating MVA data file

Used to obtain info about sample for MVA file

Used to select a file directory

Used to display, edit and save options?.dat data

Screen to graphically display spectral data

Main screen for program (start-up form)

Used to display, edit and save schedule

Edit screen to edit additional position options

Displays program messages on expose form

Displays program messages on message form

\section{Description}

Dummy routines

Main module of "core program"

Math Routines

Plotting Routines

Model calculation Routines

Misc. Utilities

More Misc. Utilities

Spectrometer control routines

Nidaq declarations

Nidaq constant definitions

More Nidaq constant definitions

Nidaq error constant definitions

Main routines of "shell program"

Dicon multiplexer routines

FieldPoint I/O routines

Zeiss spectrometer routines 


\section{APPENDIX 2 - SAMPLE UNCERTAINTY CALCULATION}

The uranium concentrations and uncertainties of the calibration and validation solutions are calculated from the masses $(m)$ and densities $(d)$ of the aliquots of the uranium and nitrate stock solutions (US and NS, respectively), as follows.

$$
[U]_{\text {final }}=[U]_{U S} \times \frac{m_{U S}}{d_{U S}} \times \frac{d_{\text {final }}}{\sum m_{U S+\text { water }+A S}},
$$

and

$$
\left(\frac{\sigma_{[U]_{\text {final }}}}{[U]_{\text {final }}}\right)^{2}=\left(\frac{\sigma[U]_{U S}}{[U]_{U S}}\right)^{2}+\left(\frac{\sigma_{m_{U S}}}{m_{U S}}\right)^{2}+\left(\frac{\sigma_{d_{U S}}}{d_{U S}}\right)^{2}+\left(\frac{\sigma_{d_{\text {final }}}}{d_{\text {final }}}\right)^{2}+\left(\frac{\sqrt{\sum \sigma_{m_{U S+\text { water }+A S}}^{2}}}{\sum m_{U S+\text { water }+ \text { AS }}}\right)^{2} .
$$

The nitrate concentrations and uncertainties of the calibration and validation solutions are calculated as follows.

$$
[N]_{\text {final }}=\left(\left[[N]_{U S} \times \frac{m_{U S}}{d_{U S}}\right]+\left[[N]_{A S} \times \frac{m_{A S}}{d_{A S}}\right]\right) \times \frac{d_{\text {final }}}{\sum m_{U S+\text { water }+A S}}
$$

and

$$
\left(\frac{\sigma_{[N]_{\text {final }}}}{[N]_{\text {final }}}\right)^{2}=\left(\frac{\sigma^{2} T_{U S}+\sigma_{T_{N S}}^{2}}{T_{U S}^{2}+T_{N S}^{2}}\right)+\left(\frac{\sigma_{d_{\text {final }}}}{d_{\text {final }}}\right)^{2}+\left(\frac{\sqrt{\sum \sigma_{m_{U S+\text { water }+A S}}^{2}}}{\sum m_{U S+\text { water }+A S}}\right)^{2}
$$

where

$$
T_{U S}=[N]_{U S} \times \frac{m_{U S}}{d_{U S}} ;\left(\frac{\sigma_{T_{U S}}}{T_{U S}}\right)^{2}=\left(\frac{\sigma_{[N]_{U S}}}{[N]_{U S}}\right)^{2}+\left(\frac{\sigma_{m_{U S}}}{m_{U S}}\right)^{2}+\left(\frac{\sigma_{d_{U S}}}{d_{U S}}\right)^{2}
$$

and

$$
T_{N S}=[N]_{N S} \times \frac{m_{N S}}{d_{N S}} ;\left(\frac{\sigma_{T_{N S}}}{T_{N S}}\right)^{2}=\left(\frac{\sigma_{[N]_{N S}}}{[N]_{N S}}\right)^{2}+\left(\frac{\sigma_{m_{N S}}}{m_{N S}}\right)^{2}+\left(\frac{\sigma_{d_{N S}}}{d_{N S}}\right)^{2}
$$

Example calculations for a solution with nominal concentrations of $2.15 \mathrm{~g} / \mathrm{L} \mathrm{U}$ and $1.0 \mathrm{M}$ nitrate are presented below. Uncertainties are as discussed in the text.

$$
[U]_{\text {final }}=33.38 \mathrm{~g} / \mathrm{L} \times \frac{1.5070 \mathrm{~g}}{1.0442^{\mathrm{g} / \mathrm{mL}}} \times \frac{1.0338 \mathrm{~g} / \mathrm{mL}}{(1.5070+18.2786+3.3931) \mathrm{g}}=2.15 \mathrm{~g} / \mathrm{L}
$$




$$
\begin{aligned}
& \sigma_{[U]_{\text {final }}}=2.15 \mathrm{~g} / \mathrm{L} \times\left(\left(\frac{0.39 g / L}{33.38^{g} / L}\right)^{2}+\left(\frac{0.0015 g}{1.5070 g}\right)^{2}+\left(\frac{0.001 \mathrm{~g} / L}{1.0442^{g} / L}\right)^{2}+\left(\frac{0.001 \mathrm{~g} / L}{1.0338^{g} / L}\right)^{2}+\left(\frac{0.1858 g}{23.1787 g}\right)^{2}\right)^{\frac{1}{2}} \\
& =0.03 \mathrm{~g} / \mathrm{L} \\
& {[N]_{\text {final }}=\left(\left[0.3282 M \times \frac{1.5070 \mathrm{~g}}{1.0442 \mathrm{~g} / \mathrm{mL}}\right]+\left[8.11 \mathrm{M} \times \frac{3.3931 \mathrm{~g}}{1.2504 \mathrm{~g} / \mathrm{mL}}\right]\right) \times \cdots} \\
& \frac{1.0338^{g} / \mathrm{mL}}{(1.5070+18.2786+3.3931) g}=1.02 \mathrm{M} \\
& \sigma_{T_{U S}}^{2}=(0.474 M \cdot m L)^{2} \times\left(\left(\frac{0.0066 M}{0.3282 M}\right)^{2}+\left(\frac{0.0015 g}{1.5070 g}\right)^{2}+\left(\frac{0.001 \mathrm{~g} / m L}{1.0442 \mathrm{~g} / m L}\right)^{2}\right)=9.02 \times 10^{-5}(M \cdot m L)^{2} \\
& \sigma_{T_{N S}}^{2}=(22.01 M \cdot m L)^{2} \times\left(\left(\frac{0.162 M}{8.11 M}\right)^{2}+\left(\frac{0.0034 g}{3.3931 g}\right)^{2}+\left(\frac{0.001 \mathrm{~g} / m L}{1.2504 \mathrm{~g} / m L}\right)^{2}\right)=0.1945(M \cdot m L)^{2} \\
& \sigma_{[N]_{\text {final }}}=1.02 M \times\left(\left(\frac{9.02 \times 10^{-5}+0.1945(M \cdot m L)^{2}}{0.474^{2}+22.01^{2}(M \cdot m L)^{2}}\right)+\left(\frac{0.001 \frac{g}{L}}{1.0338^{g} / L}\right)^{2}+\left(\frac{0.1858 g}{23.1787 g}\right)^{2}\right)^{\frac{1}{2}} \\
& =0.021 \mathrm{M}
\end{aligned}
$$

